\title{
A Model of the Equatorial Deep Jets and the Role of the Horizontal Coriolis Parameter \\ by
}

Xiaoming Zhang

B.S., Zhejiang University (1982)

M.S., The University of Michigan (1989)

SUBMMITED IN PARTIAL FULFILLMENT OF THE REQUIREMENTS FOR THE DEGREE OF

MASTER OF SCIENCE

at the

\section{MASSACHUSETTS INSTITUTE OF TECHNOLOGY \\ and the}

\section{WOODS HOLE OCEANOGRAPHIC INSTITUTION}

January, 1991

This thesis is not subject to U.S. copyright.

Signature of the author

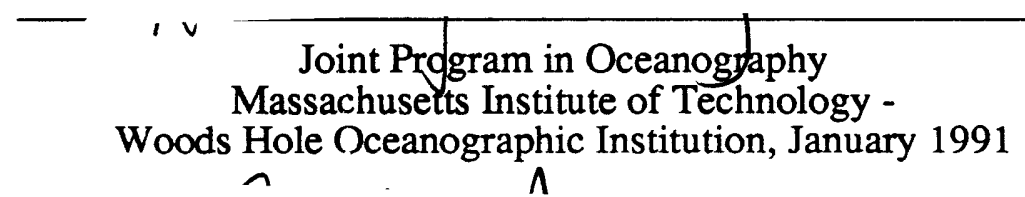

Certified by

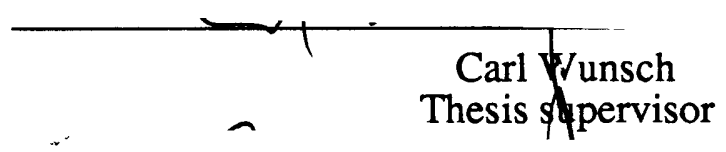

Accepted by

Carl Wunsch

Chairman, Joint Committee for Physical Oceanography, Massachusetts Institute of Technology massachUterrestudidne Oceanographic Institution OF TECHNOI OGY

MAY 141991

.. LHBARIES

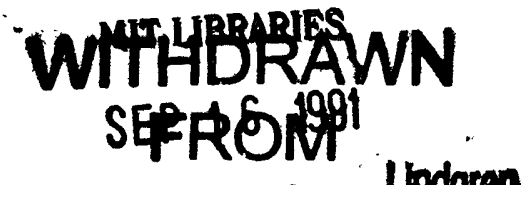




\title{
A MODEL OF EQUATORIAL DEEP JETS AND THE ROLE OF THE HORIZONTAL CORIOLIS PARAMETER
}

\author{
by \\ Xiaoming Zhang \\ Submitted to the Massachusetts Institute of Technology - \\ Woods Hole Oceanographic Institution Joint Program \\ in January, 1991 in partial fulfillment of the \\ requirements for the Degree of Master of Science
}

\begin{abstract}
This thesis reviews observational and theoretical work on the equatorial deep jets and work related to the study of the role of the horizontal Coriolis parameter. Most existing analytical models interpret the equatorial deep jets as either low frequency, long Rossby waves or stationary, long Kelvin waves generated at or near the ocean surface. These models are unable to answer the question of how wind generated energy propagates down through the equatorial undercurrent and thermocline into the deep ocean. Existing numerical models do not display deep jet features due mainly to their in low vertical resolution and the high eddy viscosity associated with these models. These numerical models also suggest that very little energy is able to get into the deep ocean. A natural question is raised: can the equatorial deep jets possibly be interpreted as free, steady inertial motion below the thermocline?
\end{abstract}

We develop a simple model for the deep jets as a free, stationary inertial motion. After scaling the fluid dynamical equations in the appropriate regime, it is found that neither the advective nonlinearity nor the horizontal Coriolis parameter can be neglected. An important conservation equation, the so called potential zonal vorticity conservation equation which governs the equatorial steady and zonal independent equatorial flow is derived. From this conservation principle, an inertial equatorial deep jets model is developed which captures some important features of the deep jets. The horizontal Coriolis parameter is important in this inertial model. 
The role of the horizontal Coriolis parameter has long been controversial in the literature. We discuss this role for several equatorial flow systems. It is found that the horizontal Coriolis parameter is not significant for inviscid linear equatorial waves due to the presence of stratification in the real ocean. However, when the ratio of momentum eddy viscosity to the density dissipation coefficient becomes small enough, the effect of the horizontal Coriolis parameter becomes more important in a simple viscous model. Some general aspects of this parameter have also been discussed in terms of angular momentum conservation and energy conservation principles. It is suggested that for the ocean circulation of large vertical excursion of the fluid particle, the horizontal Coriolis parameter effect may not be small and should be included in future numerical models.

Thesis Supervisor: Carl Wunsch

Cecil and Ida Green Professor of Physical Oceanography

Department of Earth, Atmospheric and Planetary Sciences

Massachusetts Institute of Technology 


\section{Table of Contents}

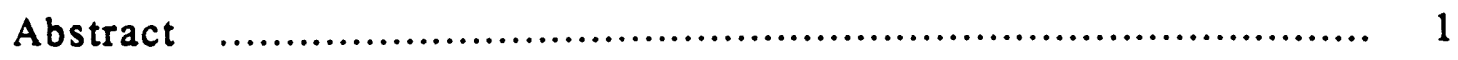

1. Review of observations and theories of equatorial deep jets ....................... 4

2. Review of the traditional approximation ................................................ 8

3. Scaling and simplification of equations in equatorial deep jets regime ............ 14

4. General discussion on the horizontal Coriolis parameter ............................ 19

5. Potential zonal vorticity conservation ............................................... 24

6. An inertial equatorial deep jets model ............................................... 28

7. Role of the horizontal Coriolis parameter in linear equatorial waves ............... 39

8. Effect of momentum friction and density diffusion .................................. 49

9. Concluding Remarks …........................................................ 51

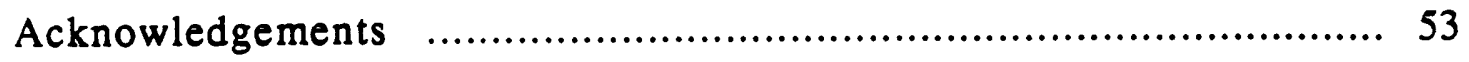

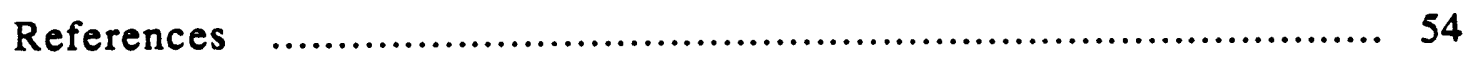




\section{Section 1. Review of observations and theories of equatorial deep jets}

\subsection{Observations}

Observation reveals an important phenomenon in equatorial oceans: the presence of strong, alternating zonal currents with small vertical scale trapped near the equator. After their first discovery by Luyten and Swallow (1976) in the western Indian Ocean, these energetic flows called the equatorial deep jets (EDJ) were also observed in the Equatorial Pacific (Hayes and Milburn, 1980; Leetmaa and Spain, 1981; Eriksen, 1981, Firing, 1987, Ponte and Luyten, 1989) and in the Atlantic Ocean (Eriksen, 1982, Ponte, Luyten and Richardson, 1989). A typical zonal velocity contour in the meridional plane obtained by Firing(1987) is shown in Fig.1.1. The right panel shows that below the equatorial undercurrent (with meridional extension 2-3 degrees north and south away from equator), there are eastward and westward alternating jets starting from the westward equatorial intermediate current down to about $2500 \mathrm{~m}$. The zonal velocity magnitude becomes smaller for deeper jets. Although the left panel shows that the standard deviation of the measurement is of the same order as the signal of the deep jets, this phenomenon is generally believed to be a real feature, because it has been also observed in many other locations. Fig.1.2, Fig.1.3, Fig.1.4 and Fig.1.5 are from Ponte and Luyten (1989), Ponte(1988a) which show the zonal and meridional distributions of zonal velocity and meridional velocity in the central Pacific equatorial ocean. Fig.1.6 is a vertical wavenumber spectrum (Ponte and Luyten, 1989) and clearly shows within the $95 \%$ confidence level that the $O(100 \mathrm{~m})$ jets signals are dominant. 
Some important features are summarized as follows which are generally true for most equatorial deep jets observed in different geographical parts of equatorial oceans. They are:

(1) Alternating zonal currents of velocity order $\mathrm{O}(10 \mathrm{~cm} / \mathrm{sec})$ sit on the equator trapped to within 1 degree north and south latitude from the equator. (The meridional trapping length scale is of order $\mathrm{O}(100 \mathrm{~km}))$;

(2) Very long zonal length scale of order longer than $10,000 \mathrm{~km}$.

(3) Very small vertical scale approximately $\mathrm{O}(100 \mathrm{~m})$, extend to the deep ocean, although the signal becomes obscure in oceans deeper than $\mathrm{O}(3000 \mathrm{~m})$.

(4) Large time scale of order more than 4 years (Ponte and Luyten, 1989). The motion is not distinguishable from stationary (Firing, 1987) because the longest measurement record for detecting equatorial deep jets is no longer than 4 years.

(5) Reasonable agreement found between direct zonal current measurements and geostrophic velocities calculation from observed density field (Eriksen, 1982).

\subsection{Theories}

The interpretation of the jets as low-frequency surface forced, vertically propagating linear equatorial waves has been advocated in the past. The first model was given by Wunsch (1977). His model jets consist of long Rossby waves forced at the annual period by a surface vertical velocity pattern of a particular zonal wavenumber and are unbounded in the zonal direction. McCreary and Lukas (1986) hypothesized that the deep equatorial jets might be near-resonantly excited, wind-forced, stationary Kelvin waves embedded in a barotropic mean westward flow. Ponte (1988b) examined the effects of slowly varying baroclinic westward flow on stationary Kelvin waves.

There are some difficulties associated with the current existing models. Ponte and Luyten(1989) stated that interpretation of signals in terms of equatorial waves was 
ambiguous, because of their relatively long spatial and temporal scales compared to that of the record. They found that it was hard to fit the meridional structure of the model wave to observations. The energy level decays too fast in the meridional direction away from the equator in the wave model. The simplest hypothesis of linear waves in a resting basic state ocean could not be rejected, but more complicated physics cannot be ruled out. There are at least three major difficulties associated with the surface generated, downward propagating wave models although they are still controversial. (1) Gent and Luyten (1985) claimed that the existence of a strong thermocline peak in the Brunt Väisälä frequency profile might reflect most of the wave energy, preventing it from to penetrating through. (2) McPhaden et al. (1986) suggested that the critical levels at depth were a significant barrier to vertical energy propagation. Energy at short $\mathrm{O}(100 \mathrm{~m})$ vertical scales would be blocked from entering the deep equatorial ocean by the existence of the shallow critical levels in the eastward Equatorial Undercurrent and westward South Equatorial Current system. (3) McCreary (1984) pointed that for reasonably long time scales, equatorial waves propagated energy into the deep ocean at very shallow angles to the surface because the vertical group velocity was very small - This assumes that the argument that energy propagates at the speed of group velocity in such a varying background medium can still be used. The turbulent diffusion may quickly dissipate most of the small vertical scale wave energy before it could get very deep. All of these three difficulties proposed one question: how does the wave energy get into the deep ocean? These difficulties have not been totally resolved. Furthermore, the stationary models by McCreary and Lukas (1986), Ponte (1988b) that relate the deep equatorial jets to stationary forced Kelvin waves rely on an assumed deep westward mean flow that is poorly documented in observations. So the above wave models are still questionable.

To avoid these difficulties, Ponte (1988b) suggested that some of the deep jets might be forced by variations in the mean vertical-velocity field of the Equatorial Undercurrent itself. Ponte (1990) also studied the deep lateral boundary processes (e.g. 
western boundary currents) as an alternative energy source exciting the equatorial wave guide at long time scales. However, the question of the origin of this forcing was not examined.

More sophisticated numerical models have also been developed in the literature (Philander and Pacanowski, 1981, 1984, Rothstein, et al., 1985, 1988) to simulate the equatorial dynamics. . However, up to now no numerical model has successfully modeled the equatorial deep jets. The major reasons may be of two types: (1) Nonlinear numerical models need a large eddy viscosity to make the numerical calculation stable, and this large unrealistic value of eddy viscosity may well dissipate the small scale energy associated with the interesting features. (2) The current numerical models have too low vertical resolution. Most models have fewer than 17 levels in the vertical and most of these levels are concentrated in the upper ocean, trying to resolve the equatorial undercurrent. Thus, understanding the dynamics of the deep jets remains an interesting problem. Therefore it is important, if possible, to analyze the nonlinear dynamical system of the deep equatorial ocean, even if only qualitatively.

Since the surface generated, or near surface generated energy is unlikely to penetrate into deep ocean and since that the equatorial deep jets are in the nonlinear dynamics regime (See Section 3), it is natural for us to ask a question: Can the equatorial deep jets be interpreted as free, steady inertial motion? Although the above mentioned numerical models addressed the nonlinearity, the low resolution and large eddy viscosity prevented them from being able to answer this question. As a preliminary analysis, in this thesis, we propose a simple model trying to answer this question qualitatively. The conclusion is that it is possible. 


\section{Section 2. Review of the traditional approximation}

The traditional approximation is widely used in geophysical fluid dynamics. The main idea is that in a shallow ocean $(\delta=H / a<<1, H$ is the depth of ocean, a is the radius of earth), the Coriolis acceleration associated with the horizontal component of the earth's rotation and the vertical component of the fluid particle acceleration can be neglected (Eckart, 1960). The significance of this approximation is that it greatly simplifies the mathematical complexities of the physical problem and also emphasizes some important physical features of interest.

After using this approximation, the vertical momentum equation becomes the hydrostatic relation so that it is possible to obtain spatially separable solutions for linear

systems under favorable boundary conditions. Thus the traditional approximation not only makes the analytical discussion of problems easier, but it also makes numerical computation more efficient by using vertical modes or layer models. The validity of this approximation has long been controversial (see Bjerknes et al. 1933, Proudman 1942, Phillips 1966, 1968, Veronis 1968, Miles 1974, and Hendershott 1981). A definite conclusion is still lacking. This thesis is not intended to give a universal criterion for the validity of the traditional approximation. Rather, it studies some possible roles of the Coriolis acceleration associated with the horizontal component of the earth's rotation in some different physical situations, especially the equatorial dynamical systems. A short review of the historical controversies and several models without the traditional approximation is given in this section.

The traditional approximation is composed of three sub-approximations: 1) Neglect of the radial variation of the metrical coefficients $(r \approx a)$. It has been shown (Miles, 1974) that the errors are uniform in $O(\delta)$ and are no more significant than those implied by the assumption of a spherical planet with a uniform gravitational field. 
2) Neglect of the Coriolis acceleration associated with the horizontal component of earth's rotation, $2 \Omega \cos \varphi w,-2 \Omega \cos \varphi$ in zonal and vertical momentum equations, respectively, where $\Omega$ is the earth's rotational angular velocity.

3) Neglect of the vertical acceleration $\partial w / \partial t$.

Miles (1974) showed that for $N \gg>2 \Omega$, where $N$ is the Brunt Väisälä frequency which characterizes the vertical static stability, approximation 2 dominates approximation 3. So a large part of the controversy concerns the validity of neglecting the effect of the horizontal Coriolis parameter".

It has been recognized since the work of Bjerknes, Bjerknes, Solberg and Bergeron (1933) that the above assumptions especially the neglecting of horizontal Coriolis parameter amounts to more than a minor perturbation of the spectrum of free oscillations that may occur in a thin homogeneous ocean. They realized that the hydrostatic assumption excluded a class of free inertial (of period longer than one pendulum day) oscillations of the homogeneous fluid.

Solberg (1928) showed that the assumption had little effect for the wave motion of period shorter than one day. Proudman (1942) argued that the large aspect ratio $\delta$ effectively eliminated these oscillations as major components of the astronomically forced tide except possibly in the case of inertial oscillations near the poles and near the equator.

Stewartson and Rickard (1969) pointed out that the limiting case of a vanishingly thin homogeneous ocean is a nonuniform limit for motion $\sigma<2 \Omega$ (period longer than one pendulum day, $\sigma$ is the temporal frequency of the wave motion). The solution obtained by solving the equations and then taking the limit may be different from those obtained by first taking the limit and then solving the resulting approximated equations. For motion $\sigma 2 \Omega$,

\footnotetext{
* The traditional approximation excludes a zonal Coriolis acceleration component term and a vertica: Coriolis acceleration component term in zonal and vertical momentum equations respectively. The usually used name "horizontal Coriolis component" is not very proper. We will use "horizontal Coriolis parameter" to refer to both Coriolis acceleration component terms which associated with the horizontal component of earth's rotation.
} 
no such difficulty appears to arise even for a rotating stratified fluid if $N \gg>2 \Omega$, the expansion in $\delta$ is regular. However, the difficulty does arise if $N \gg 2 \Omega$ and $\sigma<2 \Omega$ and stems essentially from Coriolis accelerations coupling between barotropic and baroclinic wave motions. The equatorial deep jets appear to be in this difficult range.

Phillips (1968), after a controversy with Veronis (1968), argued that it was the realistically large stratification that saved the traditional approximation to the full linearized equations when the ocean is very thin. However, his argument was in a WKBJ sense which assumed that all the coefficients in the linearized equations were constants. Furthermore, it was assumed in his analysis that waves were sinusoidal in all three dimensions. The discussion was only made to the effect of the horizontal Coriolis parameter on the dispersion relation. We know that the equatorial waves have a special property that their meridional structures are trapped near the equator. The wave structure in the meridional direction is in a guided wave form which is not sinusoidal. The vertical Coriolis parameter approaches to zero and the horizontal Coriolis parameter reaches its maximum at the equator. The assumption of constant coefficients in the equations is questionable since the $\beta$-effect is essential to the waves. Then what is the effect of the horizontal Coriolis parameter on equatorial waves, on the dispersion relation and also on the wave spatial structure? What is the dynamical role of the horizontal Coriolis parameter in general equatorial dynamics? Will the inclusion of other physical factors like nonlinearity, momentum diffusion and density diffusion make things different? These questions are still unanswered. Therefore, Phillips' argument is not conclusive and more analysis is needed.

In fact, some theoretical analyses have been proposed in literature and some of the results have suggested that the effect of the horizontal Coriolis parameter is significant. It was first explicitly incorporated into the limit process producing the Laplace Tidal Equations by Miles (1974) who addressed all assumptions by defining appropriately small parameters and examining the properties of expansion in them. He found that the boundary 
value problem for free oscillations of angular frequency $\sigma$ was not well posed if $\sigma^{2}<N^{2}+4 \Omega^{2}$, and that the governing partial differential equation is elliptic/hyperbolic on the polar/equatorial sides of the inertial latitudes given by $\pm \sigma=2 \Omega \sin \varphi$ if $\sigma<2 \Omega<<\mathrm{N}$. The coupling between barotropic and baroclinic modes is uniform in $O(\delta)$ if $\sigma>2 \Omega$, but it induces significant currents and vertical internal displacements between the inertial latitudes if $\sigma<2 \Omega<<\mathrm{N}$. This internal displacement could dominate those of the basic motion, but the free surface displacement remains as in the traditional approximation. These two types of motion are distinct in the limit $\delta \rightarrow 0$ and permit regular perturbation expansions in $\delta$ if and only if $\sigma>2 \Omega$; they are coupled for $\delta>0$, and lead to singular perturbation expansions, if $\sigma<2 \Omega$ (Miles 1974). Low frequency equatorial waves are in the range of $\sigma<2 \Omega<<\mathrm{N}$. It is of interest to investigate this problem in more detail. Section 7 of this thesis is devoted to this study.

Dobryshman (1980) studied extensively the equatorial atmospheric dynamics with special consideration given to the horizontal Coriolis parameter. He always incorporated the horizontal Coriolis parameter in his wave model (1987) and stationary meridional plane modon model (1988). It was claimed that the parameter was very important. However, in most of his studies, he always assumed that the pressure gradient was of a special form claimed to be consistent with meteorological observations. In the oceanographic context, the pressure gradient distribution associated with the oceanic motion signal in the equatorial deep ocean is not clear at all. Pressure perturbations need to be computed from the perturbed motion and are essentially an unknown variable. Therefore, in all of our analysis, the pressure gradient is only obtained after the final equations have been solved.

Munk and Phillips (1968) showed that the neglected Coriolis terms in their study were proportional to $\mathrm{m}^{1 / 3}$ ( $\mathrm{m}$ is vertical mode number) for internal modes and the traditional approximation may be untenable for small vertical scale internal waves. This result suggested that for small vertical scale internal waves, the horizontal Coriolis parameter might be significant. 
Models which include the horizontal Coriolis parameter for linear homogeneous equatorial ocean systems have been studied in fair detail. Stern (1963), Aldridge(1967) and Israeli (1972) found axisymmetric equatorially trapped normal modes in a rotating spherical shell of homogeneous fluid that were extinguished by the hydrostatic approximation in analytical, experimental and numerical approaches respectively. Bretherton (1964) explained these modes as due to continued reflection of low frequency inertial waves whose group velocity made a small angle with the axis of rotation between the boundaries.

Grimshaw (1975) studied the propagation of internal gravity waves in a vertically sheared flow considering the presence of the horizontal Coriolis parameter. He showed that it was possible for internal gravity waves to transmit energy through critical levels if certain relations were satisfied.

Joyce (1988) computed the zonal velocity correction due to the vertical component of the Coriolis acceleration to the usual calculation of geostrophic currents near the equator. He found that the correction was significant and reached a peak speed of $10 \mathrm{~cm} / \mathrm{s}$ close to the ocean surface.

The inaccuracies in measuring vertical velocity and pressure anomaly prevent us from being able to have a direct estimate of the importance of the horizontal Coriolis parameter from real field observations. It seems that this parameter plays a significant but not dominant role in many analyses. Its dynamic role is still unclear.

This thesis is mainly in two parts. One develops a simple inertial model for the equatorial deep jets and the other is a systematic examination of the role of the horizontal Coriolis parameter in several equatorial dynamical models.

We carefully scale and simplify the governing equations in the equatorial deep jets regime in Section 3 and find that neither the effects of horizontal Coriolis parameter not the advective nonlinearity can be ignored. After a general discussion of the horizontal Coriolis parameter in Section 4 we derive a temporary so called potential zonal vorticity 
conservation equation in Section 5. A simple nonlinear inertial model is developed in Section 6 which is capable of capturing some important features of equatorial deep jets. The $\beta$-effect due to the earth's sphericity confines the jets to the equator. The effect of density anomaly sets their vertical structure. In Section 7, we study the effect of the horizontal Coriolis parameter in equatorial linear inviscid waves. It is found that the effect on the dispersion relation and the velocity structures is not significant because of the presence of the realistically strong stratification, while the effect on the perturbed hydrostatic relation is significant within some limited regions. After the inclusion of momentum friction and density diffusion in Section 8 , it is found that the importance of the effect depends on a nondimensional parameter, $\gamma^{2}=\frac{N^{2} A_{v}}{f_{0}^{2} K_{v}}$ where $f_{0}=2 \Omega$ is the value of the horizontal Coriolis parameter at the equator, $A_{V}$ is the vertical eddy viscosity for the momentum and $\mathrm{K}_{\mathrm{V}}$ is the dissipation coefficient in the density equation. In the nonlinear equatorial deep jets model, the effect of horizontal Coriolis parameter is significant. 


\section{Section 3. Scaling and Simplification of Equations in Equatorial Deep Jets Regime}

We start from an equation set uniformly valid in Cartesian coordinate (Miles, 1974).

$$
\begin{gathered}
\frac{\partial u}{\partial t}+u \frac{\partial u}{\partial x}+v \frac{\partial u}{\partial y}+w \frac{\partial u}{\partial z}-2 \Omega \sin \varphi v+2 \Omega \cos \varphi w \\
=-\frac{1}{\rho_{0}} \frac{\partial P}{\partial x}+A_{v} \frac{\partial^{2} u}{\partial z^{2}} \\
\begin{array}{c}
\frac{\partial v}{\partial t}+u \frac{\partial v}{\partial x}+v \frac{\partial v}{\partial y}+w \frac{\partial v}{\partial z}+2 \Omega \sin \varphi u \\
=-\frac{1}{\rho_{0}} \frac{\partial P}{\partial y}+A_{v} \frac{\partial^{2} v}{\partial z^{2}} \\
\frac{\partial w}{\partial t}+u \frac{\partial w}{\partial x}+v \frac{\partial w}{\partial y}+w \frac{\partial w}{\partial z}-2 \Omega \cos \varphi u \\
=-\frac{1}{\rho_{0}} \frac{\partial P}{\partial z}-\frac{\rho}{\rho_{0}} g+A_{v} \frac{\partial^{2} w}{\partial z^{2}} \\
\frac{\partial u}{\partial x}+\frac{\partial v}{\partial y}+\frac{\partial w}{\partial z}=0 \\
\frac{\partial \rho}{\partial t}+u \frac{\partial \rho}{\partial x}+v \frac{\partial \rho}{\partial y}+w \frac{\partial \rho}{\partial z}+w \frac{\partial \rho_{0}}{\partial z}=K_{v} \frac{\partial^{2} \rho}{\partial z^{2}}
\end{array}
\end{gathered}
$$

where the Boussinesq approximation has been used and the basic hydrostatic part of the pressure has been removed. For simplicity, the horizontal diffusion terms have been neglected. It should be noticed that the acceleration terms associated with the horizontal Coriolis parameter are included in (3.1.a) and (3.1.c). Eddy viscosity and density diffusivity are $A_{v}$ and $K_{v}$, respectively, and will be assumed constant, as will be the background vertical density gradient. Other notation in the above equations is standard.

Although it is observed in the equatorial ocean that a mean meridional density gradient associated with geostrophic zonal velocity exists (Joyce, 1988), as a first approxirration, we neglect this effect since this mean meridional density gradient is very small and can hardly be distinguished from the perturbations. 
We scale the above equations by the following characteristic scales associated with the observed equatorial deep jets. Noticing that the vertical Coriolis parameter vanishes on the equator and gets larger away from the equator, the scaling is not completely uniform.

The following scales are chosen to characterize the equatorial deep jets.

$\mathrm{t}: \mathrm{T} \geq 4$ years $=\mathrm{O}\left(1.3 \times 10^{8} \mathrm{sec}\right)$

$x: L_{x}=10,000 \mathrm{~km}=O(109 \mathrm{~cm})$

$y: L_{y}=100 \mathrm{~km}=O\left(10^{7} \mathrm{~cm}\right)$

$z: D=$ vertical range of Equatorial Deep Jets, $D=2,000 \mathrm{~m}=O\left(2 \times 10^{5} \mathrm{~cm}\right)$

$\mathrm{u}: \mathrm{U}=\mathrm{O}(20 \mathrm{~cm} / \mathrm{s})$ zonal jets velocity magnitude

$\mathrm{v}: \mathrm{V}=\mathrm{U}=\mathrm{O}(20 \mathrm{~cm} / \mathrm{s})$. Measurements suggest that $\mathrm{v}$ and $\mathrm{u}$ are of same order although $\mathrm{v}$ is more variable and is a little smaller than $\mathrm{u}$.

$w: W=D / L_{y} U$. This relation is from the continuity equation and is due to the fact that $L_{\mathbf{x}}>>L_{y}$ (see below)

$P: P_{0}=\rho_{0} U \beta L_{y}^{2}$ This scale is chosen so that the meridional momentum equation is almost in geostrophic balance. That the zonal velocity could be calculated by geostrophic method was reasonably well confirmed by direct current meter measurement although the noise level was high (Eriksen, 1982). Greg Johnson (private communication) showed me some density and $\mathrm{N}^{2}$ section contours which indicated that there was a trapped density pattern near the equator between $1^{\circ} \mathrm{N}$ and $1^{\circ} \mathrm{S}$, of vertical scale $\mathrm{O}(400 \mathrm{~m})$.

$\bar{\rho}=$ magnitude of density perturbation,

Near the equator, the following approximations are highly accurate $\sin \varphi \sim \varphi=y / a(\beta$-plane approximation)

$\cos \varphi \sim 1$

The nondimensionalized equations are

$$
S \frac{\partial u}{\partial t}+R\left(\delta_{1} u \frac{\partial u}{\partial x}+v \frac{\partial u}{\partial y}+w \frac{\partial u}{\partial z}\right)-y v+\Pi w
$$




$$
\begin{aligned}
&=-\delta_{1} \frac{\partial P}{\partial x}+E_{v} \frac{\partial^{2} u}{\partial z^{2}} \\
& S \frac{\partial v}{\partial t}+R\left(\delta_{1} u \frac{\partial v}{\partial x}+v \frac{\partial v}{\partial y}+w \frac{\partial v}{\partial z}\right)+y u \\
&=-\frac{\partial P}{\partial y}+E_{v} \frac{\partial^{2} v}{\partial z^{2}} \\
& \delta_{2}^{2} S \frac{\partial w}{\partial t}+\delta_{2}^{2} R\left[\delta_{1} u \frac{\partial w}{\partial x}+v \frac{\partial w}{\partial y}+w \frac{\partial w}{\partial z}\right]-\Pi u \\
&=-\frac{\partial P}{\partial z}-F \rho+\delta_{2}^{2} E_{v} \frac{\partial^{2} w}{\partial z^{2}} \\
& \delta_{1} u_{x}+v_{y}+w_{z}=0 \\
& S_{1} \frac{\partial \rho}{\partial t}+\delta_{1} u \frac{\partial \rho}{\partial x}+v \frac{\partial \rho}{\partial y}+w \frac{\partial \rho}{\partial z}+\frac{H}{\rho} \frac{\partial \rho_{0}}{\partial z} w \\
&=K_{v} \frac{\partial^{2} p}{\partial z^{2}}
\end{aligned}
$$

where

$$
\begin{array}{ll}
S=\frac{1}{\beta L_{y} T} & R=\frac{U}{\beta L_{y}^{2}} \\
\delta_{1}=L_{y} / L_{x} & \delta_{2}=D / L_{y} \\
E_{v}=\frac{A_{v}}{D^{2} \beta L_{y}} & \Pi=\frac{f_{0} D}{\beta L_{y}^{2}} \\
F=\frac{\overline{\rho g D}}{\rho_{0} U \beta L_{y}^{2}} & S_{1}=\frac{L_{y}}{T U} \\
F_{v}=\frac{K_{v} L_{y}}{U D^{2}} &
\end{array}
$$

With typical EDJ scale and the following parameter values: $\mathrm{a}=\mathrm{O}\left(6.4 \times 10^{3} \mathrm{~km}\right)=$ $O\left(6.4 \times 10^{8} \mathrm{~cm}\right), f_{0}=2 \Omega=1.4 \times 10^{-4} \mathrm{rad} / \mathrm{sec}$ (the value of horizontal Coriolis parameter at equator), $\beta=2 \times 10^{-13} \mathrm{rad} /(\mathrm{cm} \mathrm{s}), A_{v}=K_{v}=O\left(10^{2} \mathrm{~cm}^{2} / \mathrm{s}\right.$ ) (The estimates of $A_{v}$ and 
$\mathrm{K}_{\mathrm{v}}$ have many uncertainties; we temporarily choose this order in the calculation of nondimensional numbers). Then, we have

$$
\begin{array}{ll}
S=3.3 \times 10^{-3} & R=1.0 \\
\delta_{1}=10^{-2} & \delta_{2}=2 \times 10^{-2} \\
E_{v}=10^{-3} & S 1=8 \times 10^{-3} \\
\Pi=1.0 & F_{v}=10^{-3}
\end{array}
$$

where $R$ and $\Pi$ characterize the size of advective nonlinearity and the horizontal Coriolis parameter related acceleration terms in the equations. It is seen that in the equatorial deep jets regime, neither of these two effects can be neglected at least by scaling argument.

In the limit $S \rightarrow 0, S_{1} \rightarrow 0, \delta_{1} \rightarrow 0, \delta_{2} \rightarrow 0$, we have our simplified equations

$$
\begin{aligned}
& R\left(v \frac{\partial u}{\partial y}+w \frac{\partial u}{\partial z}\right)-y v+w=E_{v} \frac{\partial^{2} u}{\partial z^{2}} \\
& R\left(v \frac{\partial v}{\partial y}+w \frac{\partial v}{\partial z}\right)+y u=-\frac{\partial P}{\partial y}+E_{v} \frac{\partial^{2} v}{\partial z^{2}} \\
& u=\frac{\partial P}{\partial z}+F \rho \\
& \frac{\partial v}{\partial y}+\frac{\partial w}{\partial z}=0 \\
& v \frac{\partial \rho}{\partial y}+w \frac{\partial \rho}{\partial z}+k w=F_{v} \frac{\partial^{2} \rho}{\partial z^{2}} \text { where } k=\frac{H}{\rho} \frac{\partial \rho_{0}}{\partial z}
\end{aligned}
$$

This is the governing equation set for equatorial deep jets. $F$ is hard to estimate because we do not have the magnitude of the density anomaly which is associated with deep jet flow. $\mathrm{F} \rightarrow 0$ implies that the density field can be determined passively from the known velocity field. This decoupling between the density equation and the momentum equation greatly simplifies the mathematical complexity. For a homogeneous ocean, this decoupling is always true. However, in all of our discussions, $\mathrm{F}$ is not zero.

The density equation suggests that the conventionally approximated equation usually uned in wave model $\left(\frac{\partial \rho}{\partial t}+w \frac{\partial \rho_{0}}{\partial z}=0\right)$ overemphasizes the importance of the vertical stratification in temporal density variations in the case of interest. For very low 
frequency motion, effects of advective nonlinearity and diffusion of density need to be considered.

The zonal pressure gradient is ignored after using the scaling argument. From observations we know that there is a strong $P_{x}$ in the upper $200 \mathrm{~m}$ of equatorial ocean, which is believed to be generated by westward trade wind and drives the Equatorial Undercurrent. However, under the thermocline, the gross isothermal lines are almost horizontal. There is no evidence that on a basin wide scale the zonal pressure gradient below the thermocline is significant.

The assumption of no zonal variation is the approximation which was also used in many undercurrent models (Stommel, 1960, Charney, 1960) although the zonal pressure gradient was assumed to be constant in Charney's model. These models also assumed that the surface layer was essentially decoupled from the deep ocean. The thermocline works as a solid wall which blocks the mass and momentum transfer to the deep ocean. To study deep ocean phenomena, this block has to be removed.

Most equatorial dynamical models neglect the horizontal Coriolis parameter effect. One of the reasons for this is probably that these models were developed to model the equatorial undercurrent and other surface trapped currents. The vertical scale associated with them is so small that the horizontal Coriolis parameter is not important. However, for the equatorial deep jets, although the vertical scale for any one jet is small, the overall vertical extension of multiple jets is large, so the effect may not necessarily be neglected.

The next section is a general discussion of the horizontal Coriolis parameter from the point of view of the conservation laws of angular momentum and of kinetic energy. In section 5 and section 6 , an inviscid case of governing equations (3.4.a)-(3.4.e) is discussed. A so called potential zonal vorticity conservation equation is obtained and a nonlinear equatorial deep jet model is developed. 


\section{Section 4. General discussion on the horizontal Coriolis parameter}

The relative importance of two the Coriolis acceleration terms in the zonal momentum equation is

$$
\gamma=\frac{2 \Omega \cos \varphi w}{2 \Omega \sin \varphi v}=\frac{\cos \varphi w}{\sin \varphi v}
$$

where the numerator is associated with the horizontal Coriolis parameter and the denominator is associated with the vertical Coriolis parameter. After using the following approximation close to the equator

$$
\begin{aligned}
& \cos \varphi \sim 1 \\
& \sin \varphi \sim \varphi=y / a
\end{aligned}
$$

where $a$ is the radius of earth, then

$$
\gamma=\frac{a w}{y v}
$$

For many equatorial phenomenon, the zonal scale is very long, and the scale of $w$ can be obtained from the continuity equation as

$$
\mathrm{O}(\mathrm{W})=\mathrm{O}\left(\frac{\mathrm{H}}{\mathrm{L}_{\mathrm{y}}} \mathrm{U}\right)
$$

where $\mathrm{H}$ is defined as the depth of the ocean, $\mathrm{L}_{\mathrm{y}}$ is the meridional scale of the motion.

Now

$$
\gamma=\frac{a}{y} \frac{H}{L_{y}}
$$

when $y$ is small, close to the equator, $\gamma>1$, and when $y$ is large, away from equator, $\gamma<<1$. We define a meridional scale $\mathrm{L}_{y}$ as the distance away from the equator where the two Coriolis terms are of same importance.

$$
\begin{gathered}
\gamma=\frac{\mathrm{aH}}{\mathrm{L}_{\mathrm{y}}^{2}}=1 \\
\mathrm{~L}_{y}=\sqrt{\mathrm{aH}}
\end{gathered}
$$


For typical oceans, $a=6.4 \times 10^{6} \mathrm{~m}, \mathrm{H}=5 \times 10^{3} \mathrm{~m}$, then $\mathrm{L} y=180 \mathrm{~km}$, which is about $1.5^{\circ} \mathrm{N}$ or $1.5^{\circ} \mathrm{S}$ away from equator. For the atmosphere, if we choose height scale, $\mathrm{H}=$ $18 \mathrm{~km}$ then $\mathrm{L}_{\mathrm{y}}=340 \mathrm{~km}$, which is about $3^{\circ} \mathrm{N}$ or $3^{\circ} \mathrm{S}$ away from equator. The above argument seems to suggest that the Coriolis component associated with the horizontal component of earth's rotation $2 \Omega \cos \varphi$ is not negligible within a significant meridional band $\left(-\mathrm{L}_{\mathrm{y}}, \mathrm{L}_{\mathrm{y}}\right)$. To study this in more detail, let us look at its role from the point of the view of the principle of angular momentum conservation.

The exact angular momentum principle is

$$
\frac{d}{d t}[r \cos \varphi(u+\Omega r \cos \varphi)]=F_{\lambda} r \cos \varphi
$$

which can be obtained from the zonal momentum equation

where

$$
\begin{aligned}
& \frac{\mathrm{du}}{\mathrm{dt}}=\mathrm{F}_{\lambda}+\left(2 \Omega+\frac{\mathrm{u}}{\mathrm{r} \cos \varphi}\right)(\mathrm{v} \sin \varphi-\mathrm{w} \cos \varphi) \\
& \frac{\mathrm{d}}{\mathrm{dt}} \equiv \frac{\partial}{\partial \mathrm{t}}+\frac{\mathrm{u}}{\mathrm{r} \cos \varphi} \frac{\partial}{\partial \lambda}+\frac{\mathrm{v}}{\mathrm{r}} \frac{\partial}{\partial \varphi}+\mathrm{w} \frac{\partial}{\partial \mathrm{r}}
\end{aligned}
$$

$F_{\lambda}$ is the total zonal external forcing exerted on fluid element under consideration.

A dimensional conservative form of Eq.(3.4.a) can be written as

$$
\frac{\mathrm{d}^{\prime}}{\mathrm{dt}}\left[\mathrm{u}-\frac{\Omega}{\mathrm{a}} \mathrm{y}^{2}+2 \Omega \mathrm{z}\right]=\mathrm{F}_{\mathrm{x}}
$$

where $\frac{d^{\prime}}{d t} \equiv v \frac{\partial}{\partial y}+w \frac{\partial}{\partial z}$ and the coordinate system is set so that $z$ is positive upwards and $z=0$ at ocean surface, $y=0$ at equator and $y$ is positive northward. It can be seen that if the term $2 \Omega \cos \varphi w$ is neglected in the zonal momentum equation, then the third term on the left hand side of (4.10) $2 \Omega z$ will disappear at the same time. Equation (4.10) means that the angular momentum $u-\frac{\Omega}{a} y^{2}+2 \Omega z$ is constant along a streamline in the meridional plane if $F_{X}=0$.

Under the scale of interest, we can also derive (4.10) directly from (4.8). Write $r=$ $a+z$, where $a$ is the radius of the earth defined as the distance from the center of the earth to ocean surface, for shallow ocean $z<<a$. Noticing that 


$$
\cos \varphi \simeq 1-\frac{\varphi^{2}}{2} \text { for } \varphi=y / a<<1
$$

the angular momentum in the argument of (4.8) can be approximated as

$$
\begin{aligned}
& \operatorname{rcos} \varphi(\mathrm{u}+\Omega \operatorname{rcos} \varphi) \\
& =(\mathrm{a}+\mathrm{z})\left(1-\frac{\varphi^{2}}{2}\right) \mathrm{u}+\Omega(\mathrm{a}+\mathrm{z})^{2}\left(1-\frac{\varphi^{2}}{2}\right)^{2} \\
& =\mathrm{au}+\Omega\left(\mathrm{a}^{2}+2 \mathrm{az}\right)\left(1-\varphi^{2}\right)+O\left(\mathrm{z}^{2}, \mathrm{zu}, \mathrm{z} \varphi^{2}, \varphi^{4}\right) \\
& \approx \Omega \mathrm{a}^{2}+\mathrm{a}\left[\mathrm{u}-\frac{\Omega}{\mathrm{a}} \mathrm{y}^{2}+2 \Omega \mathrm{z}\right]
\end{aligned}
$$

Apart from an additive constant $\Omega \mathrm{a}^{2}$ and a multiplication constant a, the conserved identity is just $\left[u-\frac{\Omega}{a} y^{2}+2 \Omega z\right]$, which is exactly the same argument on the left hand side of (4.10). Therefore, (4.10) is consistent with the principle of angular momentum . conservation if the equatorial $\beta$-plane approximation and the shallow water approximation $\mathrm{H} / \mathrm{a}<<1$ hold. In another words, to have a consistent angular momentum conservation principle, $2 \Omega z$ should be kept in the approximate angular momentum expression. Far from the equator, $2 \Omega z$ is much smaller than $\frac{\Omega}{\mathrm{a}} \mathrm{y}^{2}$ and the latter term dominates. The latitude at which these two terms are of the same order is

$$
\frac{\Omega}{a} L_{y}^{2}=2 \Omega H \rightarrow L_{y}=\sqrt{2 \Omega H}
$$

which is the same to (7) apart from a factor of $\sqrt{ } 2$.

If the projection of a three dimensional particle path on the meridional plane is a line A-B-C as shown in Fig.4.1, then this line is a streamline on that plane. A fluid particle moves upward from $A$ in equatorial deep ocean to near surface $B$, then moves poleward to position C. Denoting the vertical and horizontal excursions as $\Delta z$ and $\Delta y$ respectively, and assuming $F_{x}=0$, there is no net zonal angular momentum torque forcing, the zonal velocity at $A$ and $C$ relative to $u(B)$ are

$$
\begin{aligned}
& u(C)-u(B)=\frac{\beta(\Delta y)^{2}}{2} \\
& u(A)-u(B)=f_{0} \Delta z
\end{aligned}
$$


Equation 4.14.a is just the simplest form of the conservation of vertical potential vorticity (planetary vorticity in this case). It approximately explains the trapping of the equatorial undercurrent according to Fofonoff and Montgomery (1955) and Pedlosky (1987). For $\Delta y$ $=350 \mathrm{~km}, u(C)-u(B)=120 \mathrm{~cm} / \mathrm{s}$, this value is very close to the magnitude of the equatorial undercurrent velocity. For $f_{0}=2 \Omega=1.4 \times 10^{-4} \mathrm{rad} / \mathrm{sec}, \Delta z=5 \mathrm{~km}$, a velocity difference between ocean surface and ocean bottom is about $70 \mathrm{~cm} / \mathrm{s}$. The same amount of velocity difference can be attained by a meridional excursion of $\Delta y=250 \mathrm{~km}$. Of course, the vertical excursion $\Delta z$ of real motion will not be as large as the ocean depth generally. For example, the presence of the stratification will prevent so large an excursion from occurring. However, the effect of this difference can be strengthened or weakened by the existence of zonal pressure gradients and other zonal forcing. Since the effects in different dynamic models are different, separate discussions in specified situations are needed. The next several sections are devoted to that. A general conciusion, however, is that close to equator, within $|\mathrm{y}|<\sqrt{2} \mathrm{Ha}$, the Coriolis acceleration term $2 \Omega \cos \varphi \mathrm{w}$ cannot be neglected in the momentum equation if the angular momentum conservation principle is to be satisfied.

From all three governing momentum equations, we can obtain an energy conservation principle by multiplying the zonal, meridional and vertical momentum equations by $u, v, w$, respectively and then summing

$$
\frac{d}{d t}\left(\frac{u^{2}}{2}+\frac{v^{2}}{2}+\frac{w^{2}}{2}\right)=-\vec{u} \cdot \nabla P+\vec{u} \cdot \vec{F}
$$

It can be seen that if the Coriolis acceleration term $-2 \Omega \cos \varphi \mathrm{u}$ in the vertical momentum equation is neglected, then the energy equation (4.15) will be incomplete. There would be an extra term $-2 \Omega \cos \varphi$ uw on the right hand side of (4.15). Although the inaccuracy maybe small, after a long time integration of the governing equation the distortion of fluid motion due to the small incompleteness in energy conservation may be significant. In the parameter range of interest, ( see Section 3), this incompleteness is significant. So we reach another conclusion, to satisfy the energy conservation equation, the vertical Coriolis acceleration 
term should be retained in the vertical momentum equation as long as $2 \Omega \cos \varphi w$ is important in the zonal momentum equation.

Most work on equatorial dynamics has concentrated on the upper ocean (shallower than $300 \mathrm{~m}$ ) phenomena, so the effect of horizontal Coriolis parameter is very weak because of the short vertical ranges. However, many papers scaled the horizontal Coriolis parameter to be negligible, even when the vertical scale was chosen to be the ocean depth, as in Moore and Philander (1976). The reason may be due to their special choice of meridional length scale $L_{x}=L_{y}$. It would not be very proper to scale many strongly meridionally trapped narrow equatorial currents including deep jets as $\mathrm{L}_{\mathbf{x}}=\mathrm{L}_{\mathbf{y}}$.

The equatorial region where the horizontal Coriolis parameter is significant is small, about $1^{\circ} \mathrm{N} \sim 1^{\circ} \mathrm{S}$, and the analysis of the fluid dynamics within this region may be very different from that outside. In mid-latitude $f=2 \Omega \sin \phi_{0}+\beta y$, the two parameters $2 \Omega \sin \phi_{0}$ and $\beta$ are all important, and $2 \Omega \sin \phi_{0}$ is the principal factor which satisfies the geostrophic relation approximately. While at the equator, the $\beta$ effect is dominant, $2 \Omega \sin \phi_{0}$ $\rightarrow 0$.The closer to the equator, the larger is $\beta$. The $\beta$-effect is more significant in low latitude than in high latitude. This may be the reason why that in low latitudes, the flow tends to be more zonal than in mid-latitudes. The main feature of the equatorial dynamics is that as the vertical Coriolis parameter approaches zero, the horizontal Coriolis parameter reaches a maximum of $2 \Omega$ as the equator is approached. 


\section{Section 5. Potential zonal vorticity conservation}

To have the physical meaning clearer during the mathematical derivation, we will use the dimensional variable formulations. From (3.1.a) - (3.1.e), by assuming two important equatorial deep jets features, $\frac{\partial}{\partial t} \equiv 0, \frac{\partial}{\partial x} \equiv 0$, and neglecting dissipation, we have

$$
\begin{aligned}
& v \frac{\partial u}{\partial y}+w \frac{\partial u}{\partial z}-\beta y v+f_{0} w=0 \\
& v \frac{\partial v}{\partial y}+w \frac{\partial v}{\partial z}+\beta y u=-\frac{1}{\rho_{0}} \frac{\partial P}{\partial y} \\
& v \frac{\partial w}{\partial y}+w \frac{\partial w}{\partial z}-f_{0} u=-\frac{1}{\rho_{0}} \frac{\partial P}{\partial z}-\frac{\rho}{\rho_{0}} g \\
& v \frac{\partial \rho}{\partial y}+w \frac{\partial \rho}{\partial z}+w \frac{\partial \rho_{0}}{\partial z}=0 \\
& \frac{\partial v}{\partial y}+\frac{\partial w}{\partial z}=0
\end{aligned}
$$

where $\frac{\partial \rho_{0}}{\partial z}$ is the mean background vertical density gradient, a function of $z$ only.

It is easy to rewrite (5.1.a) and (5.1.d) as

$$
\begin{aligned}
& \left(v \frac{\partial}{\partial y}+w \frac{\partial}{\partial z}\right)\left(u-\frac{\beta y^{2}}{2}+f_{0} z\right)=0 \\
& \left(v \frac{\partial}{\partial y}+w \frac{\partial}{\partial z}\right)\left(\rho+\int \frac{\partial \rho_{0}}{\partial z} d z\right)=0
\end{aligned}
$$

Eq. (5.2) and (5.3) are angular momentum conservation and total density conservation respectively. The zonal angular momentum consists of three parts, the pure zonal velocity relative to the earth, and the effects associated with the vertical component and the horizontal component of the earth's rotation. The sum of the latter two parts may be 
called the planetary zonal velocity, $u_{p}=-\frac{\beta y^{2}}{2}+f_{0} z$ since both of them are related to the earth's rotation.

If we define a streamfunction $\varphi$ in the meridional plane as

$$
v=-\frac{\partial \varphi}{\partial z}, w=\frac{\partial \varphi}{\partial y}
$$

then,

$$
\begin{aligned}
& u-\frac{\beta y^{2}}{2}+f_{0} z=Q_{1}(\varphi) \\
& \rho+\int \frac{\partial \rho_{0}}{\partial z} d z=Q_{2}(\varphi)
\end{aligned}
$$

where $Q_{1}(\varphi)$ and $Q_{2}(\varphi)$ are two arbitrary functions of $\varphi$. So in this case, the zonal angular momentum and total density are conserved along streamlines in the $y-z$ plane.

Another conservation equation can be obtained by eliminating the pressure $\mathrm{P}$ from (5.1.b) and (5.1.c) and forming an equation for the zonal component of relative vorticity

$$
\left[v \frac{\partial}{\partial y}+w \frac{\partial}{\partial z}\right]\left(\frac{\partial v}{\partial z}-\frac{\partial w}{\partial y}\right)+\beta y \frac{\partial u}{\partial z}+f_{0} \frac{\partial u}{\partial y}=\frac{g}{\rho_{0}} \frac{\partial \rho}{\partial y}
$$

The zonal component of relative vorticity is

$$
\Omega_{x}=\left(\frac{\partial v}{\partial z}-\frac{\partial w}{\partial y}\right)=-\left(\frac{\partial^{2}}{\partial y^{2}}+\frac{\partial^{2}}{\partial z^{2}}\right) \varphi
$$

Its variation when a fluid particle moves along a streamline in the $y-z$ plane is affected by:

(a) $\beta y \frac{\partial u}{\partial z}+f_{0} \frac{\partial u}{\partial y}$; the scalar product of the Coriolis parameter $f=\left(0, f_{0}, \beta y\right)$ and the zonal velocity shear $\nabla u=\left(0, \frac{\partial u}{\partial y}, \frac{\partial u}{\partial z}\right)$.

(b) $\frac{g}{\rho_{0}} \frac{\partial \rho}{\partial y}$; the meridional gradient of gravity force variation due to the presence of the meridional gradient of density anomaly. 
Notice, by using (5.5)

$$
\begin{aligned}
& \beta y \frac{\partial u}{\partial z}+f_{0} \frac{\partial u}{\partial y} \\
& =\left(\beta y \frac{\partial Q_{1}}{\partial z}+f_{0} \frac{\partial Q_{1}}{\partial y}\right) \\
& =\frac{d Q_{1}}{d \varphi}\left(\beta y \frac{\partial \varphi}{\partial z}+f_{0} \frac{\partial \varphi}{\partial y}\right) \\
& =\frac{d Q_{1}}{d \varphi} J\left(\varphi,\left(-\frac{\beta y^{2}}{2}+f_{0} z\right)\right) \\
& =J\left(\varphi, \frac{d Q_{1}}{d \varphi}\left(-\frac{\beta y^{2}}{2}+f_{0} z\right)\right)
\end{aligned}
$$

where the operator $J(\varphi,$.$) is the Jacobian, and using (5.6)$

$$
\frac{\partial \rho}{\partial y}=\frac{\partial Q_{2}}{\partial y}=\frac{d Q_{2}}{d \varphi} \frac{\partial \varphi}{\partial y}=\frac{d Q_{2}}{d \varphi} J(\varphi, z)=J\left(\varphi, z \frac{d Q_{2}}{d \varphi}\right)
$$

So, equation (5.7) can be transformed into

$$
J\left(\varphi,-\nabla^{2} \varphi+\frac{d Q_{1}}{d \varphi}\left(\frac{\beta y^{2}}{2}+f_{0} z\right)-\frac{g}{\rho_{0}} \frac{d Q_{2}}{d \varphi} z\right)=0
$$

The second argument in (5.11) is a conserved quantity along streamlines in the meridional plane, that is

$$
-\nabla^{2} \varphi+\frac{d Q_{1}}{d \varphi}\left(-\frac{\beta y^{2}}{2}+f_{0} z\right)-\frac{g}{\rho_{0}} \frac{d Q_{2}}{d \varphi} z=Q_{3}(\varphi)
$$

This conserved quantity may be called the zonal potential vorticity and is composed of three parts

(a) $-\nabla^{2} \varphi$, pure kinematic relative zonal vorticity;

(b) $\frac{d Q_{1}}{d \varphi}\left(-\frac{\beta y^{2}}{2}+f_{0} z\right)$, the variation of zonal momentum across meridional streamlines multiplied by planetary zonal velocity;

(c) $-\frac{\mathrm{g}}{\rho_{0}} \frac{\mathrm{d} \mathrm{Q}_{2}}{\mathrm{~d} \varphi} \mathrm{z}$, the variation of total density across meridional streamlines multiplied by the geopotential. 
Equation (5.12) in the absence of Coriolis parameters (the second term on the left hand side of the equation) has been derived by Long (1958) and Yih (1960) for a nonrotating stratified fluid system. The solutions of their linearized equations were discussed in detail and could successfully explain the phenomenon of flow over a barrier and flow into a sink. We will explore the equatorial deep jet solution of the potential zonal vorticity conservation equation (5.12) when the second term is included in the next section. 


\section{Section 6. An inertial equatorial deep jets model}

As reviewed in Section 1, we know that linear equatorial wave models are incapable of fully explaining the deep equatorial deep jets due to the difficulty in showing how wind generated energy propagates down to the deep ocean. The following question was raised. Can this phenomenon be explained as free, inertial motion in the deep ocean below the thermocline? From the scale analysis using observed magnitudes of the phenomenon, it is seen that the advective nonlinearity cannot be simply neglected. Although many equatorial phenomenon can be successfully interpreted by linear wave models, even these phenomena may be in nonlinear range. It is still an interesting question as to what is the effect of nonlinearity. This section proposes a nonlinear inertial model for the equatorial deep jets.

From the last section, we know that steady, zonally independent equatorial ocean dynamics are governed by three first integrations. They are: zonal angular momentum conservation (5.5), total density conservation (5.6) and the potential zonal vorticity conservation (5.12). We expect that the equatorial deep jets can be described by these conservation equations. To discover exact solutions of them, it is natural to consider, as a first step, the circumstances in which the potential zonal vorticity conservation becomes linear in $\varphi$. At this stage, one may have two different approaches. Since the functions $\mathrm{Q}_{1}(\varphi), \mathrm{Q}_{2}(\varphi)$ and $\mathrm{Q}_{3}(\varphi)$ are related to the conditions away from the deep jets, one may either try different far field conditions and see whether these conditions will make the equations linear, or assume the equations to be linear to start with and inquire as to what the corresponding conditions must be. It is difficult for us to follow the first approach since it is as yet unclear what kind of relationship exists between the equatorial deep jets and the extra-equatorial deep flow. It is also not clear how surface wind stress forces the deep flow by penetrating through the equatorial undercurrent. Therefore, we will adopt the second 
approach. This is evidently more exhaustive and more economical if the linearized equations are adequate. Of course, this is an ad hoc assumption. It is equivalent to choosing a type of solution from an infinite number of possible solutions. The most general linear form of Eq.(5.12) is

$$
-\nabla^{2} \varphi+(A \varphi+B)\left(-\frac{\beta y^{2}}{2}+f_{0} z\right)-\frac{g}{\rho_{0}} z(C \varphi+D)=E \phi+F
$$

where three arbitrary functions are chosen as $\frac{d Q_{1}}{d \varphi}=A \varphi+B, \frac{d Q_{2}}{d \varphi}=C \varphi+D, Q_{3}=E \varphi+F$.It is observed that the equatorial deep jets are strongly trapped near equator, and we assume that away from the equator, the meridional and vertical motion will approach zero, $\varphi \rightarrow 0$,

which requires from $(6.1)$

$$
B=D=F=0
$$

At this stage, assumption (6.2) is the best assumption we can make. ( A even stronger condition, is $v \equiv 0$, in the Kelvin wave model.)

Then the corresponding arbitrary functions are

$$
\begin{aligned}
& Q_{1}(\varphi)=\frac{A}{2} \varphi^{2}+Q_{10} \\
& Q_{2}(\varphi)=\frac{C}{2} \varphi^{2}+Q_{20} \\
& Q_{3}(\varphi)=E \varphi
\end{aligned}
$$

From (5.4), (5.5) and (5.6), the zonal velocity, meridional velocity, vertical velocity and density spatial structure can be written as

$$
\begin{aligned}
& u=\frac{\beta y^{2}}{2}-f_{0} z+A \varphi^{2} / 2+u_{0} \\
& v=-\frac{\partial \phi}{\partial z}, w=\frac{\partial \phi}{\partial y} \\
& \rho+\int \frac{\partial \rho_{0}}{\partial z} d z=C \varphi^{2} / 2+\rho_{0}
\end{aligned}
$$

where the constants $u_{0}$ and $\rho_{0}$ are used to replace $Q_{10}$ and $Q_{20}$. They can be obtained after the streamfunction has been computed from the potential zonal vorticity equation. It is 
noticed here that zonal velocity u consists of two major parts, the planetary zonal velocity and a perturbation part. We explore separable solution of (6.1) now under condition (6.2), let

$$
\varphi=\mathrm{Y}(\mathrm{y}) \mathrm{Z}(\mathrm{z})
$$

then the equations for the meridional structure and vertical structure respectively are

$$
\begin{aligned}
& Y^{\prime \prime}+\left(\lambda^{2}+A \frac{\beta y^{2}}{2}\right) Y=0 \\
& Z^{\prime \prime}+\left[E-\lambda^{2}+\left(\frac{g}{\rho_{0}} C-A f_{0}\right) z\right] Z=0
\end{aligned}
$$

The double prime " stands for the second derivative with respect to $y$ and $z$ in (6.8) and (6.9), respectively. The Y structure equation is an eigenvalue problem with Hermite functions as eigensolutions which are all trapped near the equator if condition $Y \rightarrow 0$ as $y$ approaching to infinity is used. Let

$$
\mathrm{y}=\mathrm{L}_{\mathrm{y}} \xi
$$

where $L y$ is a meridional trapping scale, $\xi$ is nondimensional, then

$$
\frac{d^{2} Y}{d \xi^{2}}+\left[\lambda^{2} L_{y}^{2}+A \beta L_{y}^{4 \xi^{2}} / 2\right] Y=0
$$

Having eigensolutions requires

$$
\begin{aligned}
& A \beta L_{y}^{4} / 2=-1 \\
& \lambda^{2} L_{y}^{2}=2 j+1 \quad j=0,1,2,3 \ldots
\end{aligned}
$$

then

$$
Y=Y_{j}=\frac{e^{-\xi^{2} / 2} H_{j}(\xi)}{\left(2^{j} j ! \pi^{1 / 2}\right)^{1 / 2}}
$$

where

$$
H_{j}(\xi)=(-1)^{j} e^{\xi^{2}} \frac{d^{j}}{d \xi^{j}} e^{-\xi^{2}}
$$

Eliminate $L y^{2}$ from (6.12) and (6.13), let $\lambda_{j}$ be the eigenvalue corresponding to the jth mode $Y_{j}$, then

$$
\lambda_{j}^{4}=-\frac{A \beta}{2}(2 j+1)^{2}
$$


We notice that there are many arbitrary constants in this model. These constants can only be determined by fitting the solution to the observed features. So this model is only a qualitative model used to see whether a pure free, inertial equatorial deep jets model is possible. Eq. (6.16) states that the coefficient $A$ has to be negative to have real eigenvalues. The first three horizontal structure eigenfunctions of (6.14) are shown in Fig.6.1.(a), Fig. 6.1.(b) and Fig. 6.1.(c), where $\xi$ is a meridional nondimensionalized coordinate. The trapping scale $L_{y}$ from (6.12) is related with $\beta$ and $A$, where $\beta$ is associated with the earth's sphericity and A characterizes the amplitude of the alternating part of the zonal velocity in (6.4). Because this model is nonlinear, the amplitudes of the velocities can be finite. The vertical structure equation becomes

$$
Z^{\prime \prime}+\left[E-(2 j+1) \sqrt{-\frac{A \beta}{2}}+\left(\frac{g}{\rho_{0}} C-A f_{0}\right) z\right] Z=0
$$

Let

$$
\begin{aligned}
& \delta_{1}=E-(2 j+1) \sqrt{-\frac{A \beta}{2}} \\
& \delta_{2}=\frac{g}{\rho_{0}} C-A f_{0}
\end{aligned}
$$

and change the coordinates such that

$$
\left(\delta_{1}+\delta_{2} z\right)=\alpha^{2} \eta
$$

where the transformed coordinate $\eta$ is positive everywhere to have oscillating solution $Z(z)$. Since $z<0$ everywhere, we need $\delta_{2}<0$. Since $A_{0}<0, C$ must be negative. The effects of the horizontal Coriolis parameter come from two places. A direct addition of constant vertical shear $f_{0 z}$ to the zonal velocity (6.4) and some effect associated with Af $_{0}$ in the vertical structure equation (6.17). Notice from (6.4) and (6.6) that $C$ is the amplitude of density anomaly and that $\mathrm{A}$ is the amplitude of zonal velocity anomaly. The significance of this second effect depends on the magnitude of $A$ and $C$. If the streamfunction is $O(\phi), C$ is of the order of the density anomaly, $O\left(10^{-3} / \phi^{2}\right) \times \rho_{0}$ then $A$ is $O\left(10 \mathrm{~cm} / \mathrm{s} / \phi^{2}\right)$. Thus, 
$\mathrm{gC} / \mathrm{p}_{0}$ is much much larger than $\mathrm{Af}_{0}$. So the effect of horizontal Coriolis parameter in the vertical structure function is not important. The only effect is in the background zonal flow. $\alpha$ can be chosen such that $\eta$ is a nondimensional variable. If $\delta_{1}>0$, the ocean surface $z=0$ corresponds to $\eta=\delta_{1} / \alpha^{2}>0$. Except for a translational change to vertical structure, we can assume $E=0$ without affecting the solution. $E=0$ and $F=0$ in equation (6.1) means that we have assumed constant potential zonal vorticity. This indicates that the potential zonal vorticity does not change in meridional plane for all streamlines. Let

$$
\gamma^{2}=\frac{\alpha^{6}}{\delta_{2}^{2}}
$$

then

$$
\frac{d^{2} Z_{j}}{d \eta^{2}}+\gamma_{j}^{2} \eta Z_{j}=0
$$

here the index $\mathrm{j}$ is attached to the constant $\gamma$ and the vertical structure function $\mathrm{Z}$ since they are $\mathrm{j}$ dependent. Its solution can be obtained as

$$
Z_{j}(\eta)=\eta^{1 / 2}\left[c_{j} J_{1 / 3}\left(\frac{2}{3} \gamma_{j} \eta^{3 / 2}\right)+d_{j} J_{-1 / 3}\left(\frac{2}{3} \gamma_{j} \eta^{3 / 2}\right)\right]
$$

If we impose $\mathrm{Z}=0$ at two fixed depths, possibly representing the bottom of thermocline and the ocean bottom, then (6.17) is also an eigenvalue problem. Here we have too many undetermined constants, so we only examine the qualitative behavior of the structures of the solution. The vertical structure $\mathrm{J}_{1 / 3}$ is shown in Fig.6.2, $\eta$ is a vertical nondimensionalized coordinate, when $\gamma_{j}=1$. This structure oscillates and its amplitude decreases with depth. The oscillating vertical scale is also decreasing with depth. Another root $\mathrm{J}_{-1 / 3}$ is of very similar structure except with a phase difference. The contour of the streamfunctions for the first three horizontal structure functions and the vertical structure function $\mathrm{J}_{1 / 3}$ are shown in Fig.6.3.(a), Fig.6.3.(b) and Fig.6.3.(c). The general solution of the streamfunction is

$$
\varphi=\sum_{j=0}^{\infty} Y_{j}(y) Z_{j}(z)
$$


Note that the solution we have obtained is in a nonlinear flow regime and the amplitudes of all variables need not necessarily be infinitely small. Streamlines, $\varphi$ are the contour lines in the meridional plane corresponding to the projection of fluid particles in three dimensional space onto the meridional plane. It is clearly equatorially trapped. The vertical structure is oscillating with depth. The vertical scale decreases with depth and the amplitude also decreases with depth.

The meridional velocity and vertical velocity contours in the meridional plane for the first mode are shown in Fig. 6.4 and Fig. 6.5. Notice that the meridional velocity becomes larger in the deep ocean as the vertical scale of the streamlines becomes smaller. This may not be realistic. However, our model neglected the effects of diffusion. Diffusion has a more significant effect on deeper small scale motions. The model shows that the vertical scale gets smaller in the deeper ocean, so the diffusion can dissipate the deep features away. Thus the inclusion of dissipation may prevent the meridional velocity from increasing with depth. The density anomaly structure is shown in Fig.6.6. It is oscillating with respect to a mean which is increasing with respect to depth. This structure is not necessarily unstable due to the presence of an acceleration toward the center of curvature in the cell trajectory in the meridional plane. This density anomaly is also trapped at the equator and with a vertical oscillating scale the same as that of the zonal velocity.

We can still see some features of EDJ in the very deep ocean from the observations, but they are quite obscure. The effect of the bottom on the EDJ is not clear. It seems that different dynamics are involved near the ocean bottom. So, a bottom boundary condition is hard to propose. A similar problem occurs in the upper ocean for the upper boundary condition. We know that the Equatorial Undercurrent above the EDJ and the other currents like the North Equatorial Current, South Equatorial Current, North Equatorial Counter Current and South Equatorial Counter Current all complicate the problem. It is believed that surface wind stress and the associated surface zonal pressure gradient are important for these surface or near surface current systems, especially the 
Equatorial Undercurrent. The connection between this system and the equatorial deep jets is not clear. So a suitable upper boundary condition is not available. Even the meridional condition we have used as $\varphi \rightarrow 0$ is not completely unarguable. These difficulties prevented us from choosing the arbitrary functions $Q_{1}, Q_{2}$ and $Q_{3}$ more realistically and from determining the constants in the expressions for velocity and density more rigorously. We can only describe the spatial structures of these variables in a very qualitative way.

The full solution of $\varphi$ comprises many modes. All of these modes are equatorially trapped and alternating in the vertical. The projection of motion in the meridional plane appears as multiple cells. Higher modes correspond to more cells in the y direction. It is seen from the horizontal structure equation (6.11) that the $\beta$-effect is the main reason for trapping.

The zonal velocity, $u$, of (6.4) is composed of four additive parts.

(1) $\beta y^{2} / 2$, the contribution of the vertical Coriolis parameter in the zonal momentum equation. It increases with latitude, the distance away from equator. So this zonal velocity part is eastward away from equator relative to the flow on the equator. If $L_{y}=100 \mathrm{~km}=10^{7}$ $\mathrm{cm}$. This part is $\mathrm{O}(10 \mathrm{~cm} / \mathrm{s})$.

(2) $f_{0} z$, the contribution of the horizontal parameter in the zonal velocity. This provides a constant vertical shear. The deeper fluid moves eastward relative to the particle above it. If $z=1 \mathrm{~km}=10^{5} \mathrm{~cm}$, this part is $O(10 \mathrm{~cm} / \mathrm{s})$, which is significant.

(3) $u_{0}$, a constant which can not be determined here.

(4) $\mathrm{A} \varphi^{2} / 2$. The zonal velocity part associated with the meridional motion. It is oscillating vertically about a mean which is increasingly westward in shallower ocean. If $f_{0} \equiv 0$, this large scale mean shear will disappear, but the alternating anomaly part still exist.

$P^{1}$ anetary velocity is defined as $u_{p}=\frac{\beta y^{2}}{2}-2 \Omega z$ in section 5 since both terms are associated with the rotation of the earth. The angular momentum conservation states that for 
stationary, zonally independent equatorial flow, the zonal velocity has a background contribution up. Since the dynamics of the upper equatorial ocean is controlled by surface forcing, the westward trade wind acts as a main driving force to the equatorial upper ocean current system. The background zonal velocity up makes sense only in the deep equatorial ocean below the thermocline and very close to the equator ( a requirement of the $\beta$-plane approximation). The free inertial model shows that the equatorial deep jets are superimposed on this background mean planetary flow. The following is to review some observations which are consistent with this result.

The planetary zonal velocity is composed of two parts. The first part is a parabolically distributed zonal velocity in the meridional plane. The zonal velocity of the fluid away from the equator should move eastward relative to the fluid motion on the equator. The second term is associated with a vertical shear. The zonal velocity (positive refers to eastward) increases for the deeper flow. The deeper fluid moves eastward relative to the zonal motion of shallower fluid.

(a) Indian Ocean.

Fig.6.7 shows the zonal velocity profiles observed by Luyten \& Swallow (1976) in equatorial Indian ocean. These profiles are made from stations distributed on the equator. It is seen that a mean vertical shear exists for all profiles in the upper $2 \mathrm{~km}$.

Fig.6.8 is from Ponte and Luyten (1990), (a) and (b) are observed three month apart. It is very clear that a mean vertical shear exists for all profiles in the upper $3000 \mathrm{~m}$. (b) Pacific Ocean.

Fig.1.1 is from Firing (1987). This is an average of zonal velocity over 21 cruises. The shaded area moves westward. The unshaded area moves eastward. It is seen that there is a mean vertical shear. The fluids at depths between $200 \mathrm{~m}$ and $1000 \mathrm{~m}$ very close to the equator generally move westward, while the fluids below $1500 \mathrm{~m}$ generally move eastward. It can also be seen that at latitudes, $1.5^{\circ} \mathrm{N}$ and $1.5^{\circ} \mathrm{S}$, there are two big blocks of fluids moving eastward. This can fairly well be described as planetary zonal velocity. 
Fig.1.2 and Fig.1.3 are taken from Ponte and Luyten (1989). They are zonal velocity profiles along equator (Fig.1.2) and across equator (Fig.1.3). Fig.1.2 again shows a mean vertical shear in the upper $3000 \mathrm{~m}$ of the oceans. The shears in all profiles are highly correlated. Fig.1.3 shows the profiles of stations along a latitudinal section at $138^{\circ}$ W. The five profiles close to equator show the vertical shear in the upper $2000 \mathrm{~m}$. The other two profiles located on $2^{\circ} \mathrm{N}$ and $2^{\circ} \mathrm{S}$ both show a eastward flow in the upper $1000 \mathrm{~m}$.

Fig. 6.9 (Hisard et al, 1970) and Fig.6.10 (Wyrtki \& Kilonsky, 1984) are contours of zonal velocity along meridional sections. The measurements are too shallow, only extending to $500 \mathrm{~m}$ and $400 \mathrm{~m}$, respectively. However, the eastward flows can still be seen about $1.5^{\circ}$ away from equator. The upper portions of these two eastward flows are sometimes called subsurface counter currents and are usually below thermocline extending to depth.

(c) Atlantic Ocean.

Fig.6.11 (Ponte, et al. 1990) was the only profile available to us for the Atlantic Ocean which also indicates a mean vertical zonal velocity shear on the equator.

Equatorial planetary zonal velocity is widely observed in all three major equatorial oceans. This is the background large scale (relative to the size of the deep jets vertical scale) mean motion. Thus observation confirms one of the results of the inertial model (section 6), that the equatorial deep jets zonal velocity signal is superimposed on a mean background zonal planetary velocity. Although most measurements mentioned above are of short temporal duration, the equatorial planetary zonal velocity patterns can be seen in all of these observations.

So, the zonal flow is oscillating vertically relative to a larger scale mean motion caused by the planetary zonal velocity and a part from the anomaly itself. The small vertical scale part is also trapped near the equator.

Although it is impossible to determine all the arbitrary constants in the model due to insufficient information on boundary conditions and far field conditions, we can do a 
consistency check since most of these constants are interrelated. The strategy is to suppose that a single mode can inteprete the equatorial deep jets, and assume the magnitude of the merional streamfunction is of order $\phi=\mathrm{O}\left(5 \times 10^{6} \mathrm{~cm}^{2} / \mathrm{s}\right)$, and then calculate the magnitude of the other variables based on some known information to see if these magnitudes are close to reality.

From (6.5), the magnitudes of the meridional and vertical velocities can be evaluated as $\mathrm{O}(\mathrm{v})=\mathrm{O}(\phi / \mathrm{H})=\mathrm{O}\left(\left(5 \times 10^{6}\right) /\left(5 \times 10^{5}\right)\right)=\mathrm{O}(10 \mathrm{~cm} / \mathrm{s}), \mathrm{O}(\mathrm{w})=\mathrm{O}(\phi / \mathrm{L} \mathrm{y})=$ $\mathrm{O}\left(5 \times 10^{6} / 10^{7}\right)=\mathrm{O}(0.5 \mathrm{~cm} / \mathrm{s})$. The vertical alternating scale can be estimated in the following manner. From the vertical structure Fig.6.2, it can be seen that the alternating scale in the nondimensional variable $\eta$ is $O(1)$. Then from (6.20), the dimensional vertical scale is $\mathrm{L}_{\mathrm{z}}=\alpha^{2} / \delta_{2}$. Since $\gamma=1$ is used in the plot, $\alpha^{2}=\delta_{2}^{2 / 3}$ from the relation above (6.21). $\delta_{2}=\mathrm{gC} / \rho_{0}$ from (6.19) because the second part is very small as justified before. Then, $\mathrm{L}_{\mathrm{z}}=1 / \delta_{2}{ }^{1 / 3}=\left(\mathrm{gC} / \mathrm{\rho}_{\mathrm{o}}\right)-1 / 3=\mathrm{O}\left(\mathrm{gx} 10^{-3} / \phi 2\right)^{-1 / 3}$. If we plug in $\mathrm{g}=10^{3} \mathrm{~cm} / \mathrm{s}^{2}$ and $\phi=O\left(5 \times 10^{6} \mathrm{~cm}^{2} / \mathrm{s}\right)$, then $\mathrm{L}_{2}=\mathrm{O}\left(3 \times 10^{4} \mathrm{~cm}\right)$. Suppose the perturbed part of the zonal velocity (6.4) is of order $\mathrm{O}(10 \mathrm{~cm} / \mathrm{s}), A \phi^{2}=O(10 \mathrm{~cm} / \mathrm{s})$, then $A=0.4 \times 10^{-12} \mathrm{~s} / \mathrm{cm}^{3}$. Then, the meridional e-folding scale $\mathrm{L}_{y}$ can be estimated from $(6.12)$ as $\mathrm{L}_{\mathrm{y}}=\mathrm{O}\left((2 / \beta \mathrm{AA})^{1 / 4}\right)=2 \times 10^{6} \mathrm{~cm}$.

Therefore, it is seen that if we assume the equatorial deep jets are a single mode of the free, inertial motions, the magnitude of all the variables except the meridional length scale can be reasonably well fitted with observations. The model gives a meridional length scale about five times smaller than that observed in reality. Several factors could modify the meridional scale, including changes in the arbitrary chosen functions, and the presence of dissipation. Exploration of these effects is beyond the scope of the present work.

This model in the nonlinear flow range presents some observed equatorial deep jets features. However, it still has several defects. The velocity and density structures are still arguable compared to observations. Both the horizontal and vertical viscous effects, the thermodynamic forcing effect and the zonal variation effect are all neglected in this model. However, it does show that the interpretation of equatorial deep jets in terms of free, 
inertial flow is possible, if more physical factors are included and more observational data are known to provide better choice of the arbitrary functions. It is the advective nonlinearity which is responsible for the existence of the alternating deep jets. To further study this phenomenon, analytical solutions of the zonal potential vorticity equation with the different types of conservative functions are needed. A complete understanding would require numerical computation. For a three dimensional steady numerical model, very few zonal grid points or modes are needed. 


\section{Section 7. Role of horizontal Coriolis parameter in linear equatorial waves}

It is argued in Section 2. that the role of the horizontal Coriolis parameter in equatorial dynamics is not very clear. We will examine its role in two linear cases. (1) inviscid three dimensional low frequency waves. and (2) steady, viscous linear motion. Using the traditional approximation, equatorially trapped waves have been studied for many years (Matsuno 1966, Moore and Philander, 1977). They have also been observed in equatorial oceans (Wunsch and Gill, 1976). The traditional approximation is difficult to justify a priori, although some observations and some a posteriori justifications suggest that the horizontal Coriolis parameter is not important. But when the equator is approached, the neglected Coriolis acceleration terms are not necessarily smaller than the vertical Coriolis parameter-related terms. It is expected that very close to equator, the dynamics would be somewhat different from the dynamics when the traditional approximation is made. It is interesting to look at the character and size of the differences.

For infinitesimal small amplitude, inviscid waves, we have governing equations uniformly valid as follows:

$$
\begin{aligned}
& u_{t}-\beta y v+f_{0} w=-P_{x} / \rho_{0} \\
& v_{t}-\beta y u=-P_{y} / \rho_{0} \\
& -f_{0} u=-\frac{1}{\rho_{0}} P_{z}-\frac{\rho}{\rho_{0}} g \\
& u_{x}+v_{y}+w_{z}=0 \\
& \rho_{t}+w \rho_{0 z}=0
\end{aligned}
$$

The notation $f_{0}=2 \Omega$ is again used. Very close to the equator

$$
\begin{aligned}
& 2 \Omega \cos \varphi \approx 2 \Omega \\
& 2 \Omega \sin \varphi \approx 2 \Omega \varphi=2 \Omega \mathrm{y} / \mathrm{a}=\beta \mathrm{y} .
\end{aligned}
$$


These approximations are very good for $y / a<<1$ to within $5^{\circ}$ north or south away from the equator. We notice that the vertical momentum equation is not a hydrostatic relation and the time derivative of $w$ is neglected. For low frequency waves, periods are longer than $1 / 2 \Omega$, and $w \ll u$, so $w_{t} \ll<f_{0} u$. The Boussinesq approximation has been used. To make the physical meaning of all terms clear during analysis, we still use dimensional variables. The zonal vorticity equation can be constructed by eliminating variable $P$ from (7.1) and (7.2), we have

$$
\left(v_{x}-u_{y}\right)_{t}+\beta y u_{x}+\beta y v_{y}+\beta v-f_{0} w_{y}=0
$$

Also eliminating $P$ from (7.1) and (7.3), results in

$$
u_{z t}-\beta y_{z}+f_{0}\left(u_{x}+w_{z}\right)=\frac{\rho_{x}}{\rho_{0}} g
$$

By using (7.5) and (7.7), the density $\rho$ can be eliminated

$$
u_{z t t}-\beta y v_{z t}+f_{0}\left(u_{x}+w_{z}\right)_{t}=N^{2} w_{x}
$$

where $N=\sqrt{\frac{g}{\rho_{0}} \rho_{0 z}}$ is the Brunt Väisälä frequency. In the following study, it is assumed that $\mathrm{N}^{2}$ is constant. This assumption is not generally valid for the real ocean, but it should be sufficient to fulfill the purpose of only making the comparisons here.

We assume our solution is of form

$$
\left(\begin{array}{l}
u \\
v \\
w
\end{array}\right)=\left(\begin{array}{l}
\bar{u} \\
\bar{v} \\
\bar{w}
\end{array}\right) e^{i(\sigma t+k x+m z)}
$$

Then (7.4), (7.6), (7.8) give

$$
\begin{aligned}
& (i k \bar{v}-\bar{u} y) i \sigma+\beta y \bar{u} i k+\beta y \bar{v}_{y}+\beta \bar{v}-f_{0} \bar{w}_{y}=0 \\
& i k \bar{u}-\bar{v}_{y}+i m \bar{w}=0 \\
& -i m \sigma^{2} \bar{u}+\beta y m \sigma \bar{v}-f_{0} i \sigma v_{y}=i k N^{2} \bar{w}
\end{aligned}
$$

Eliminating $\bar{w}$ from these three equations, we have

$$
\begin{aligned}
& -\sigma m\left(i k \bar{v}-\bar{u}_{y}\right)-k m \beta y \bar{u}+i m \beta y \bar{v}_{y}+i m \beta \bar{v}+f_{0}\left(i k \bar{u}+\bar{v}_{y}\right)_{y}=0 \\
& -i m^{2} \sigma^{2} \bar{u}+\beta y \bar{v} m^{2} \sigma-i f_{0} \sigma m \bar{v}_{y}=k N^{2}\left(-i k \bar{u}-\bar{v}_{y}\right)
\end{aligned}
$$


Eliminating $u$ from (7.13) and (7.14), an equation for the meridional velocity (amplitude part) is

$$
\begin{aligned}
& \sigma\left(N^{2}+f_{0}^{2}\right) \bar{v}_{y y}+2 i f_{0} \sigma m \beta y \bar{v}_{y} \\
& +\left[-\sigma\left(k^{2} N^{2}-m^{2} \sigma^{2}\right)-\beta^{2} y^{2} m^{2} \sigma+\beta k N^{2}+i f_{0} m \beta \sigma\right] \bar{v}=0
\end{aligned}
$$

This is a second order variable coefficient ordinary differential equation. Let $\bar{v}=G(y) Q(y)$, choose $G$ so that the coefficient of first derivative of $Q$ with respect to $y$ vanishes. Then we obtain

$$
G=e^{-\frac{i f_{\alpha} m \beta}{2\left(N^{2}+f_{\partial}^{2} \partial\right.} y^{2}}
$$

Then, an equation for $Q$ is

$$
Q^{\prime \prime}+\left[A-C y^{2}\right] Q=0
$$

where

$$
\begin{aligned}
& A=\frac{\beta k N^{2}}{\sigma\left(N^{2}+f_{0}^{2}\right)} \cdot \frac{\left(k^{2} N^{2}-m^{2} \sigma^{2}\right)}{N^{2}+f_{0}^{2}} \\
& C=\frac{N^{2} m^{2} \beta^{2}}{N^{2}+f^{2} \partial^{2}}
\end{aligned}
$$

Let $y=\lambda \eta$, where $\lambda$ is a meridional scale parameter

$$
\frac{\mathrm{d}^{2} \mathrm{Q}}{\mathrm{d \eta ^{2 }}}+\left[\lambda^{2} \mathrm{~A}-\lambda^{4} \mathrm{C} \eta^{2}\right] \mathrm{Q}=0
$$

This is an eigenvalue problem, when

$$
\begin{aligned}
& \lambda^{4} \mathrm{C}=1 \\
& \lambda^{2} \mathrm{~A}=2 \mathrm{j}+1
\end{aligned}
$$

$\mathrm{Q}$ has eigen solutions. So, the dispersion relation is

$$
\frac{\beta k N^{2}}{\sigma}-\left(k^{2} N^{2}-m^{2} \sigma^{2}\right)=(2 j+1) m \beta N
$$

and

$$
\lambda=\frac{\beta \mathrm{kN}^{2}}{\sigma} \sqrt{\frac{\mathrm{N}^{2}+\mathrm{f}_{0}^{2}}{\mathrm{Nm} \beta}}
$$


Let $\varphi_{j}(\eta)$ be the solution of

$$
\frac{d^{2} \varphi_{j}}{d \eta^{2}}+\left[(2 j+1)-\eta^{2}\right] \varphi_{j}=0
$$

Then, the our meridional velocity is

$$
v(x, y, z, t)=e^{i\left(\sigma t+k x+m z-\frac{f_{m} m}{2^{2} y^{2}} y^{2}\right.} \varphi_{j}\left(\frac{y}{\lambda}\right)
$$

or, in real form

$$
v=\varphi_{j}\left(\frac{y}{\lambda}\right) \cos \left[\sigma t+k x+m z-\frac{f_{0} m \beta}{2\left(N^{2}+f_{0}^{2}\right)} y^{2}+\Phi_{0}\right]
$$

\section{Discussion:}

\section{(a) Dispersion relation and group velocity}

The horizontal Coriolis parameter $f_{0}$ does not appear in dispersion relation (7.23) which suggests that the group velocity in the zonal and vertical directions has no differences from that of the conventional equatorial wave model. If we use the generalized equatorial wave model ( no traditional approximation is made) to explain the deep jets, the difficulty with the vertical downward group velocity being too small to provide deep jets energy from the upper ocean still exists. This result, combined with the scaling arguments in section 3 again suggests that some nonlinear effects may be involved. It is seen in the solution (7.26), that the y structure and $z$ structure are not totally separable, a parabolic phase contribution is in the phase. Although the effect of the horizontal Coriolis parameter is missing in the dispersion relation, its effect is in the structure of the physical quantities and needs to be studied. The following discussions address the role of the horizontal Coriolis parameter on the meridional structure of various physical quantities.

(b) Phase and modified depth 
Although the dispersion relation (7.23) does not involve $f_{0}$, the phase relation (7.25) does. Given that

$$
\begin{aligned}
& \Phi=\sigma t+k x+m z \frac{f_{0} m \beta}{2\left(N^{2}+f_{0}^{2}\right)} y^{2}+\Phi_{0} \\
& =\sigma t+k x+m\left(z \frac{\mu^{2}}{2 a\left(1+\mu^{2}\right)} y^{2}\right)+\Phi_{0}
\end{aligned}
$$

where $\mu=2 \Omega N$ denotes the ratio between the relative effect of the horizontal component of earth's rotation near equator and vertical stratification. In most of the ocean and atmosphere $\mu<<1$. Usually $\mu=0.1$ away from thermocline. We can see that although there is phase propagation in the y direction, it is not linear, which is different from the conventional wave. The phase speed in the y direction depends on the latitude. If we define $z_{b}=z-\frac{\mu^{2}}{2 a\left(1+\mu^{2}\right)} y^{2}$ as a modified depth, we can see that for the meridional velocity, the effect of the horizontal Coriolis parameter is completely involved in the modified depth. Fig.7.1 (a), (b) show the comparison of real depth and modified depth for different $\mu$. It is clear that for large $\mu$, the modification is very important, while for small $\mu$, the modification is not significant.

\section{(c) Meridional velocity structure}

Apart from a phase modification, the modal velocity structure of the meridional velocity structure is the same as the conventional structure except for an elongation or contraction factor $\left(1+\mu^{2}\right)^{\frac{1}{2}}$ of meridional scale depending on the ratio $\mu$. The elongation factor is graphed in Fig.7.2, which shows that when $\mu$ is small, this modification is very small.

For $\mathrm{N}^{2}>\mathrm{f}_{0}^{2}$

$$
v=\varphi_{j}\left(\frac{y}{\lambda}\right) \cos \left(\sigma t+k x+m z-\frac{f_{0} m \beta}{2 N^{2}} y^{2}\right)
$$

where 


$$
\lambda=\left(\frac{N}{m \beta}\right)^{\frac{1}{2}}
$$

A meridional scale is associated with the extra term in the phase,

$$
\frac{\mathrm{f}_{0} \mathrm{~m} \beta}{2 \mathrm{~N}^{2}} \mathrm{~L}_{\mathrm{y}}^{2}=\frac{\pi}{2}
$$

If parameters $f_{o}=2 \Omega, \beta=\frac{2 \Omega}{a}, m=\frac{2 \pi}{L_{z}}\left(L_{Z}\right.$ is vertical wavelength $)$ are inserted, then

$$
L_{y}=\frac{N}{\sqrt{2} 2 \Omega} \sqrt{a L_{z}}
$$

For $\mathrm{L}_{\mathrm{x}}=10,000 \mathrm{~km}, \mathrm{~L}_{\mathrm{z}}=100 \mathrm{~m}, \mathrm{a}=6.4 \times 10^{3} \mathrm{~km}, 2 \Omega=1.45 \times 10^{-4} \mathrm{rad} / \mathrm{s}$, and $\mathrm{N}=10^{-3}$ $\mathrm{rad} / \mathrm{s}$, then, $L_{y}=120 \mathrm{~km}$. Notice this scale is independent of frequency and meridional mode number if the vertical wavenumber is given. So if there are lots of meridional modes in the motion, this meridional length scale will still be the same.

$L_{y}$ is proportional to the Brunt Väisälä frequency, so if the vertical wavelength is much smaller than the variation scale of $\mathrm{N}$, then $\mathrm{L}_{\mathrm{y}}$ can be approximately viewed in a WKBJ sense as being larger in the upper ocean and smaller in the deeper ocean. For long vertical scale $L_{z}$ motion, $L_{y}$ is even larger.

\section{(d) Zonal velocity}

Knowing the meridional velocity, we can compute the zonal velocity from (7.14) and get

$$
\bar{u}=e^{i\left(\sigma t+k x+m z-\frac{f_{0} m \beta}{2\left(N^{2}+f_{0}^{2}\right)} y^{2}\right)}\left(u_{r}+i u_{i}\right)
$$

where

$$
\begin{aligned}
& u_{r}=\frac{f_{0} m N \beta y \varphi_{j}}{(k N+m \sigma)\left(N^{2}+f_{0}^{2}\right)}+\frac{f_{0} \sigma m}{k^{2} N^{2}-m^{2} \sigma^{2}} \sqrt{\frac{2 j N m \beta}{N^{2}+f_{0}^{2}}} \varphi_{j-1} \\
& u_{i}=\frac{N^{2} m \beta y \varphi_{j}}{(k N+m \sigma)\left(N^{2}+f_{0}^{2}\right)}+\frac{k N^{2}}{k^{2} N^{2}-m^{2} \sigma^{2}} \sqrt{\frac{2 j N m \beta}{N^{2}+f_{0}^{2}}} \varphi_{j-1}
\end{aligned}
$$

Using the aditional approximation is equivalent to setting $f_{0}=0$ in the above equations which result in exactly the same solution as that obtained in the conventional way. When 
$f_{0} \equiv 0$, the modified phase effect is missing and also $u_{r}=0$. In the real ocean $\frac{f_{0}}{N} \ll 1$, so the contribution of $u_{r}$ to the amplitude is very small. Since the phase modification effect has been discussed in (b), only comparisons of amplitude for modified and unmodified waves under different set of parameters are discussed hereafter.

We choose the following values of physical parameters as our standard set and make the comparison when one of them is changed: Meridional mode number $j=3$; vertical wavelength $L_{z}=600 \mathrm{~m}$; Brunt Väisälä frequency $N=1.7 \times 10^{-3} \mathrm{rad} / \mathrm{s}$; zonal wavelength $\mathrm{L}_{\mathrm{X}}=1000 \mathrm{~km}$. The corresponding frequency can be computed from the dispersion relation (7.23). Fig.7.3 (a),(b) show the comparison when meridional mode number is changed (a) for $j=1$ (b) for $j=5$. The solid curve shows the amplitude $\sqrt{u_{p}^{2}+u_{i}^{2}}$ of the meridional structure for the modified wave and the dashed curve for the unmodified wave. It is seen that the modification is very small. Fig.7.4 (a),(b) are the comparisons of amplitude under a standard parameter set except that the vertical wavelength is changed: (a) for $\mathrm{L}_{\mathbf{z}}=4000 \mathrm{~m}$ and (b) for $L_{z}=100 \mathrm{~m}$. The difference is insignificant. Fig.7.5 (a),(b) show the effect when zonal wavelength changes: (a) for $L_{x}=10^{7} \mathrm{~m}$ (b) for $L_{x}=10^{5} \mathrm{~m}$. All these plots show that the modifications are very small. Fig. 7.6 (a), (b) show the effect of vertical stratification. (a) for $\mathrm{N}=10^{-3} \mathrm{rad} / \mathrm{s}$. (b) for $\mathrm{N}=0.2 \times 10^{-3} \mathrm{rad} / \mathrm{s}$. It is seen that for small stratification, the modification is very large. Fig. 7.7 (a), (b) show the effect on different types of waves: (a) $\sigma=1.4 \times 10^{-7} \mathrm{rad} / \mathrm{s}$ for lower frequency wave and (b) $\sigma=5.1 \times 10^{-6}$ for higher frequency wave under same parameter set. It is seen that the modification is small for both higher and lower frequency waves.

A short conclusion is that the effect of horizontal Coriolis parameter on linear equatorial trapped waves is very small for a variety of physical parameters. It is the strong stratification which prevents the modification from becoming important. Although the phase $\tan ^{-1}\left(\frac{u_{r}}{u_{i}}\right)$ modification has not been presented in plots, it can be seen that this modification is small since $\left|u_{h}\right|<<\left|u_{i}\right|$, except at very few locations. 
(e) Vertical Velocity

From (7.11), the vertical velocity can be easily obtained as

$$
\bar{w}=e^{i\left(\sigma t+k x+m z \frac{f_{0} m \beta}{2\left(N^{2}+f_{0}^{2}\right)} y^{2}\right)}\left(w_{r}+i w_{i}\right)
$$

where

$$
\begin{aligned}
& w_{r}=\frac{m \sigma f_{0} \beta y \varphi_{j}}{(k N+m \sigma)\left(N^{2}+f_{0}^{2}\right)}-\frac{f_{0} \sigma k}{k^{2} N^{2}-m^{2} \sigma^{2}} \sqrt{\frac{2 j N m \beta}{N^{2}+f_{0}^{2}}} \varphi_{j-1} \\
& w_{i}=\frac{m \sigma N \beta y \varphi_{j}}{(k N+m \sigma)\left(N^{2}+f_{0}^{2}\right)}-\frac{m \sigma^{2}}{k^{2} N^{2}-m^{2} \sigma^{2}} \sqrt{\frac{2 j N m \beta}{N^{2}+f_{0}^{2}}} \varphi_{j-1}
\end{aligned}
$$

Fig.7.8 shows the modification on the amplitude of the meridional structure of the vertical velocity. The modification is small. We will not show the effect on density and pressure perturbations since this effect is also small under real stratification conditions.

(f) Validity of the hydrostatic relation and the relative magnitude of the Coriolis acceleration terms in the zonal momentum equation

The vertical momentum equation (7.3) is not fully hydrostatic. The goodness of hydrostatic relation can be measured by the smallness of the following ratio,

$$
\gamma=-\frac{f_{0} u}{\frac{\rho}{\rho_{0}} g}
$$

Its meridional distribution can be obtained by using (7.5)

$$
i \sigma \rho=-w \rho_{0 z}
$$

so

$$
\left.\rho=\frac{\rho_{0} N^{2}}{g \sigma} e^{i(\sigma t+k x+m z} \frac{f_{0} m \beta}{2\left(N^{2}+f_{0}^{2}\right)} y^{2}\right)\left(-w_{i}+i w_{r}\right)
$$

Then the amplitude of the above ratio is

$$
\gamma=\frac{\mathrm{f}_{0} \sigma|\mathrm{u}|}{\mathrm{N}^{2}|\mathrm{w}|}
$$

Fig.7.9 (a), (b) show the size of $\gamma$ for different vertical wavelengths, (a) for $L_{z}=4000 \mathrm{~m}$,

(b) for $\mathrm{L}_{\mathrm{z}}=600 \mathrm{~m}$. It is seen that $\gamma$ is always less than one over all latitudes, and is generally 
small. In a few very localized places, it may close to 1 . Fig.7.10 (a), (b) is similar to Fig.7.9. Except for higher frequency waves, it is seen that the large $\gamma$ peaks are even more localized. So the effect of the horizontal Coriolis parameter on the hydrostatic relation is stronger at lower frequencies and at larger vertical wavelengths. However, the effect is small except at a few very localized locations.

The relative size of the Coriolis acceleration terms in the zonal momentum equation is of interest because the vertical Coriolis parameter approaches zero when the equator is approached. We plotted the ratio $\frac{\beta y v}{f_{0} w}$ in the following graphs. Fig. 7.11 (a), (b) show the ratio for low frequency waves under (a) $\mathrm{L}_{\mathrm{z}}=600 \mathrm{~m}$, (b) $\mathrm{L}_{\mathrm{z}}=4000 \mathrm{~m}$. The ratio is everywhere very large except very close to equator (about $20 \mathrm{~km}$ ). Fig. 7.12 (a), (b) are for higher frequency waves (a) $L_{z}=600 \mathrm{~m}$, (b) $\mathrm{L}_{\mathrm{Z}}=4000 \mathrm{~m}$. It is seen that the $f_{\mathrm{O}}$ term is important for a certain range of latitudes, especially for long vertical wavelength waves. A preliminary conclusion is that for linear equatorial waves, the effect of the horizontal Coriolis parameter is more important for high frequency waves in the zonal equation and for low frequency waves in the vertical momentum equation. Although from the relative size of terms in momentum equations, we cannot neglect the effect of the horizontal Coriolis parameter, from the solutions we get, under strong stratification we can use the unmodified wave solution when the horizontal Coriolis parameter is being neglected. For the case, $N \equiv 0$, (7.18) and (7.19) reduced to $A=\frac{m^{2}}{f_{0}^{2}} \sigma^{2}, C=0$. Equation (7.17) becomes $Q^{\prime \prime}+\frac{m^{2}}{f_{0}^{2}} \sigma^{2} Q=0$, and its solutions are $Q=e^{i \frac{m \sigma}{f_{o}}}$. So, meridional velocity is $v=e^{i\left(\sigma t+k x+m z+\frac{m \sigma}{f_{n}} y \frac{m \beta y^{2}}{2 f_{0}}\right)}$. This problem has been solved by Stern (1963) although his solution was expressed in a different form. The meridional scale associated with the extra term in the phase is $L_{y}=\sqrt{\frac{a L_{2}}{2}}$ which is of the right order on the boundary where the two 
Coriolis terms in the zonal momentum equation are of the same importance for motions of vertical scale, $L_{z}$.(Section 4)

For small vertical wavelength motions, the friction effect becomes important. The difficulty in finding a suitable parameterization prevents us from having a good understanding of the frictional effect. We temporarily assume that the friction term is of a diffusive type with an eddy viscosity.

For simplicity, we study the vertical momentum friction effect with zonal variation neglected. After the traditional approximation is used, our starting equations become

$$
\begin{aligned}
& u_{t}-\beta y v=A_{v} u_{z z} \\
& v_{t}+\beta y u=-\frac{1}{\rho_{0}} P_{y}+A_{v} v_{z z} \\
& 0=-\frac{1}{\rho_{0}} P_{z}+g \frac{\rho}{\rho_{0}} \\
& \rho_{t}+w \frac{\partial \rho_{0}}{\partial z}=0
\end{aligned}
$$

Still looking for a wave type solution as in (7.9), a dispersion relation can be obtained as

$$
\sigma m-i A_{v} m^{3}=\sqrt{(2 j+1) N m \beta}
$$

Let $\sigma=\sigma_{\mathrm{r}}+\mathrm{i} \sigma_{\mathrm{i}}$, we see that the decaying e-folding scale is $\sigma_{\mathrm{i}}=\mathrm{A}_{\mathrm{v}} \mathrm{m}^{3}$ which increases when the vertical wavelength decreases. The increasing factor goes as the cube of the vertical wavenumber.

The above analysis can be done in a similar way for equatorial Kelvin waves. This section gives us confidence that for a linear inviscid equatorial wave system, the horizontal Coriolis parameter effect can be neglected due to the presence in reality of stratification. However, we cannot go one step further to believe that the effect is also negligible for all other equatorial systems. The following section is to examine the effect of momentum friction and density diffusion on the role of the horizontal Coriolis parameter. 


\section{Section 8. Effect of momentum friction and density diffusion}

One of the extreme cases of the general governing equation derived in Section 3 is that when $R \rightarrow 0$, the nonlinearity can be ignored. The effect of advective nonlinearity has been discussed in Section 5 and Section 6. In dimensional form, the linear continuously stratified viscous model consists of the following

$$
\begin{aligned}
& -\beta y v+f_{0} w=A_{v} \frac{\partial^{2} u}{\partial z^{2}} \\
& \beta y u=-\frac{1}{\rho_{0}} \frac{\partial P}{\partial y}+A_{v} \frac{\partial^{2} v}{\partial z^{2}} \\
& -f_{0} u=-\frac{1}{\rho_{0}} \frac{\partial P}{\partial z}-\frac{\rho}{\rho_{o}} g \\
& \frac{\partial v}{\partial y}+\frac{\partial w}{\partial z}=0 \\
& w \frac{\partial \rho_{o}}{\partial z}=K_{v} \frac{\partial^{2} \rho}{\partial z^{2}}
\end{aligned}
$$

where $\mathrm{f}_{0}$ denotes the horizontal Coriolis parameter, which is $2 \Omega$ at equator. McPhaden (1981) examined this system neglecting terms associated with $\mathrm{f}_{0}$. He tested the sensitivity of his results to different turbulence parameterizations. One is characterized by Newtonian cooling, the other has biharmonic form of friction. He assumed the dissipation was of these forms mainly because in these ways the resulting problems were spatially separable and so analytically tractable. We will still use equation set (8.1) - (8.5) to examine the role of the horizontal Coriolis parameter since the problem is already spatially nonseparable. If we assume our solution is of type,

$$
\left(\begin{array}{c}
u \\
v \\
w
\end{array}\right)=\left(\begin{array}{c}
\bar{u} \\
\bar{v} \\
\bar{w}
\end{array}\right) e^{i m z}
$$

then, the amplitude of meridional velocity satisfies 


$$
\left(\frac{f_{0}}{m^{2} A}-\frac{N^{2} A}{K_{v} m^{2}}\right) \bar{v}_{y y}+\frac{2 f_{0} \beta y}{m} \bar{v}_{y}+\left(\frac{i f_{0} \beta}{m}-\beta^{2} y^{2}-A^{2} m^{4}\right) \bar{v}=0
$$

Let

$$
\bar{v}(y)=e^{-\frac{f_{0} \beta y^{2}}{2 m\left(\frac{f_{0}^{2}}{m^{2}}-\frac{N^{2} A}{K u m^{2}}\right)} Q(y)}
$$

then $Q(y)$ satisfies

$$
\left(1+\gamma^{2}\right) Q^{\prime \prime}+\left(\frac{m}{a}(i-1)-\frac{A^{2}}{4 \Omega^{2}} m^{6}-\frac{m^{2}}{a^{2}} \frac{2+\gamma^{2}}{1+\gamma^{2}} y^{2}\right) Q=0
$$

where

$$
\gamma^{2}=\frac{N^{2} P_{r}}{f_{0}^{2}}
$$

and $\quad P_{r}=\frac{A}{K_{v}}$ is the Prandtl number. Now (8.8) becomes

$$
\bar{v}(y)=e^{-\frac{m y^{2}}{2 a\left(1+\gamma^{2}\right)}} Q(y)
$$

It is seen that the importance of the horizontal Coriolis parameter is characterized by the nondimensional parameter $\gamma$. If $\mathrm{N}$ is ten times larger than $\mathrm{f}_{0}$, as the usual case, then the horizontal Coriolis parameter becomes important only when $\mathrm{K}_{\mathrm{v}}$ is a hundred times larger than $A_{v}$. The estimate of eddy viscosity is far from satisfactory, especially in the deep ocean. Santiago-Mandujana and Firing(1990) created a summary table (Table 1) of the eddy viscosity coefficient estimates $A_{V}$ from upper equatorial ocean observations, but the magnitude of which in various observations differs by at least an order of magnitude. We are not going to study the model in detail. The conclusion we get is that the turbulent diffusion may affect the importance of the horizontal Coriolis parameter. 


\section{Section 9. Concluding Remarks}

Two topics have been discussed in this thesis. A nonlinear equatorial deep jets model and the role of the horizontal Coriolis parameter. Both these topics are very difficult to study and have been discussed in the literature for quite some time.

It is seen that the free, inertial model is a qualitatively consistent model capable of describing many features of the equatorial deep jets. The meridional motion is confined to the equator. The zonal velocity consists of a background of planetary zonal velocity and the small vertical scale equatorial deep jets signal. The $\beta$-effect mainly determines the meridional trapping of the jets. The magnitude of the density anomaly is what mainly determines the vertical structure. It confirms the possibility of the interpretation of equatorial deep jets as free, inertial motion below the thermocline. However, this model still has several shortcomings. Since it involves several free constants which can not be determined, the accurate comparison of vertical and horizontal jet scales of the model with observations becomes very difficult. The model does not include momentum and density dissipation, so the interpretation in the very deep ocean becomes quite qualitative. The neglect of zonal dependence avoids the discussion of deep jets near eastern and western boundaries. A scale matching of one mode of the model with observation reveals that the latitudinal trapping scale of the model is five times smaller than that observed in the ocean. If more modes are involved and better choice of the arbitrary functions is made, this matching may become better.

Given the small vertical scale of the phenomenon, it is hard to do numerical computations to resolve the jets. More field observations to measure the meridional velocity as well as the zonal velocity are needed. Knowing the correlation of these two components of the horizontal velocity may be of importance since it tells us the connection between the meridional motion and the zonal velocity. A more systematic observational confirmation of 
the planetary zonal velocity is needed. Theoretically, more discussion of the potential zonal vorticity equation is needed. It is of great interest to solve and interpret its solution when different choices of the arbitrary functions are made. It is difficult to simulate a free, inertial motion by numerical computation since its generation mechanism is unknown. One possible way of doing numerical computational analysis on equatorial deep jets is to develop a numerical model to solve the motion on a meridional plane with realistic forcing and background density distribution. Then, a statistical average can be made to see if this average compares well with the analytical solution proposed in Section 6.

The second topic discussed in the thesis is the role of the horizontal Coriolis parameter. A general conclusion is still missing. In the simplified models used in the previous several sections. It is found that this parameter is not important in linear inviscid stratified equatorial waves. The role may be enhanced if momentum friction and density dissipation effects are included and the ratio of one to the other is small. In the nonlinear equatorial dynamics, it is seen that the horizontal Coriolis parameter-related term in the zonal momentum equation acts as an important contributor to the conservation of angular momentum. It provides a vertical background zonal velocity shear which is qualitatively confirmed by observations. The effect of the horizontal Coriolis parameter in the vertical momentum equation is belittled by the presence of density anomalies associated with the fluid motion. It is suggested that these two terms be included in the future numerical models dealing with large vertical scale motions. Most existing models mainly study the fluid motion in the upper ocean. Future three dimensional models involving thermohaline circulation may find some effects from this parameter. 


\section{Acknowledgement}

I would like to thank my thesis advisor Prof. Carl Wunsch for his many encouragements, stimulating discussions, criticism and fatherly advices. He painstakingly read many drafts and carefully cleared and corrected hundreds of unclear places and grammartical mistakes. Without his guidance and support, this thesis would not be possible. The funding was provided by National Aeronautics and Space Administration under grant NAGW - 1048.

I also thank many Joint Program professors and students for many discussions with them from which I benefitted greatly, in particular, to Prof. Nelson Hogg, Prof. Xin Huang, James Gunson, Kirill Pankratov and Alison Macdonald. Dorothy Frank typed the first draft. Jérôme Benveniste gave much assistance in using Macintosh.

Finally, I would like to express my deepest gratitude to my wife, Peiying, for her understanding and encouragement. 


\section{References}

Aldridge, K.D., 1967. An experimental study of axisymmetric inertial oscillations. Ph. D. Thesis, Geol. MIT.

Bjerknes, V., J. Bjerknes, H. Solberg and T. Bergeron, 1933. Physikalische Hydrodynamik. Mit Anwendung auf die Dynamische Meterologie. Springer, Berlin, 797pp.

Bretherton, F.P., 1964. Low frequency oscillations trapped near the equator. Tellus, 16, 181-185.

Charney, J. G., 1960. Non-linear theory of a wind-driven homogeneous layer near the equator. Deep-Sea Research, 6, 303-310.

Dobryshman, E. M., 1980. Dynamics of equatorial atmosphere, [in Russian] Gidrometeoizdat, Leningrad.

Dobryshman, E. M., 1987. Linear wave theory in low-latitude atmosphere. Meteorologiya $i$ Gidrologiya [Soviet Meteorology and Hydrology, No.1, 14-25.

Dobryshman, E. M., 1988. Quasi-three-dimensional modons in the low-latitude troposphere, Meterologiya i Gidrologiya, No.1, 5-16.

Eckart, C., 1960. Hydrodynamics of Oceans and Atmosphere, Pergamon Press, Macmillan, New York, 290pp.

Eriksen, C. C., 1981. Deep currents and their interpretation as equatorial waves in the Western Pacific Ocean. J. Phys. Oceanogr., 11, 48-70.

Eriksen, C. C., 1982. Geostrophic equatorial deep jets. J. Marine Res., Supplement to 40, $143-157$.

Firing, E., 1987. Deep zonal currents in the central equatorial Pacific. J. Marine Res., 45, 791-812.

Fofonoff, N. P., and R. B. Montgomery, 1955. The equatorial undercurrent in the light of the vorticity equation. Tellus, 7, 518-521. 
Gent, P., and J. R. Luyten, 1985. How much energy propagates vertically in the equatorial ocean? J. Phys. Oceanog., 15, 997-1007.

Grimshaw, R., 1975. Internal gravity waves: critical layer absorption in a rotating fluid. $J$. Fluid Mech., 70, 287-304.

Hayes, S. P., and H. B. Milburn, 1980. On the vertical structure of velocity in the Eastern Equatorial Pacific. J. Phys. Oceangr., 10, 633-635.

Hendershott, M. C., 1981. Long Waves and Ocean Tides. Ch.10, pp. 292-341, in Evolution of Physical Oceanography, B.A. Warren and C. Wunsch (Eds.), MIT Press.

Israeli, M., 1972. On trapped modes of rotating fluids in spherical shells. Studies in Applied Mathematics., 51, 219-237.

Joyce, M.T., 1988. On the hydrostatic balance and equatorial geostrophy. Deep-Sea Research, 35, 1255-1257.

Leetmaa, A. and P. F. Spain, 1981. Results from a velocity transect along the equator from $125^{\circ}$ to $159^{\circ} \mathrm{W}$. J. Phys. Oceanogr., 11, 1030-1033.

Long, R. R., 1958. Tractable models of steady-state stratified flow with shear. Quart. J. Roy. Meteor. Soc., 84, 159-161.

Luyten, J. R., and J. C. Swallow, 1976. Equatorial undercurrent. Deep-Sea Research, 23, $1005-1007$.

Matsuno, T., 1966. Quasi-geostrophic motions in the equatorial area. Journal of the Meteorological Society of Japan, series 2, 44, 25-43.

McCreary, J. P., 1984. Equatorial beams. J. Marine Res., 42, 395-430.

McCreary, J. P. and R. Lukas, 1986. The response of the equatorial ocean to a moving wind field. J. Geophys. Res., 91, 11691-11705.

McPhaden, M. J., 1981. Continuously stratified models of the steady-state equatorial ocean. J. Phys. Oceanogr., 11, 337-354. 
McPhaden, M. J., J. A. Proehl and L. M. Rothstein, 1986. The interaction of equatorial Kelvin waves with realistically sheared zonal currents. J. Phys. Oceanogr., 16, 1499-1515.

Miles, J.W., 1974. On Laplace's tidal equations. J. of Fluid Mech., 66, 241-260.

Moore, D.W., and S.G.H. Philander, 1977. Modeling of the tropical oceanic circulation. The Sea: Ideas and observations on progress in the study of the Sears, Vol. 6, E.D, Goldberg, I.N. McCare, J.J. O'Brien, and J.H. Steele, eds., John Wiley, Interscience, New York, pp. 319-361.

Munk, W. and N. Phillips, 1968. Coherence and band structure of inertial motion in the sea. Reviews of Geophysics, 6, 447-472.

Pedlosky, J., 1987. An inertial theory of the equatorial undercurrent. J. Phys. Oceangr., 17, 1978-1985.

Philander, S. G. H. and R. C. Pacanowski, 1981. Response of equatorial oceans to periodic forcing. J. Geophys. Res., 86, 1903-1916.

Philander, S. G. H. and R. C. Pacanowski, 1984. Simulation of the seasonal cycle in the tropical Atlantic Ocean. Geophys. Res. Lett., 11, 802-804.

Phillips, N.A., 1966. The equations of motion for a shallow rotating atmosphere and the 'traditional approximation'. J. Atmos. Sci., 23, 626-628.

Phillips, N.A., 1968. Reply [to Veronis (1968)]. J. Atmos. Sci., 25, 1155-1157.

Ponte, R. M., 1988a. Observations and Modelling of deep equatorial currents in the central Pacific. Ph. D. dissertation, Massachusetts Institute of Technology and Woods Hole Oceanographic Institution, $181 \mathrm{pp}$.

Ponte, R. M., 1988b. Equatorial Kelvin waves embedded in mean flow, with application to the deep jets. J. of Geophys. Res., 91, 11691-11705.

Ponte, R. M., 1989. A simple model for deep equatorial zonal currents forced at lateral boundaries. J. Phys. Oceanogr., 19, 1881-1891. 
Ponte, R. M. and J. Luyten., 1989. Analysis and interpretation of deep equatorial currents in the central Pacific., J. Phys. Oceanogr., 18, 1025-1038.

Ponte, R. M. and J. Luyten, 1990. Deep velocity measurements in the western equatorial Indian Ocean. J. Phys. Oceanogr., 20, 44-52.

Ponte, R. M., J. Luyten and P. L. Richardson, 1990. Equatorial deep jets in the Atlantic Ocean. Deep-Sea Research, 37, 711-713.

Proudman, J. 1942. On Laplace's differential equations for the tides, Proc. Roy. Soc., A179, 261-288.

Rothstein, L. M., D. W. Moore and J. P. McCreary, 1985. Interior reflections of a periodically forced equatorial Kelvin wave. J. Phys. Oceanogr., 15, 985-996.

Rothstein, L. M., M. J. McPhaden and J. A. Proehl, 1988. Wind forced wave-mean flow interactions in the equatorial waveguide. Part I: The Kelvin wave. J. Phys. Oceangr., 18, 1435-1447.

Santiago-Mandujano, F. and Firing E., 1990. Mixed-layer shear generated by wind stress in the Central Equatorial Pacific, J. Phys. Oceanog., 20, 1576-1582.

Solberg, H., 1928. Integrationen der atmospharischen storungsgleichungen. Erster Teil: Wellenbewegungen in rotierenden, inkompressiblen Flussigkeitsschichten. Geofyiske Publikasjoner, 5-9, 120pp.

Stern, M.E., 1963. Trapping of low frequency oscillations in an equatorial "boundary layer". Tellus, 15, 246-250.

Stewartson, K. and Rickard. J.A., 1969. Pathological oscillations of rotating fluid. J. Fluid Mech., 35, 759-773.

Stommel, H., 1960. Wind drift near the equator. Deep-Sea Research, 6, 208-302.

Veronis, G., 1968. Comments on Phillips (1966). Journal of the Atmospheric Sciences, 25, 1154-1155.

Wunsch, C., 1977. Response of an equatorial ocean to a periodic monsoon. J. Phys. Oceangr., 7, 497-511. 
Wunsch, C., and A. E.Gill, 1976. Observations of equatorially trapped waves in Pacific sea level variations. Deep-Sea Res., 23, 371-390.

Yih, C. -S., 1960. Exact solutions for steady two-dimensional flow of a stratified fluid. $J$. Fluid Mech., 9, 161-174. 

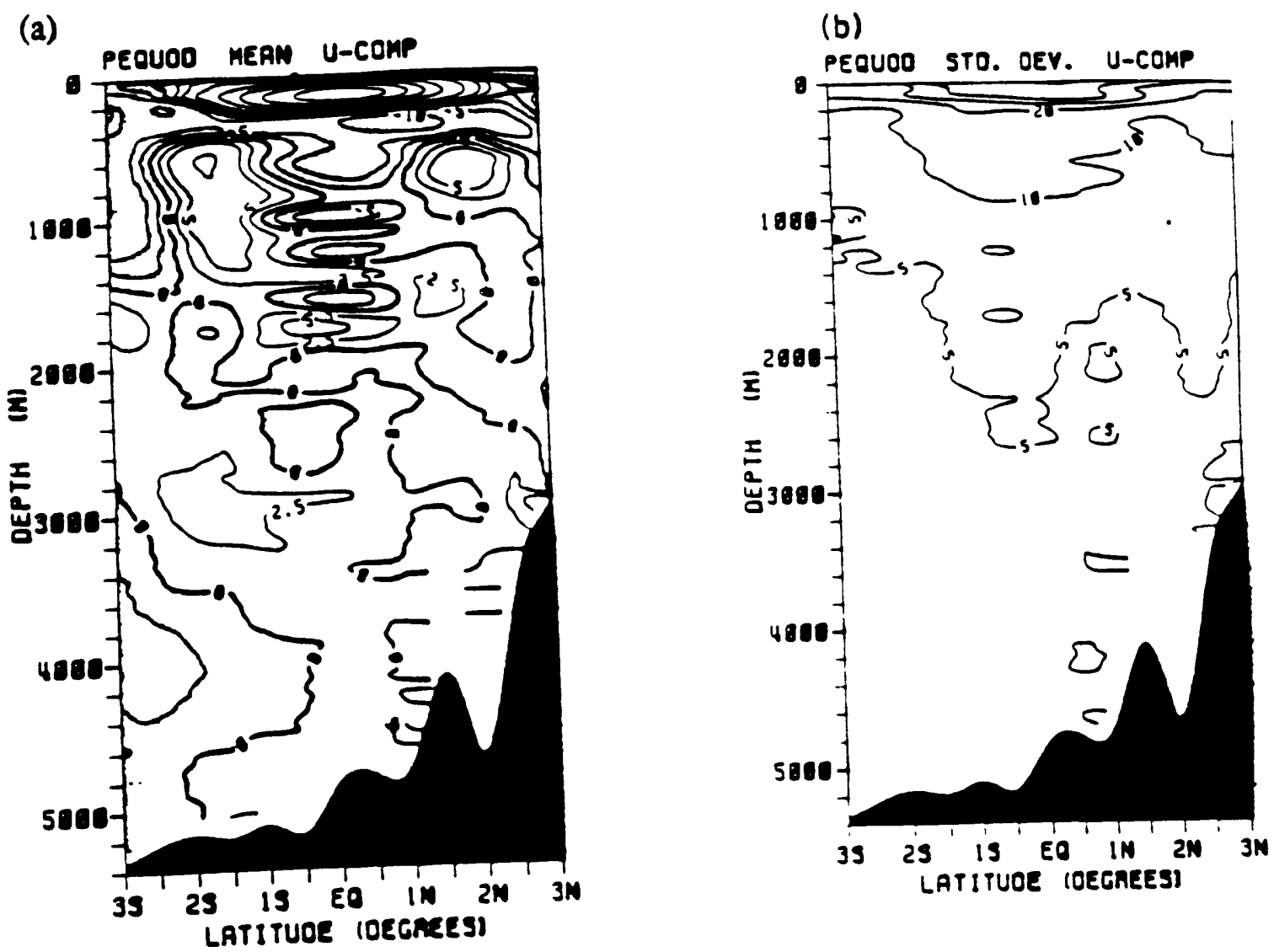

Fig.1.1. (a) Zonal current component along $1590 \mathrm{~W}$ for the period March 1982 - June 1983. Shown is the mean based on 21 cruises. Westward flow is shaded. (b) Standard deviation. (from Firing, 1987) 


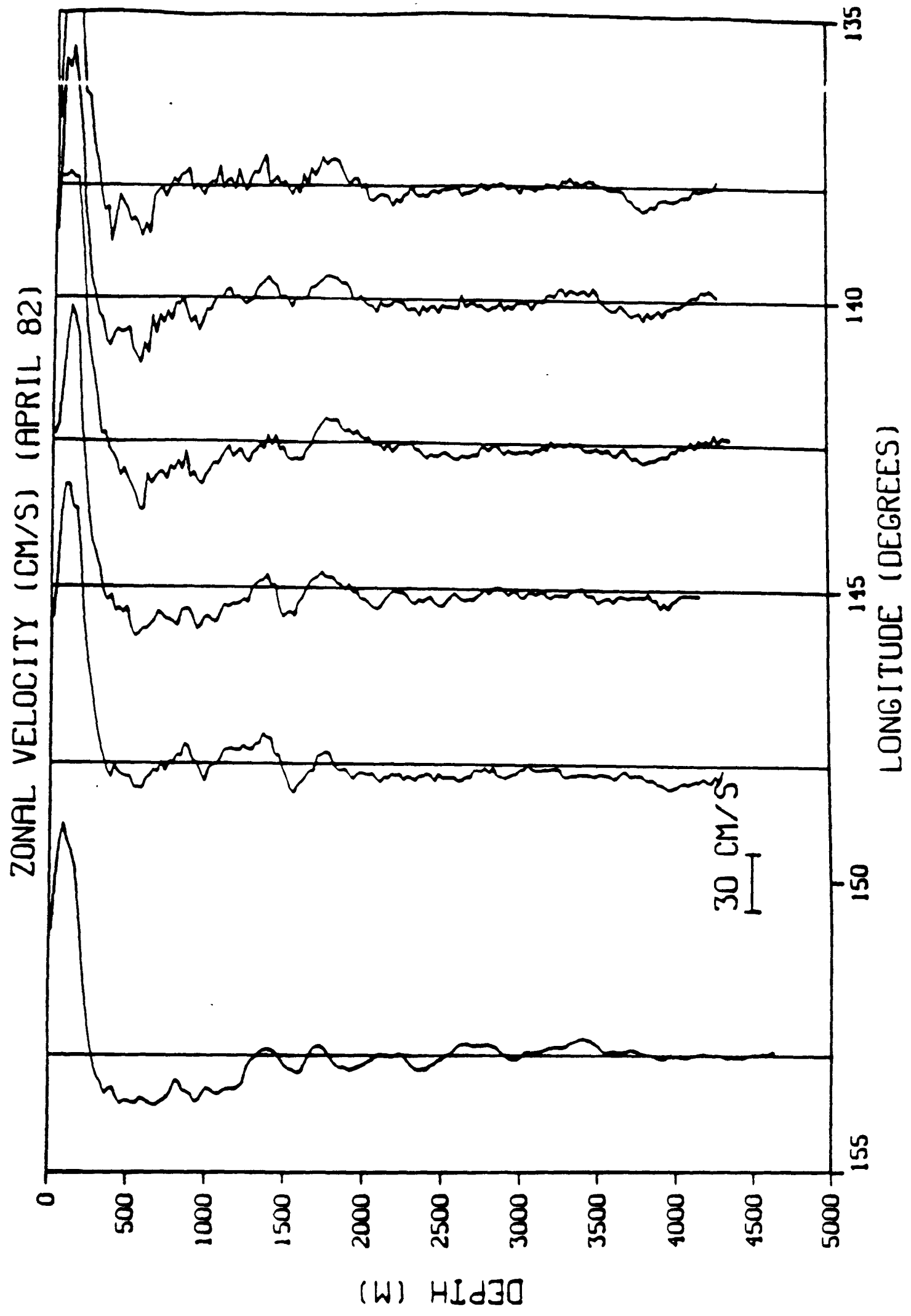

Fig.1.2. Zonal velocity along a longitudinal section at the equator. (from Ponte and Luyten, 1989) 


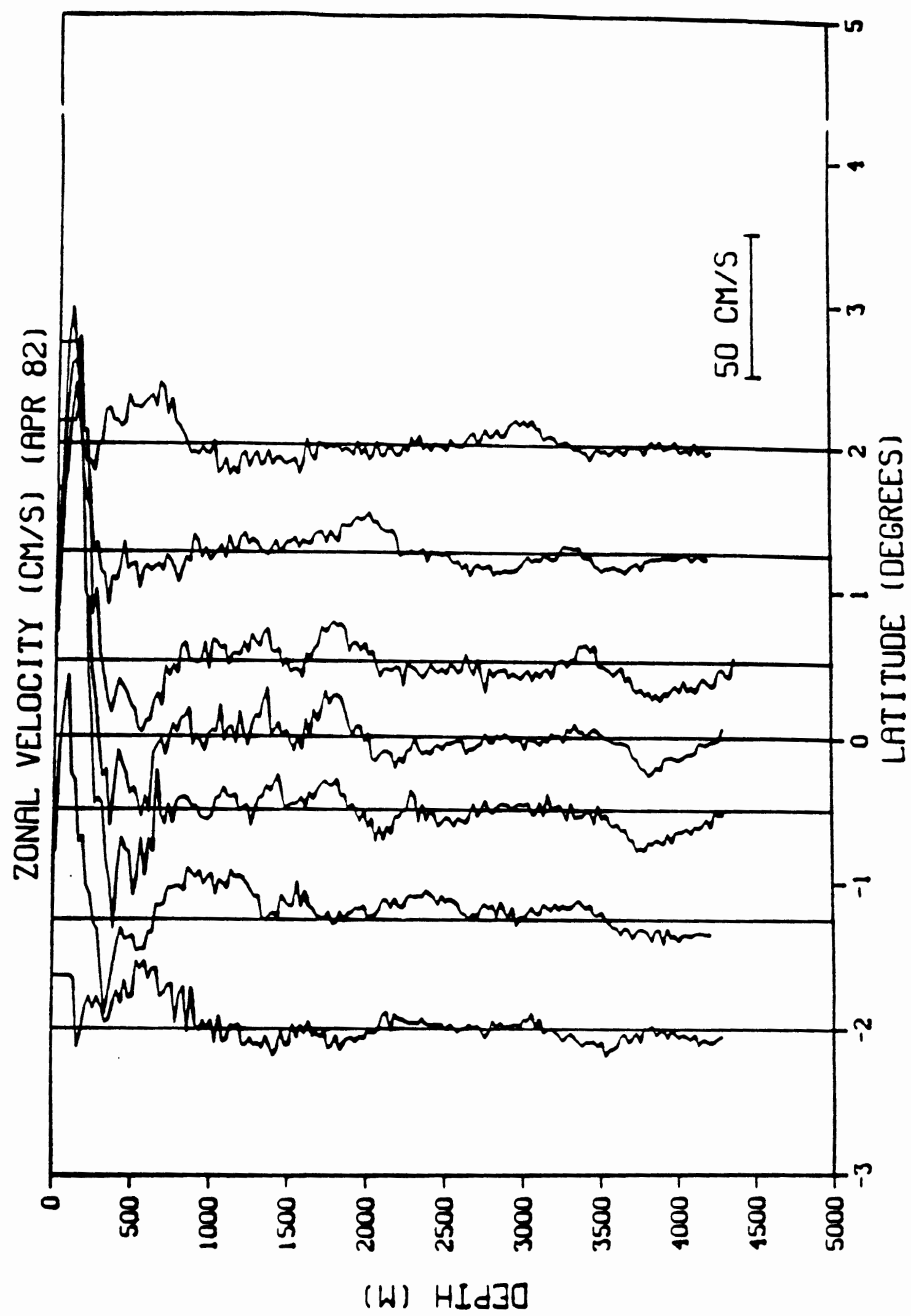

Fig.1.3. Zonal velocity along a lautudinal section at $138^{\circ} \mathrm{W}$. (from Ponte and Luyten, 1989) 


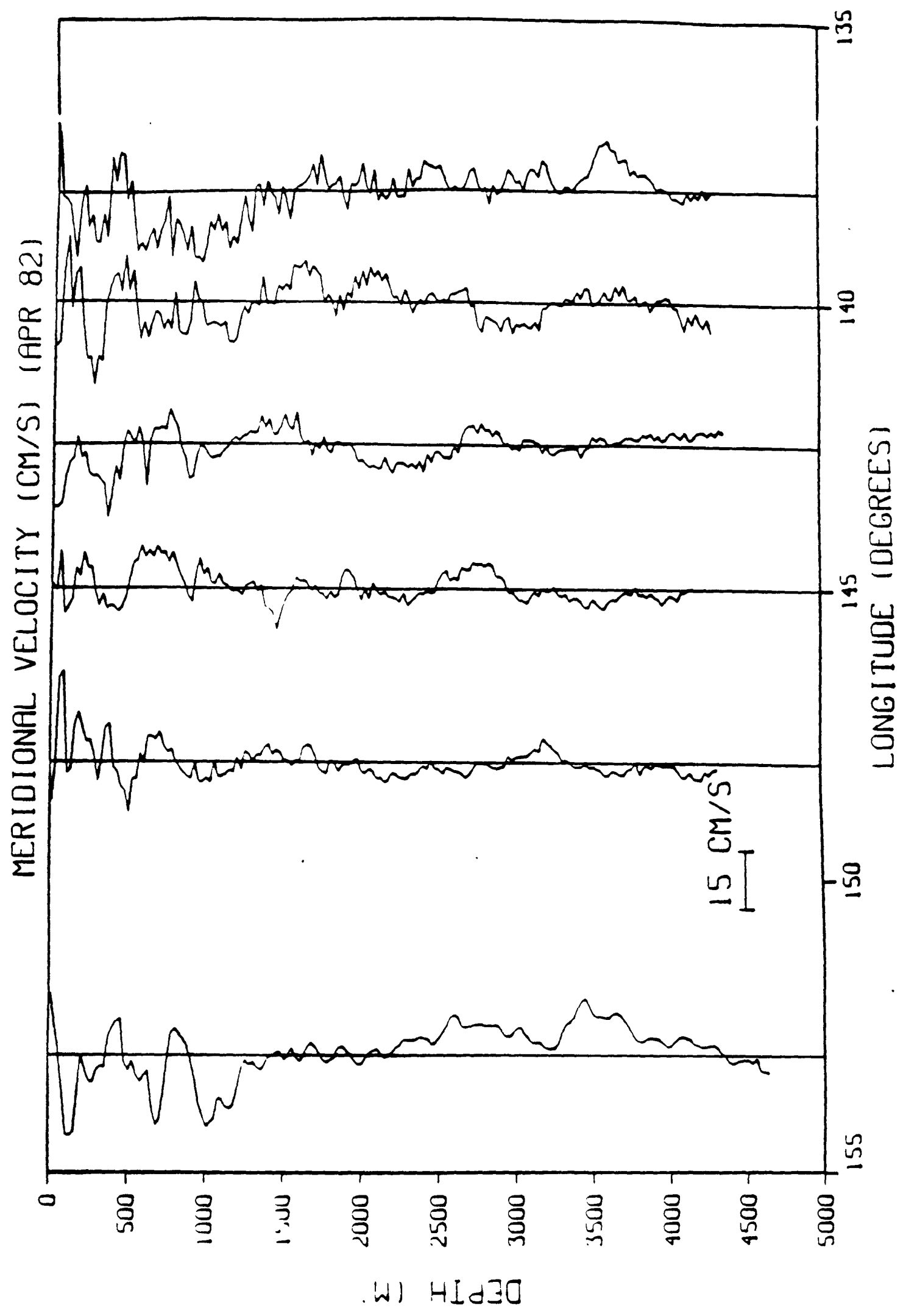

Fig.1.4. Meridional velocity along a longitudinal section at the equator. (from Ponte, 1988a) 


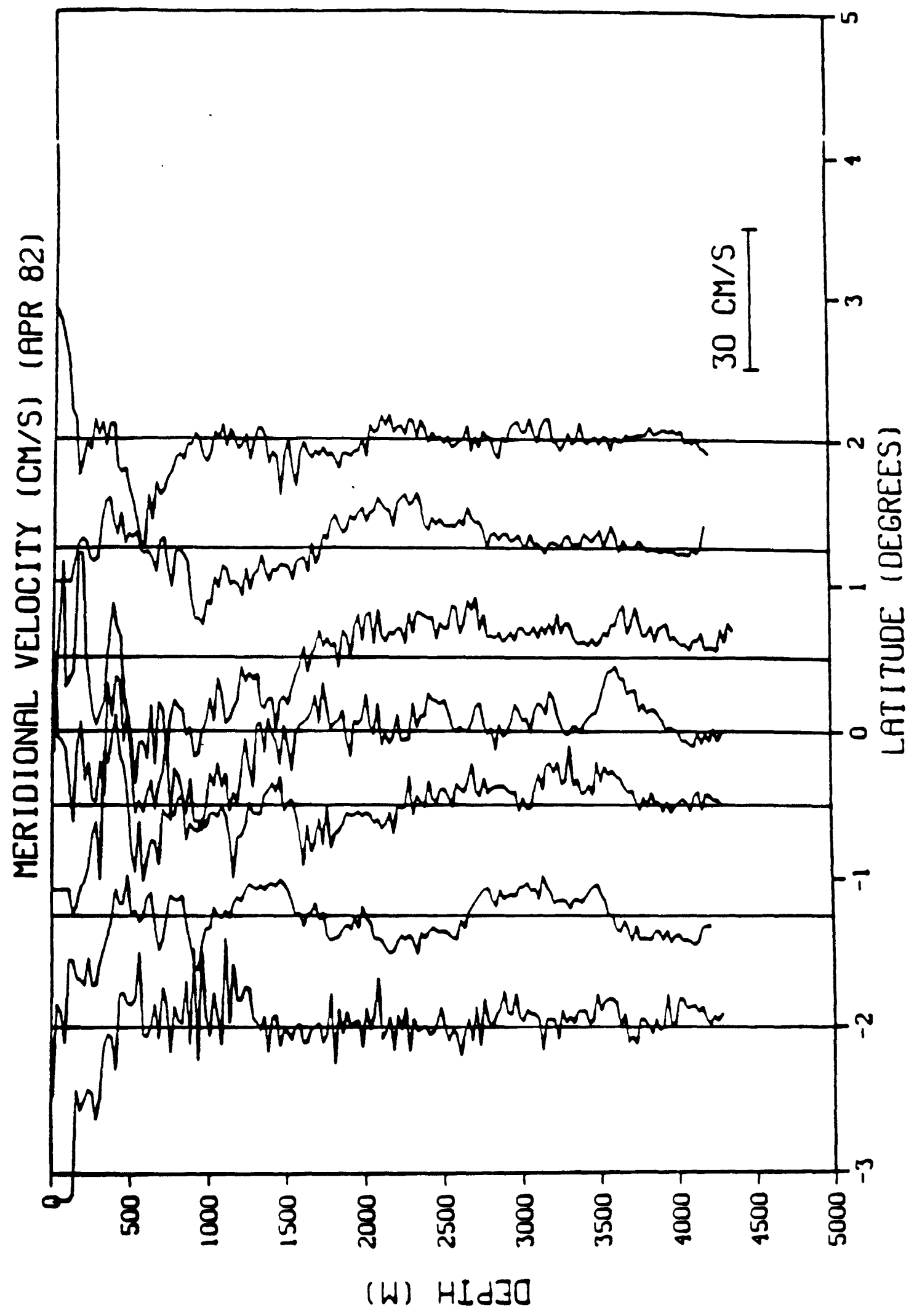

Fig.1.5. Meridional velocity along a latitudinal section at $1380 \mathrm{~W}$. (from Ponte, 1988a) 


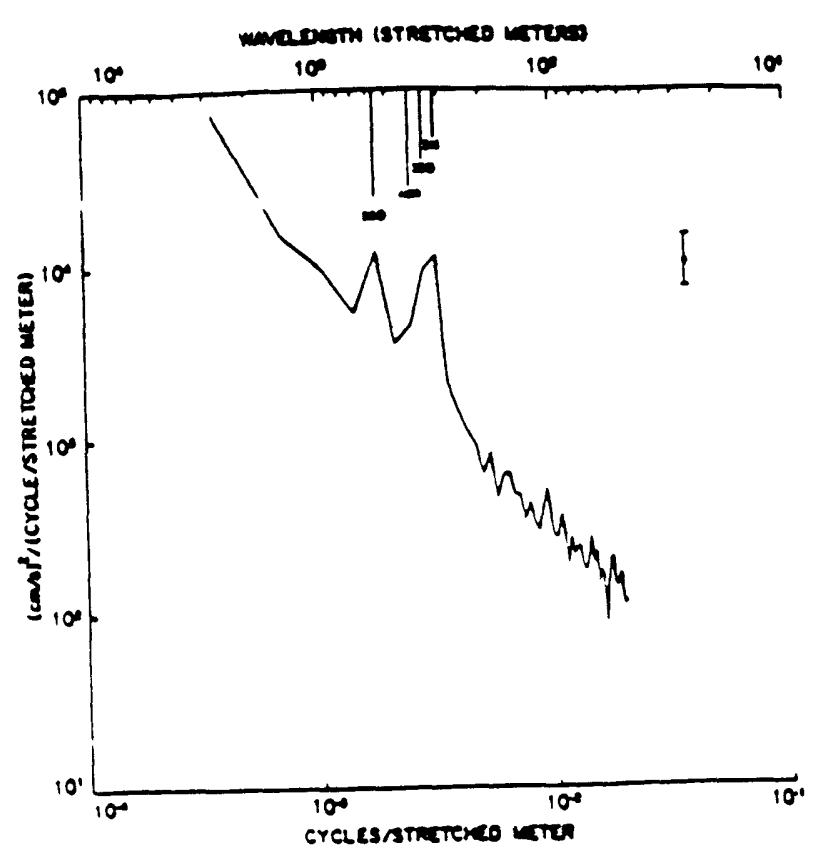

Fig.1.6. Zonal velocity power spectrum with $95 \%$ confidence error bar (from Ponte and Luyten, 1989). Notice in this figure, the length unit used is stretched meters instead of usual meters. 


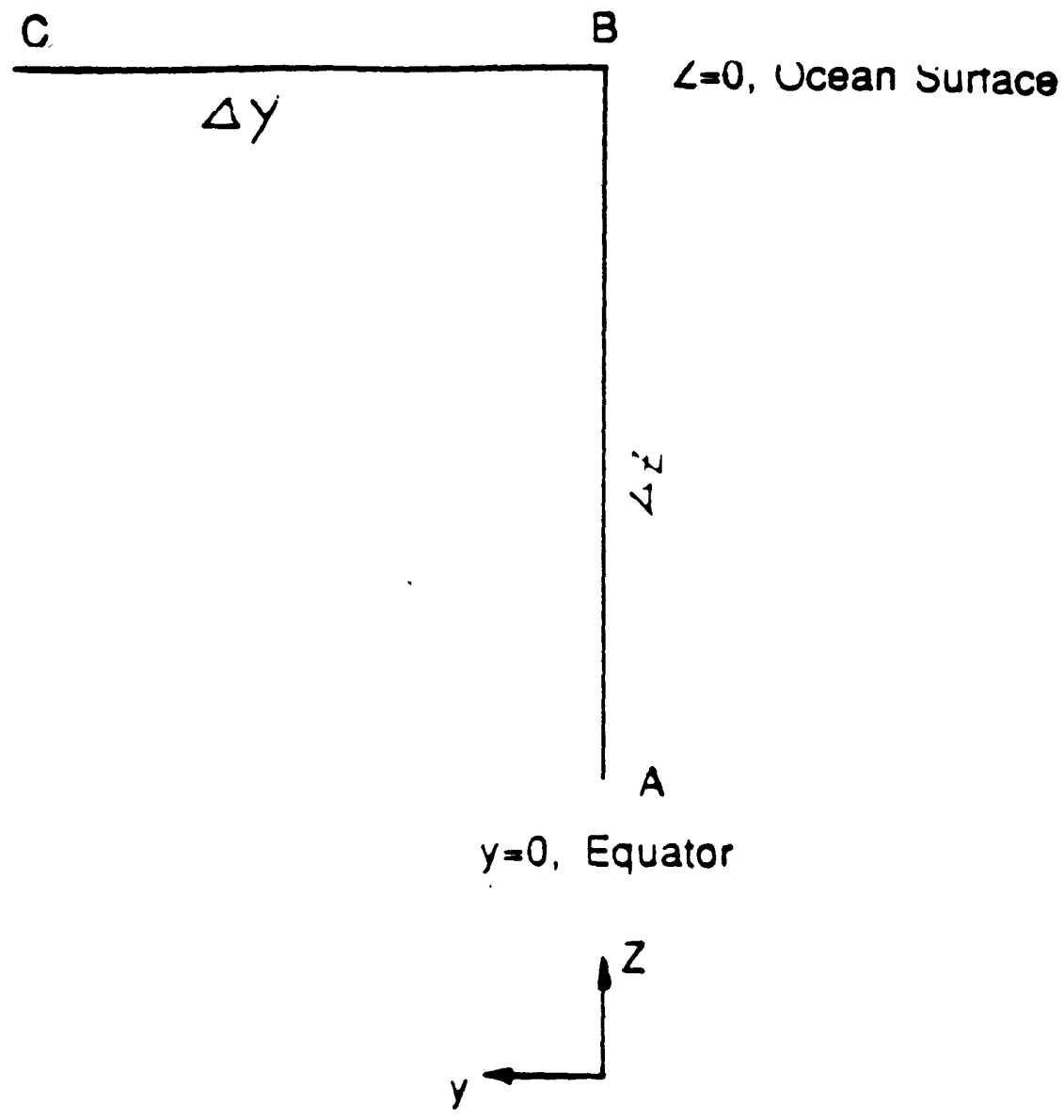

Fig.4.1. A schematic streamline in meridional plane A.B.C. The origin of the coordinate is at equator and ocean surface. 

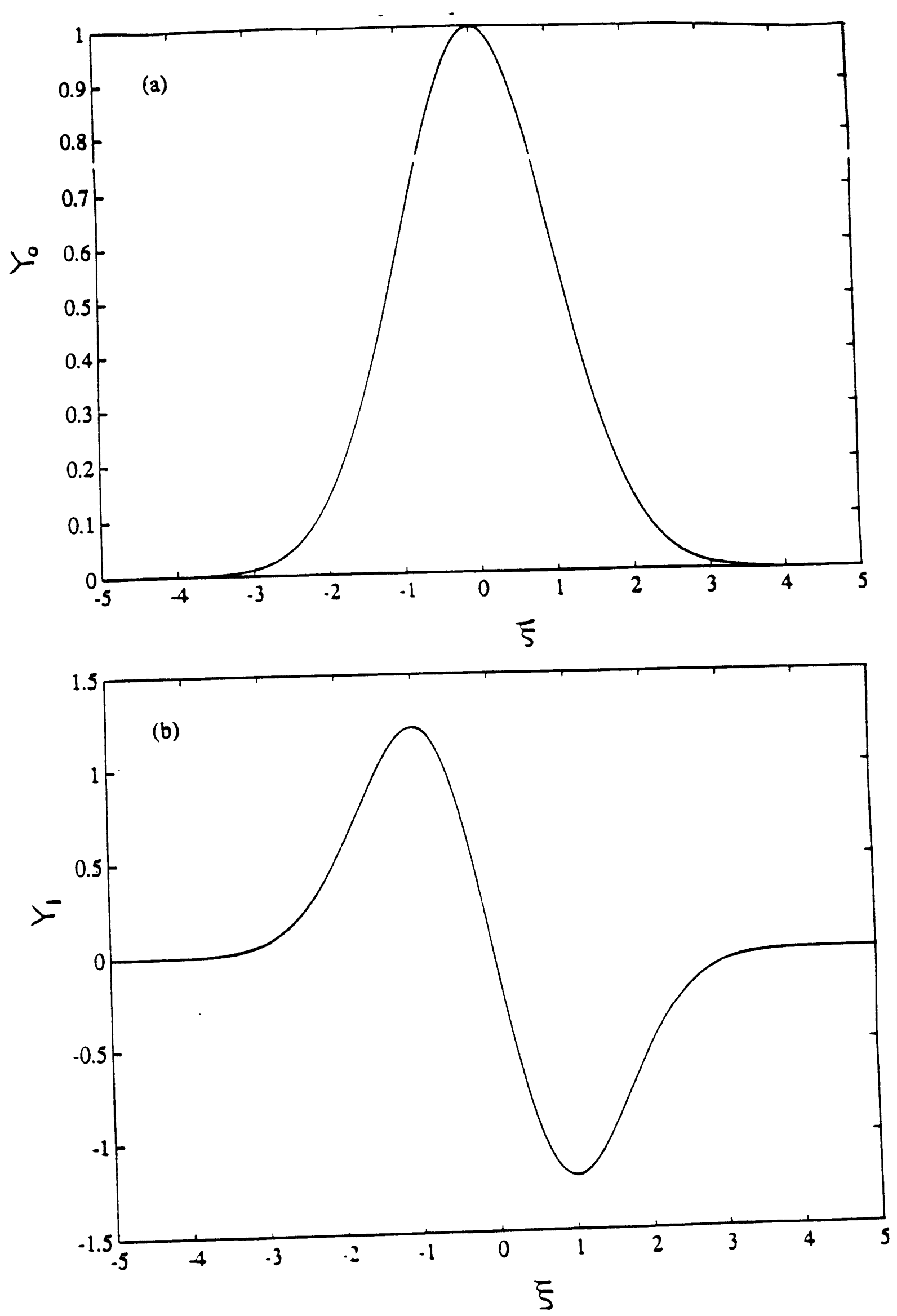


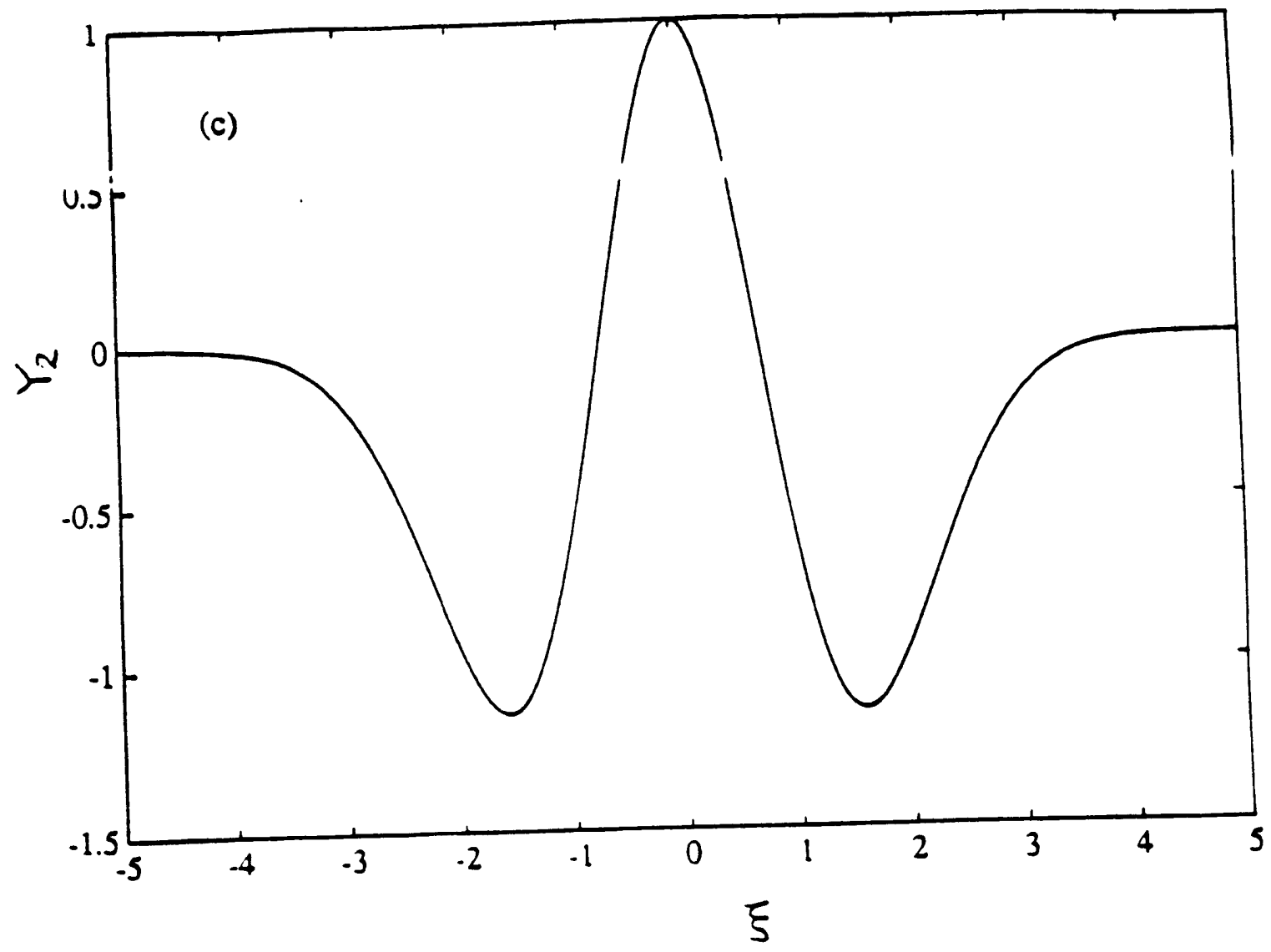

Fig.6.1. Horizontal structure eigenfunctions. (a) $j=0, Y_{0}=e^{-\frac{\xi^{2}}{2}}$. (b) $j=1, Y_{1}=2 \xi e^{-\frac{\xi^{2}}{2}}$.

(c) $j=2, Y_{2}=\left(2 \xi^{2}-1\right) \times e^{-\frac{\xi^{2}}{2}}$. 


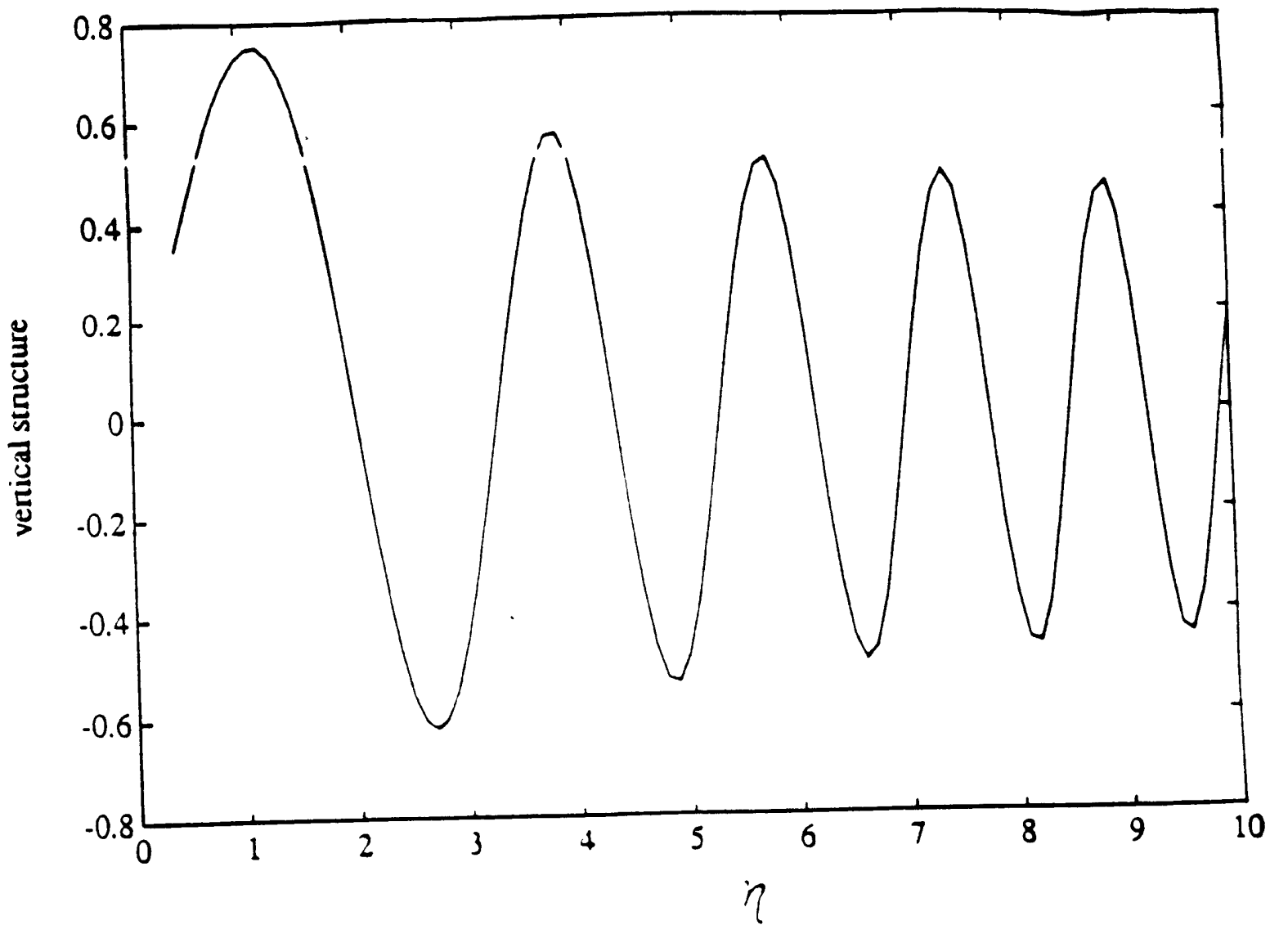

68

Fig.6.2. Vertical structure eigenfunctions. $Z(\eta)=\eta^{1 / 2} J_{1 / 3}\left(\frac{2}{3} \eta^{3 / 2}\right)$ 

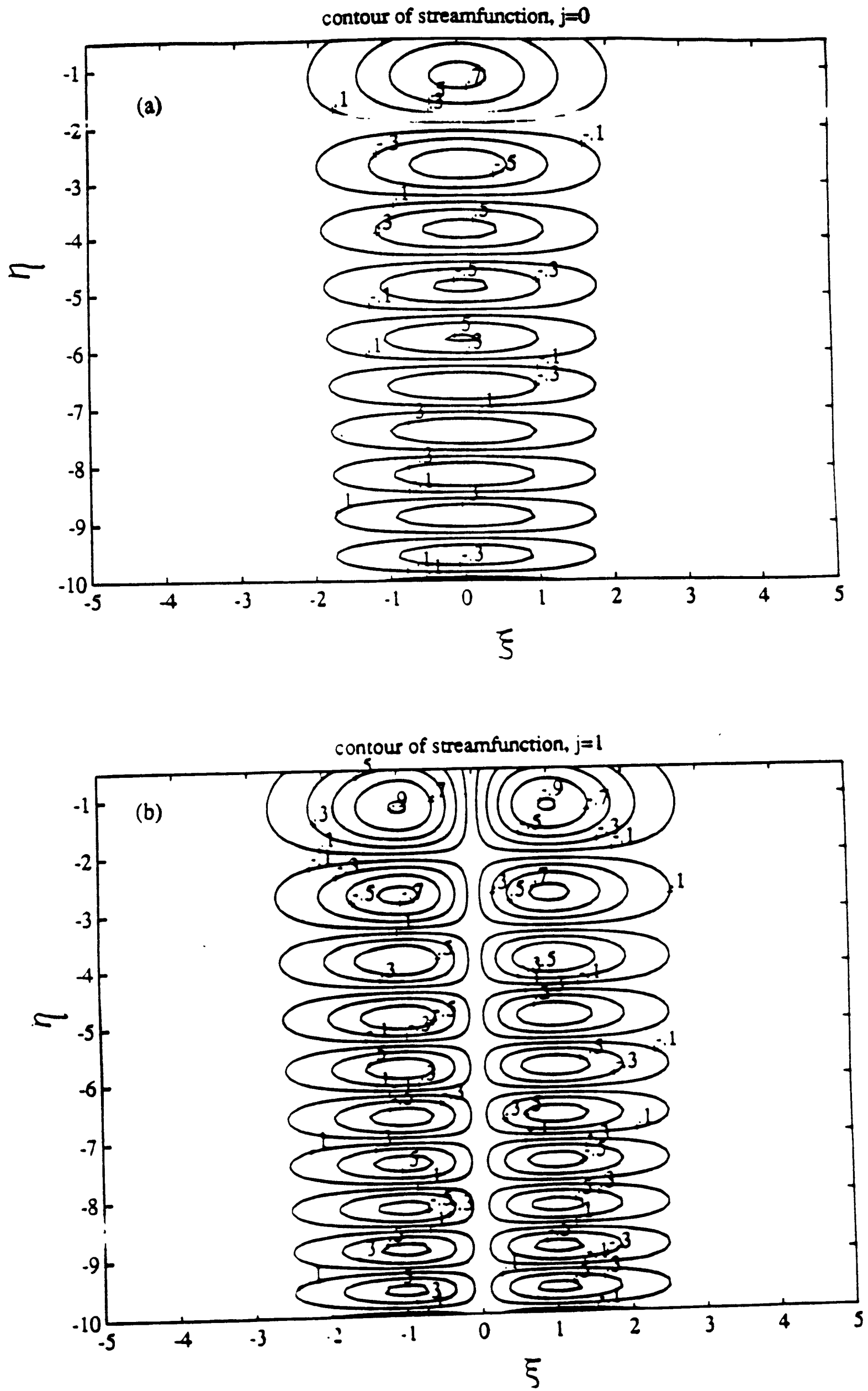


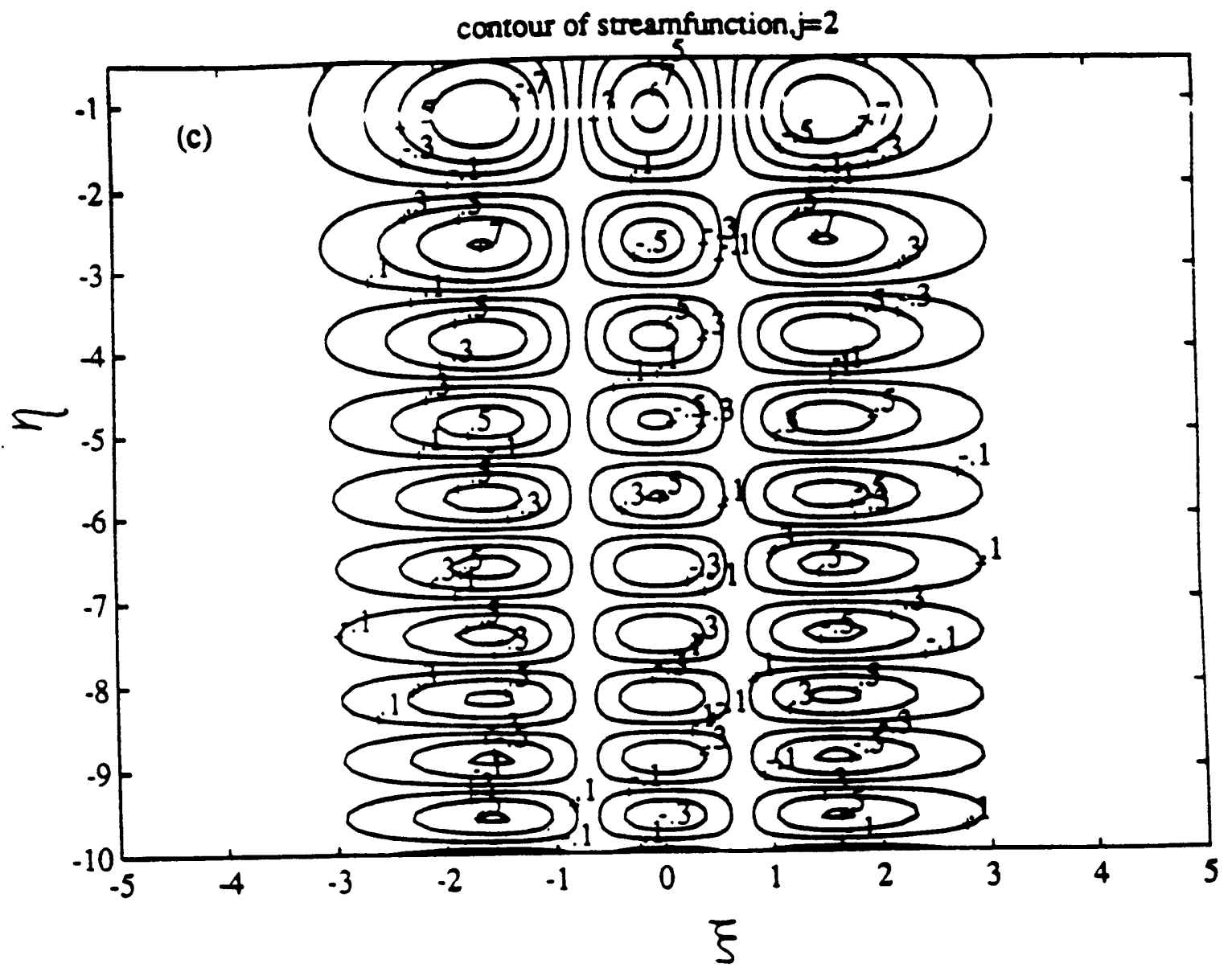

Fig.6.3. Contour of meridional streamfunctions (a) $j=0,(b) j=1$, (c) $j=2$. 


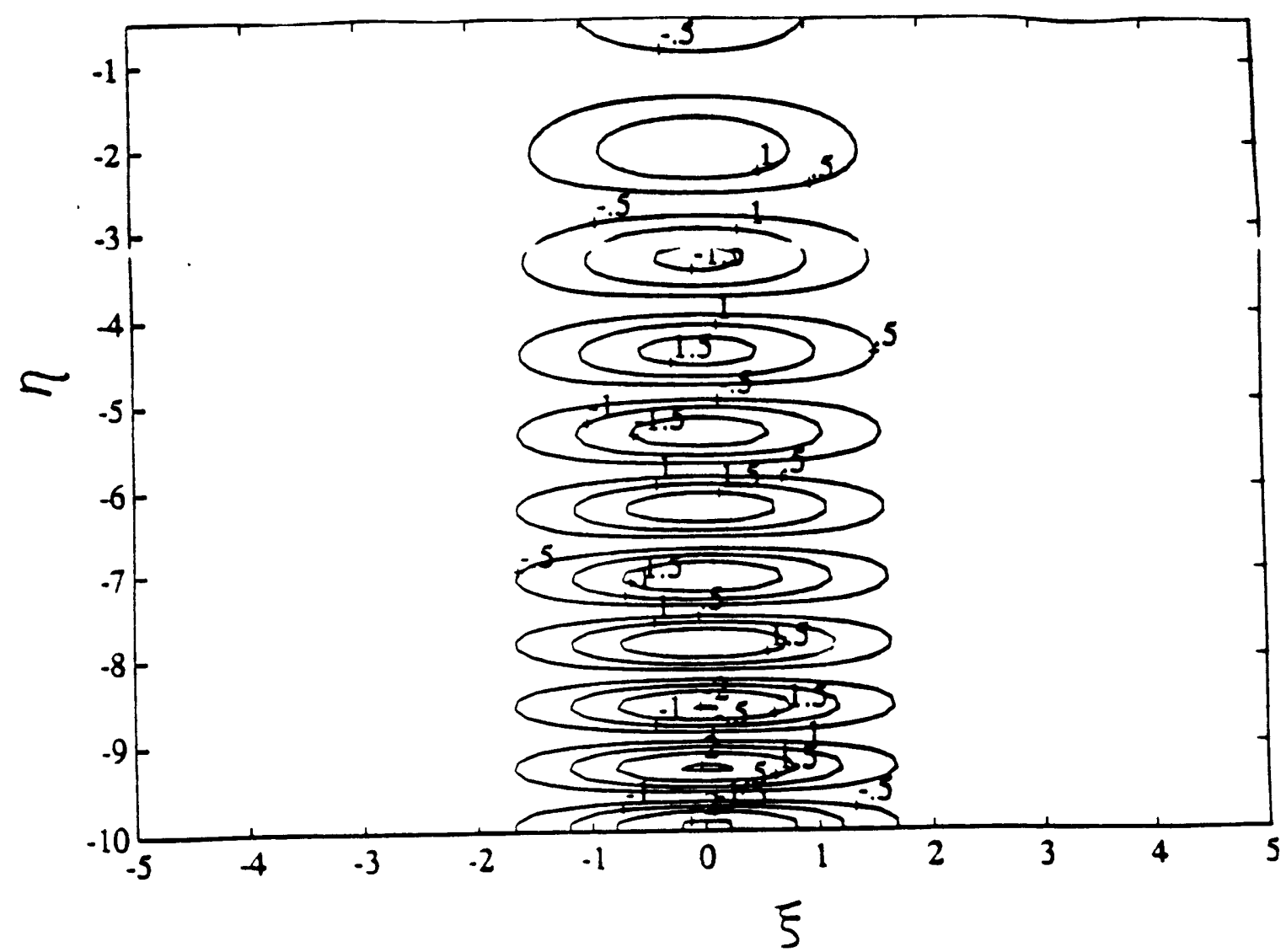

Fig.6.4. Contour of meridional velocity mode $\mathrm{j}=0$ in meridional plane.

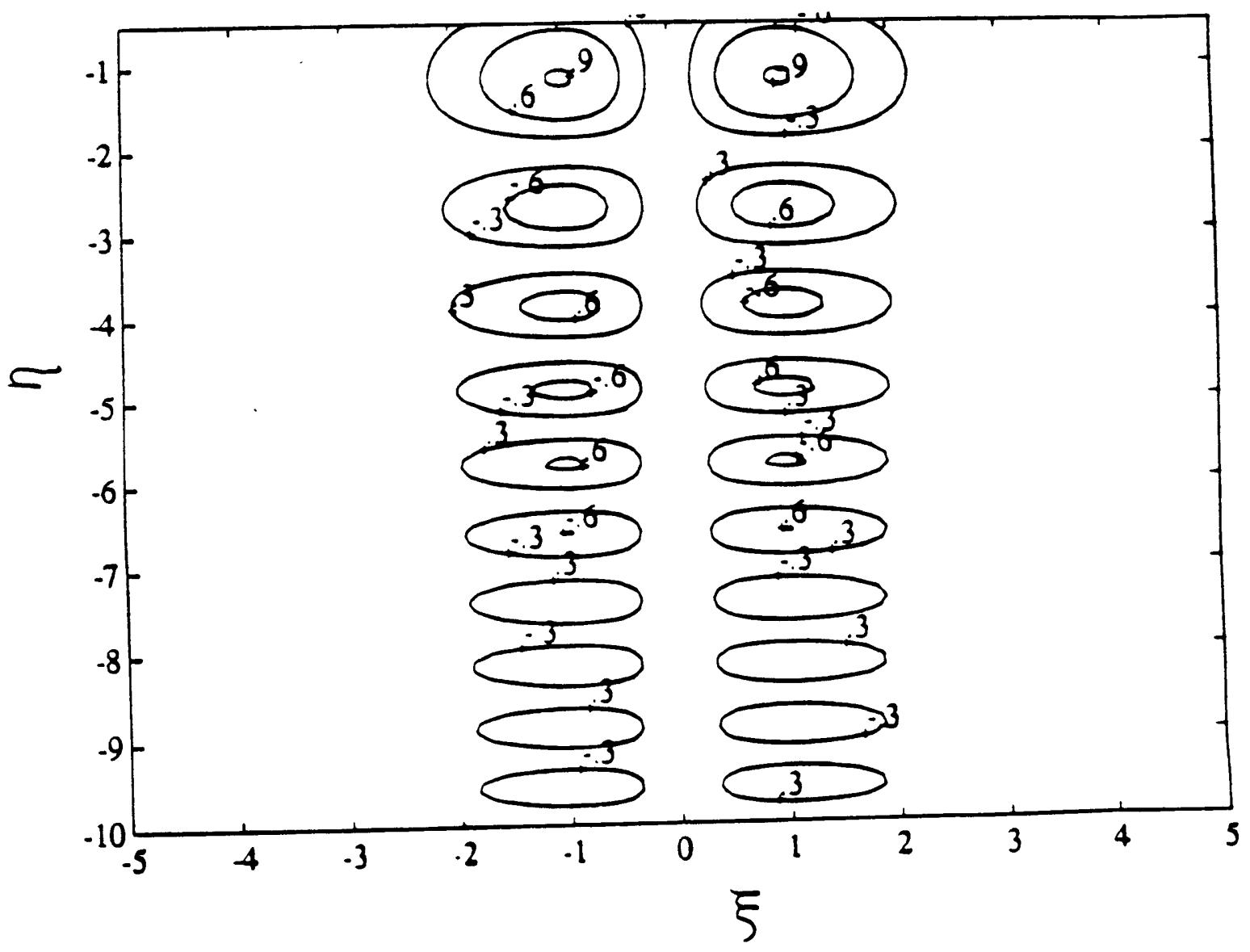

Fig.6.5. Contour of vertical velocity mode $j=0$ in meridional plane. 


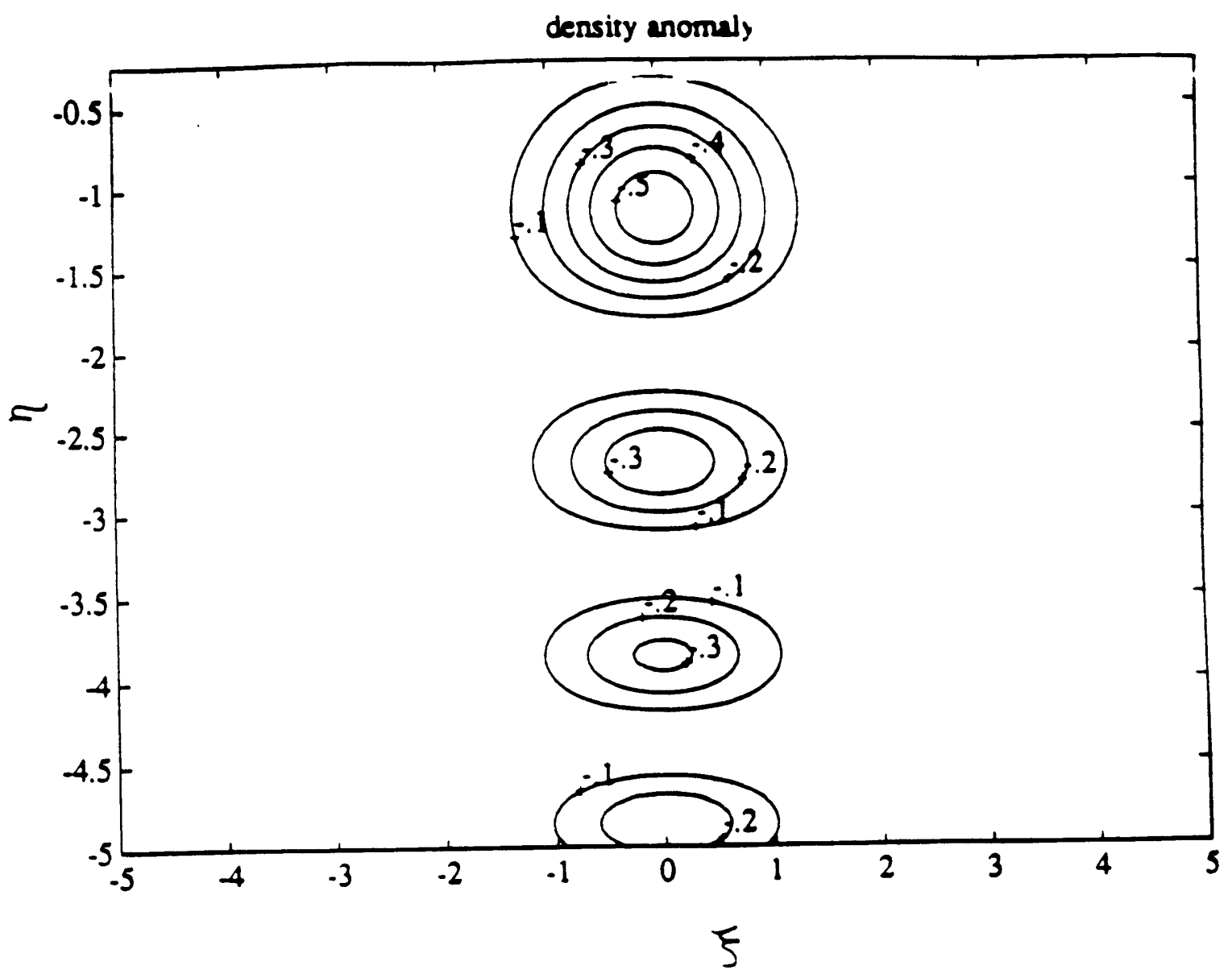

Fig.6.6. Meridional section of density anomaly $\phi^{2} / 2$. 


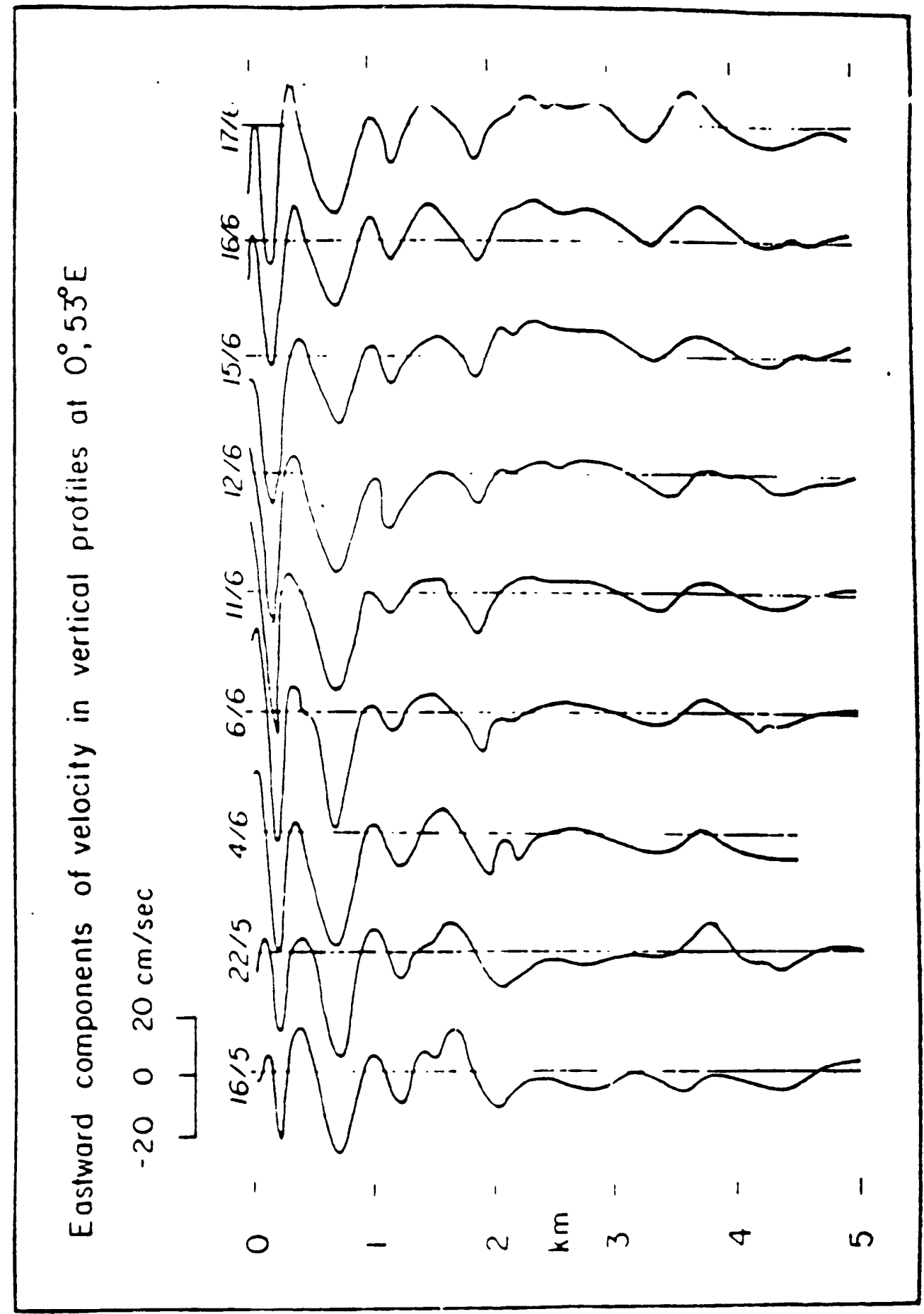

Fig.6.7. Vertical profiles of zonal velocity at Indian Ocean. (from Luyten and Swallow, 1976) 

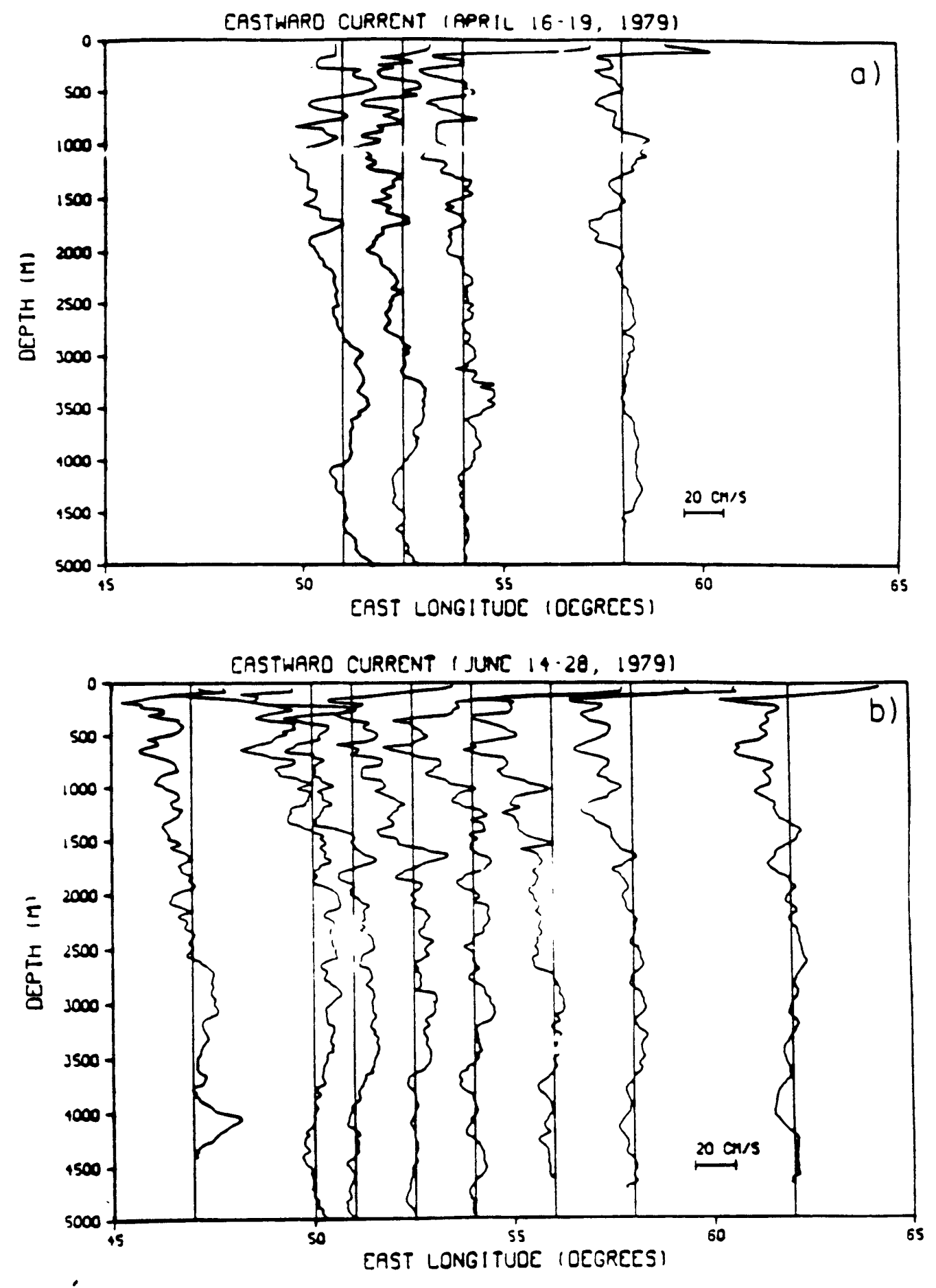

Fig.6.8. Zonal velocity along the equator measured during (a) April, 1979, (b) June, 1979. (from Ponte and Luyten, 1990) 


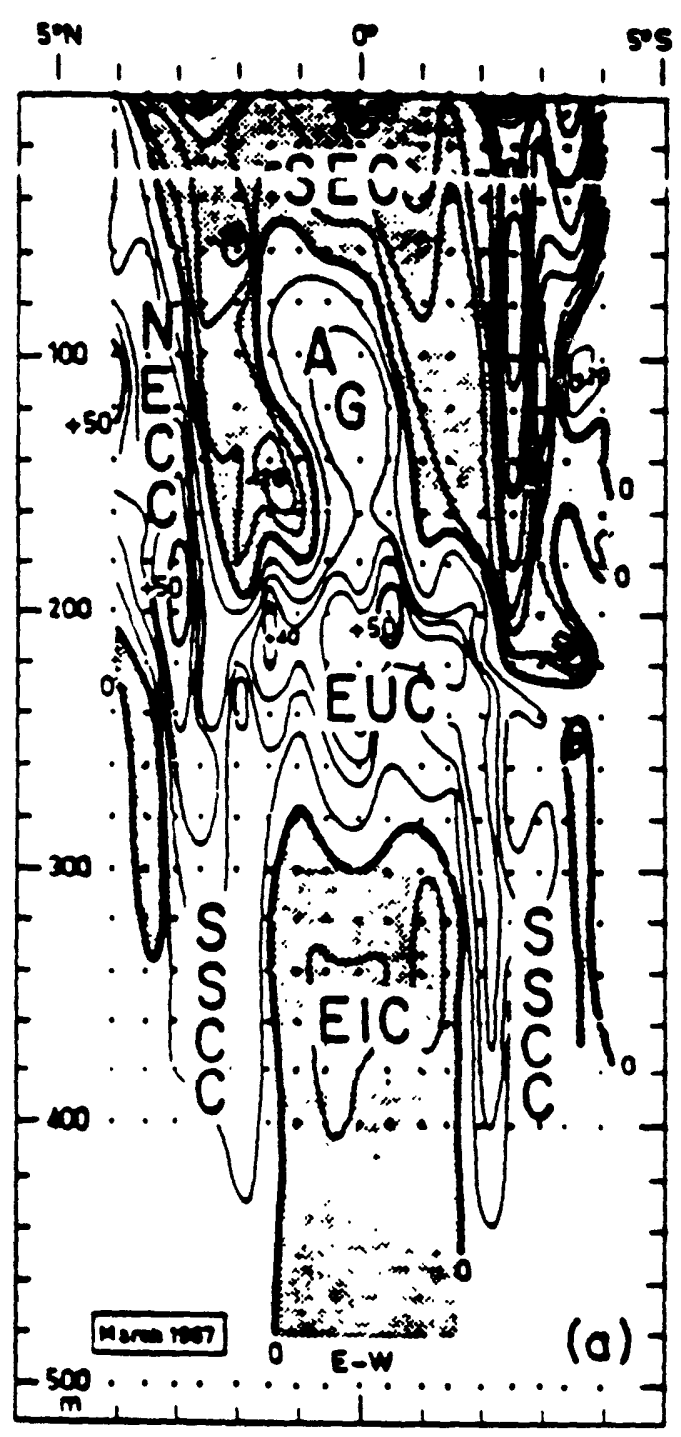

Fig.6.9. Meridional section of zonal velocity at $170^{\circ}$ E. Labeled currents are South Equatorial Current (SEC), Equatorial Undercurrent (EUC), Equatorial Intermediate Current (EIC), North Equatorial Countercurrent (NECC) and subsurface countercurrent (SSCC). The current labelled AG is an agostrophic flow. (from Hisard et al., 1970) 


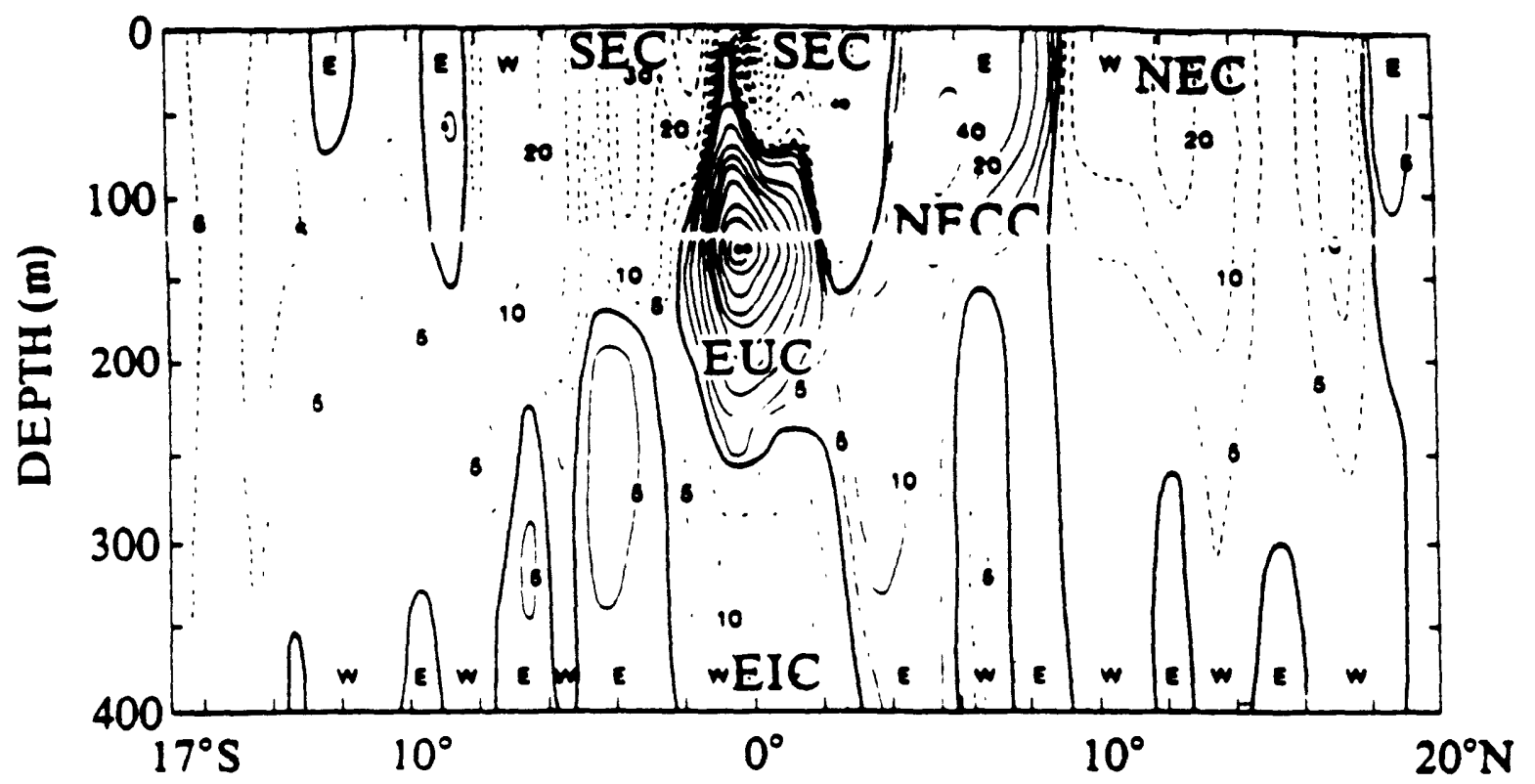

Fig.6.10. Contours of geostrophic zonal velocity $(\mathrm{cm} / \mathrm{s})$. Shown is the mean average actoss $150^{\circ} \mathrm{W}-158^{\circ} \mathrm{W}$ based on 15 cruises. Solid: eastward, dashed: westward. (from Wyrki and Kilonsky, 1984)

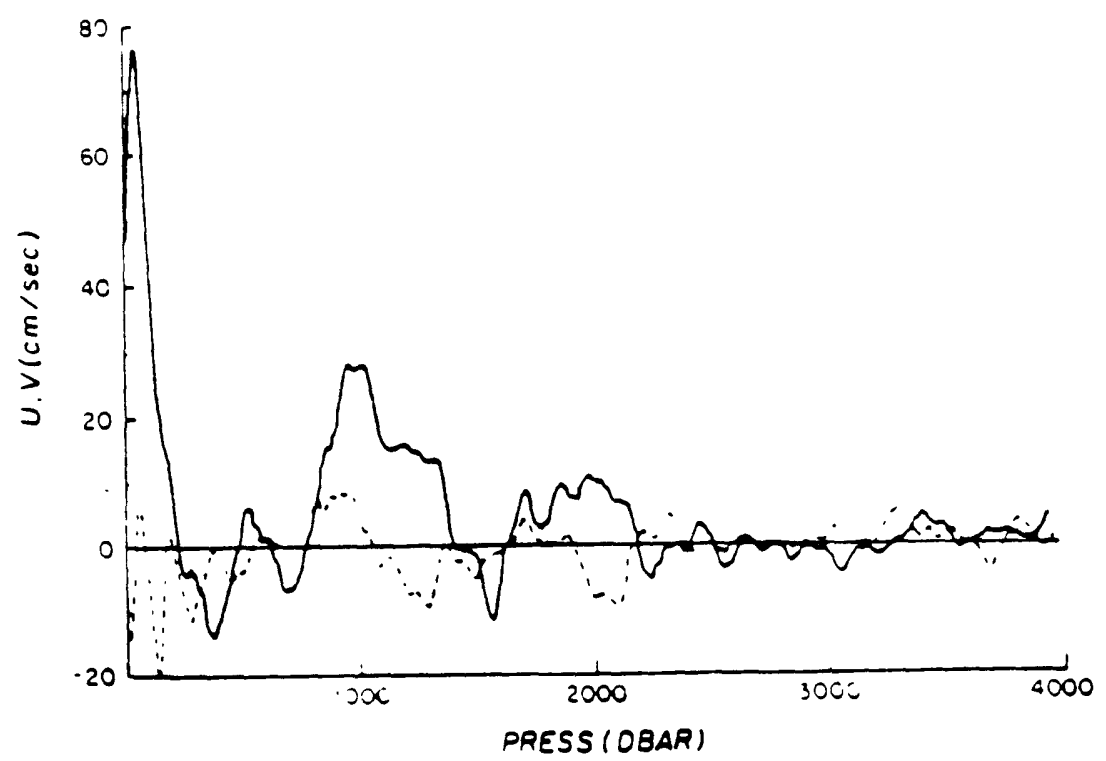

Fig.6.11. East and norh velocities $(\mathrm{cm} / \mathrm{s})$ as function of pressure (decibars), observed on 17 January, 1989 in the equatonal Atlantic at $0^{\circ} \mathrm{N}, 30^{\circ} 22 \mathrm{~W}$. (from Ponte, Luyten and Richardson, 1990). 

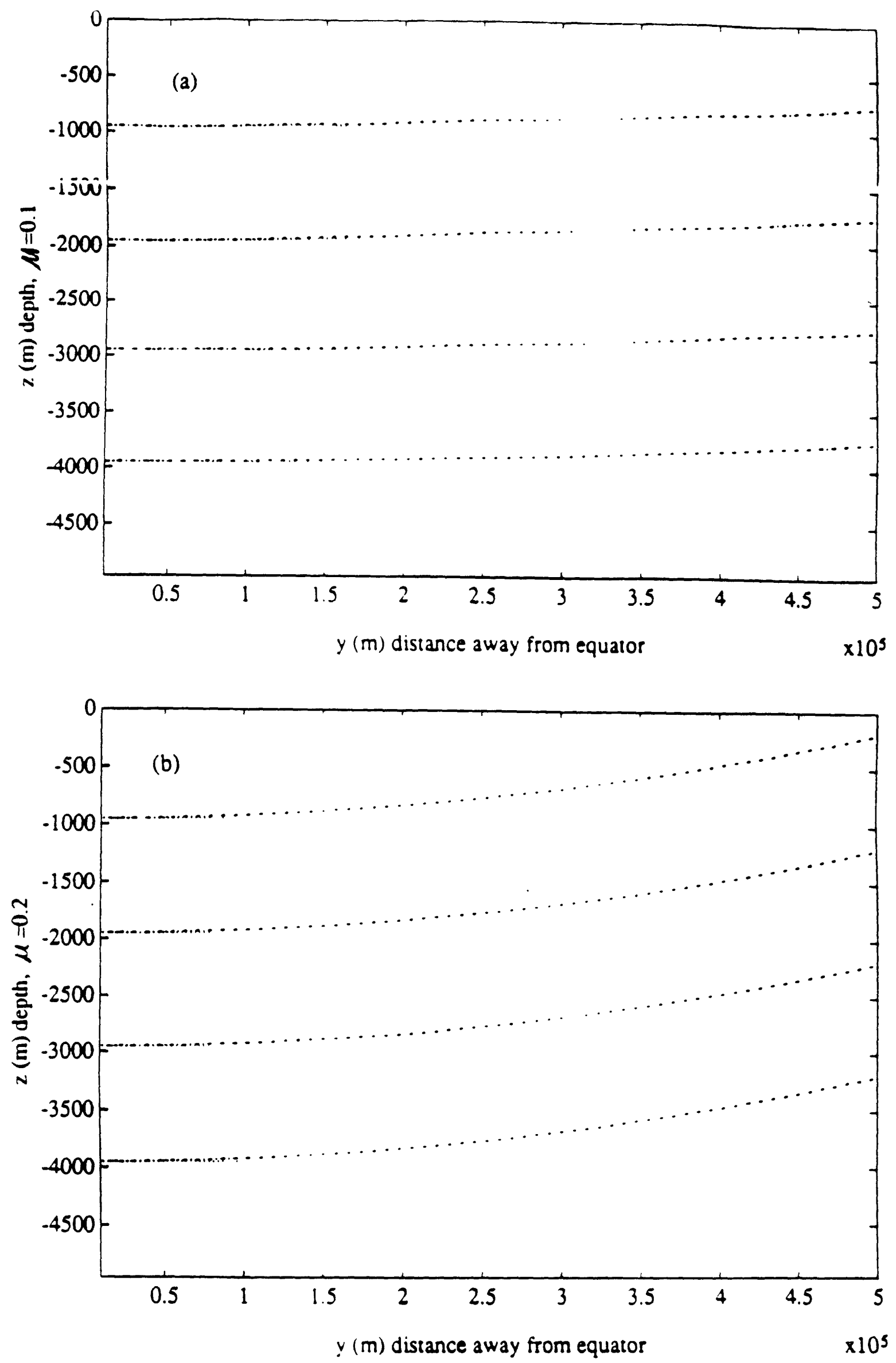

Fig.7.1. Comparison of modufied depth (dorted line) and real depth (dashdor line). (a) $\mu=0.1$, (b) $\mu=0.2$. 


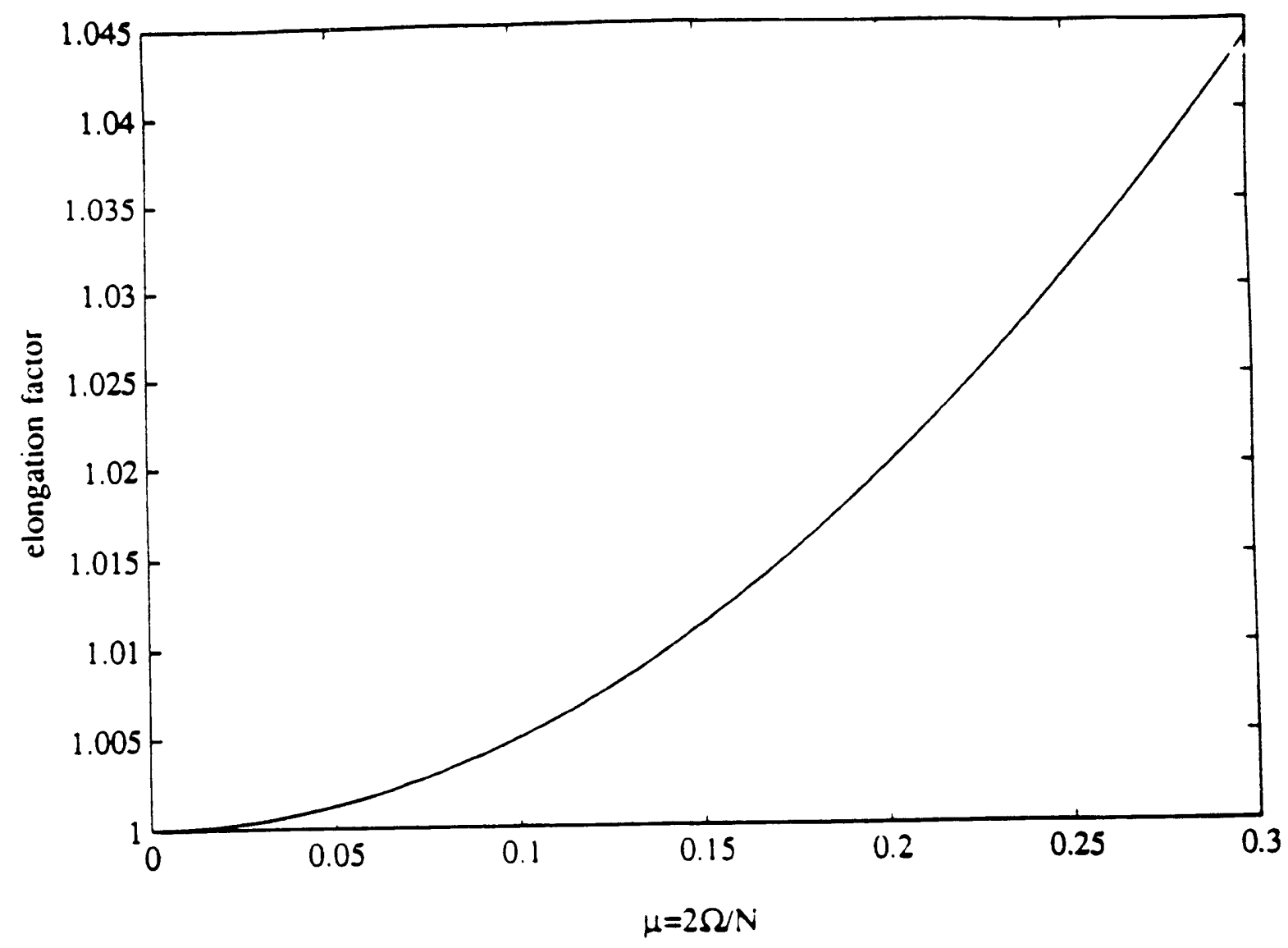

Fig.7.2. Elongation factor as a function of $\mu$. 

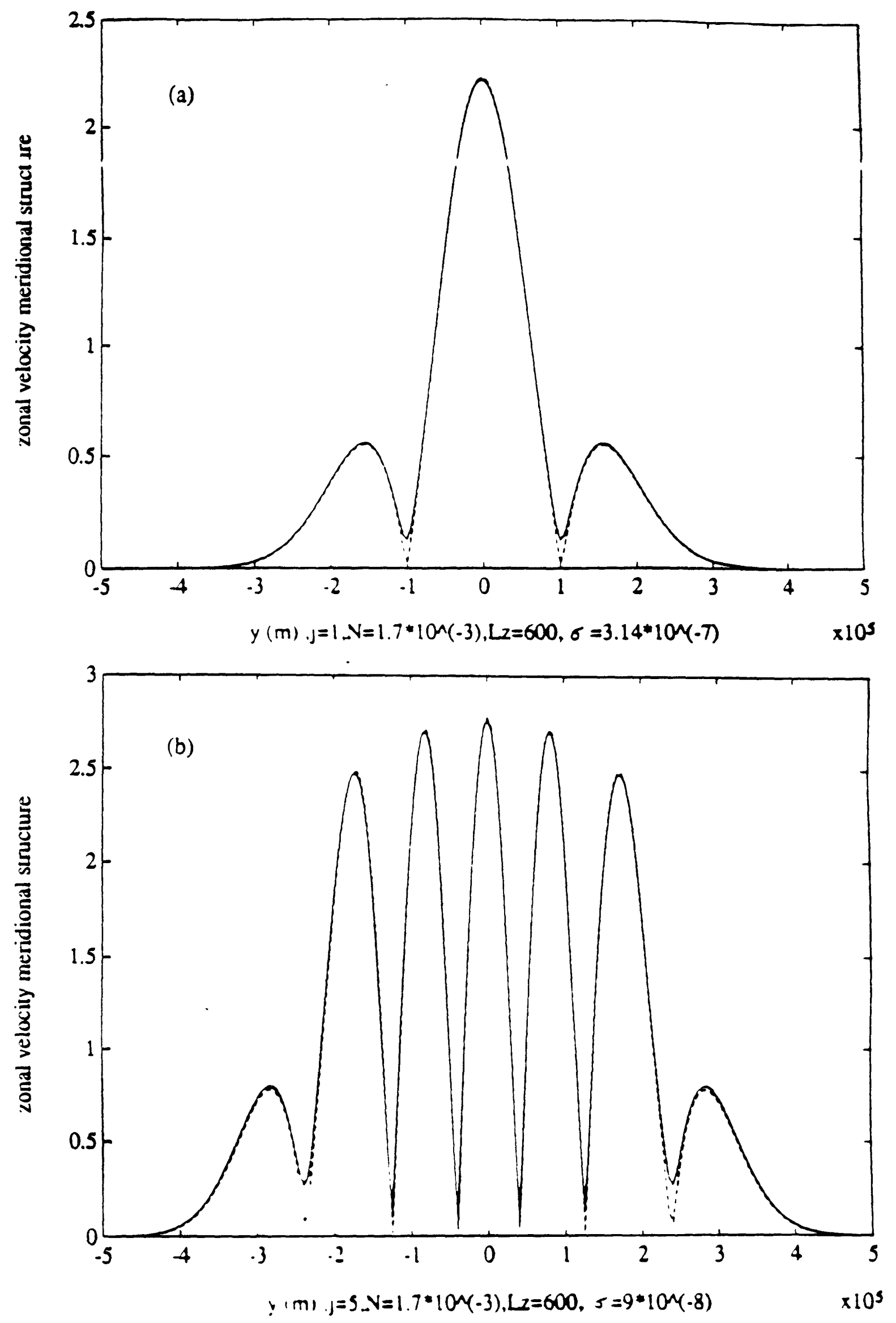

Fig.7.3. Comparison of modified ( slolid) and unmodified (dash) zonal velocity meridional structure only when moridional mocke number changes. (a) for $j=1$, (b) for $j=5$. All other parameters are the same as in viandard see 

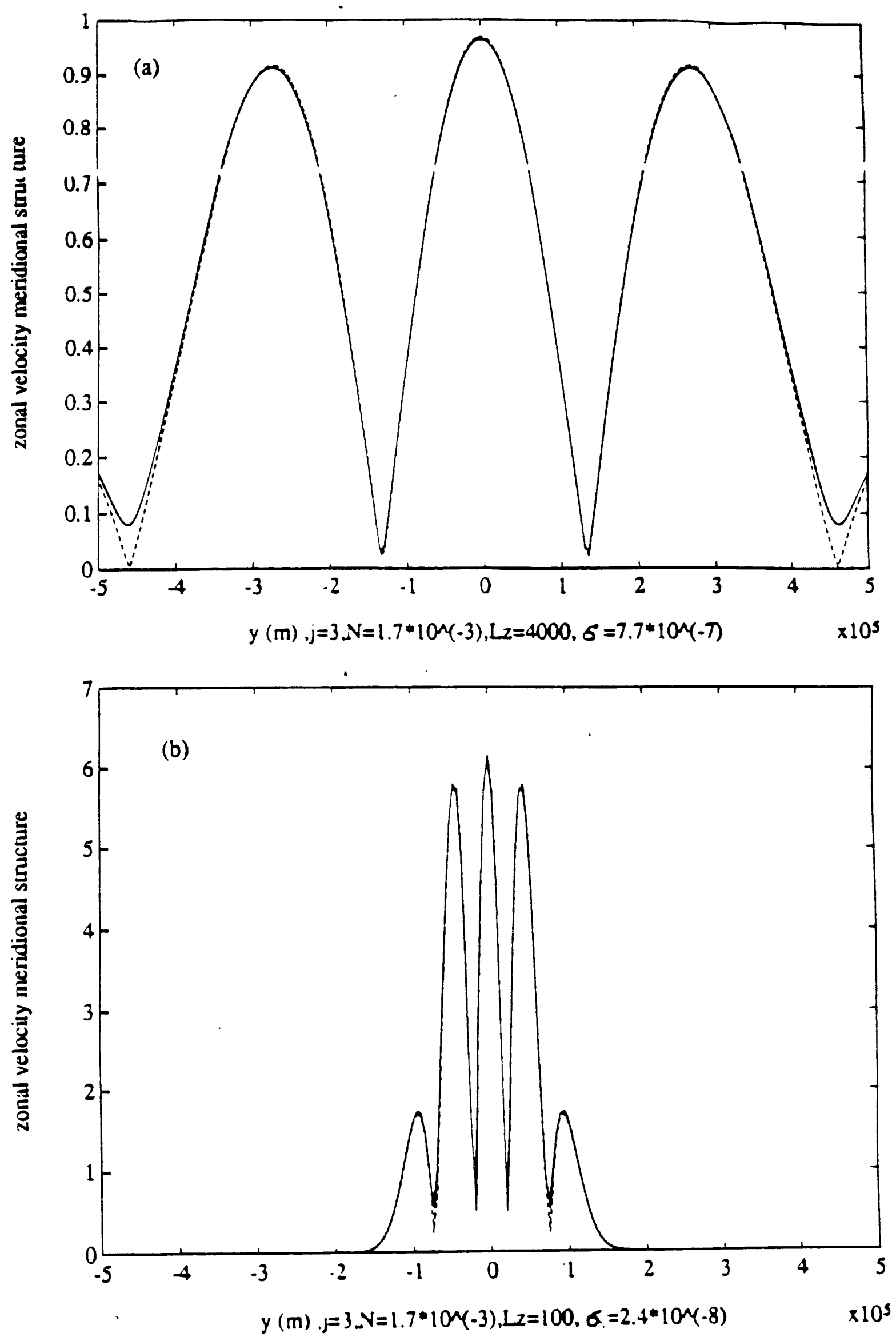

Fig.7.4. Same as in Fig. 7.3 excepe only verical wavelength changes. (a) $L_{2}=4000 \mathrm{~m}$, (b) $\mathrm{L}_{\mathrm{z}}=100 \mathrm{~m}$. 

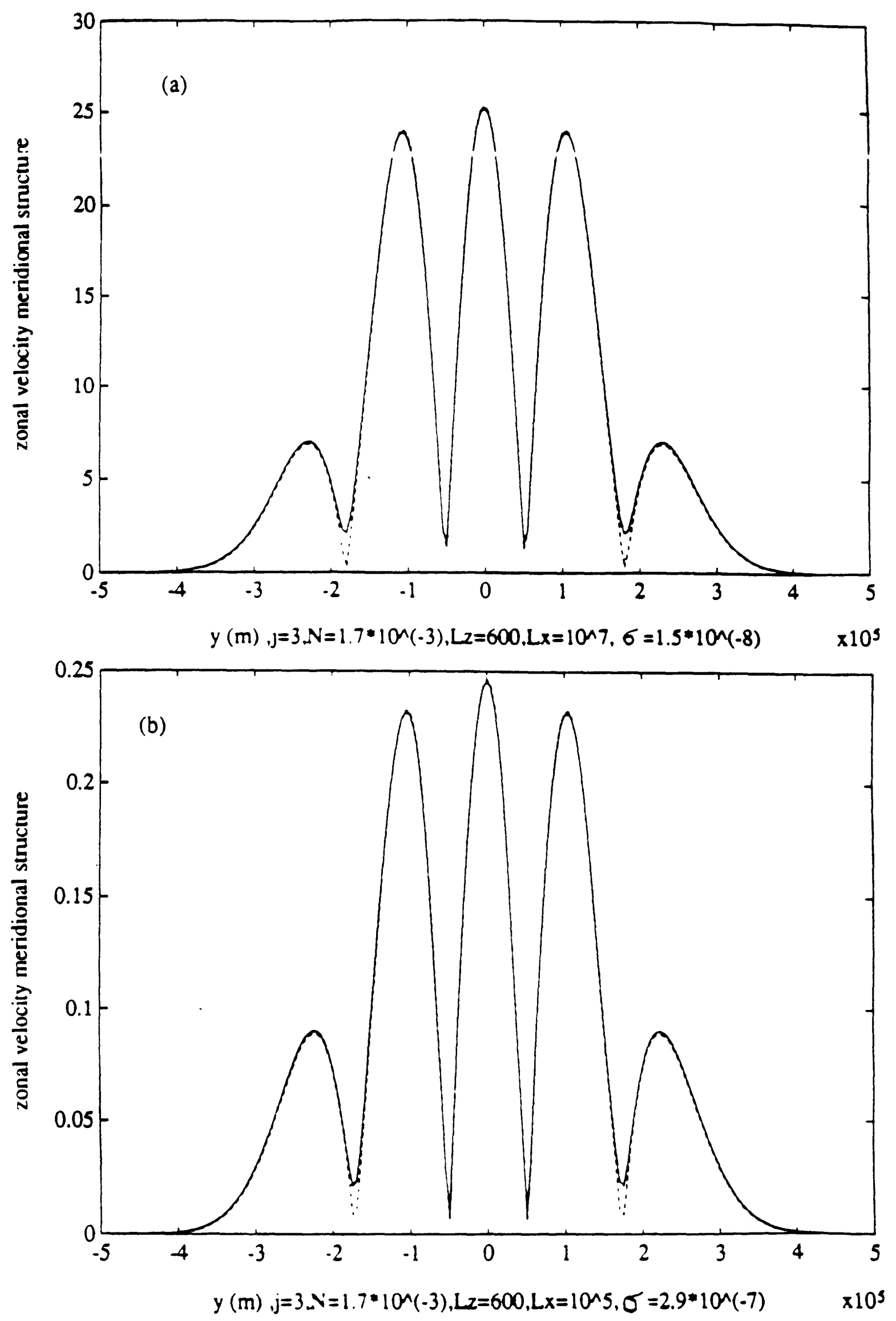

Fig.7.5. Same as in Fig.7.3 except only zonal wavelength changes. (a) $L_{x}=10^{7} \mathrm{~m}$, (b) $L_{x}=10^{5} \mathrm{~m}$. 


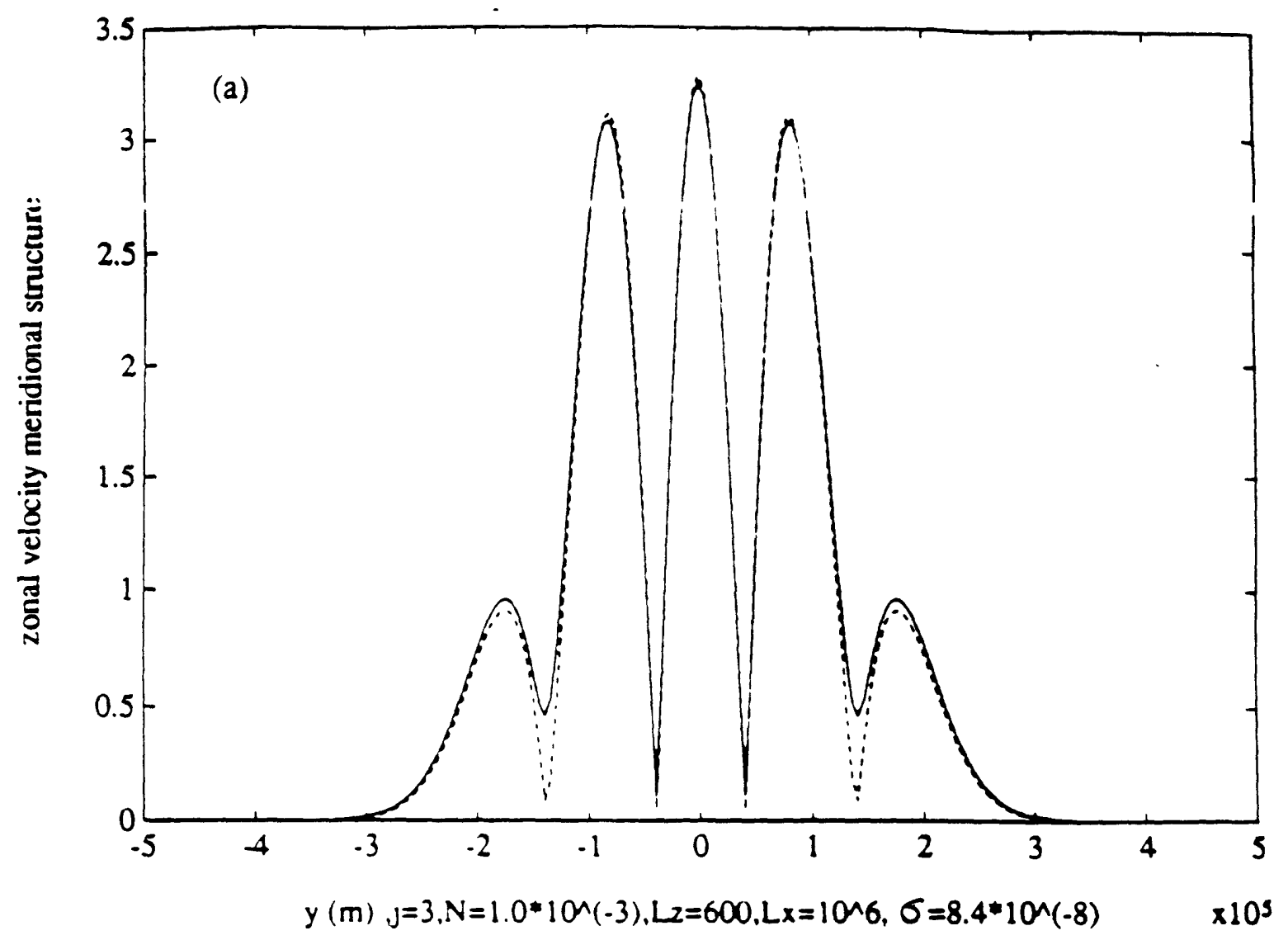

82

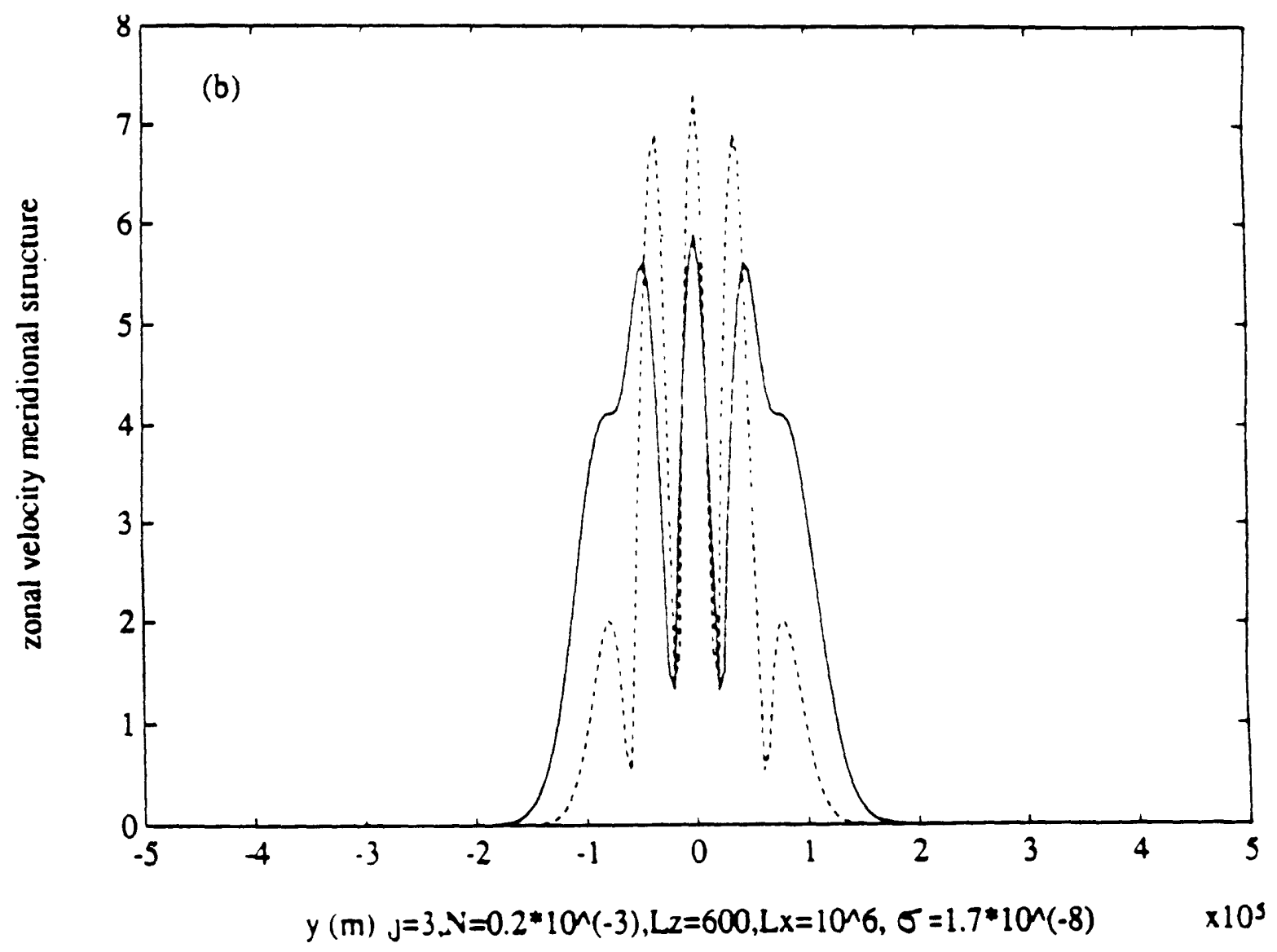

Fig.7.6. Same as in Fig.7.3 except only the vertical stratification changes. (a) $N=10-3$ $\mathrm{rad} / \mathrm{s}$, (b) $\mathrm{N}=0.2 \times 10^{-3} \mathrm{rad} / \mathrm{s}$. 


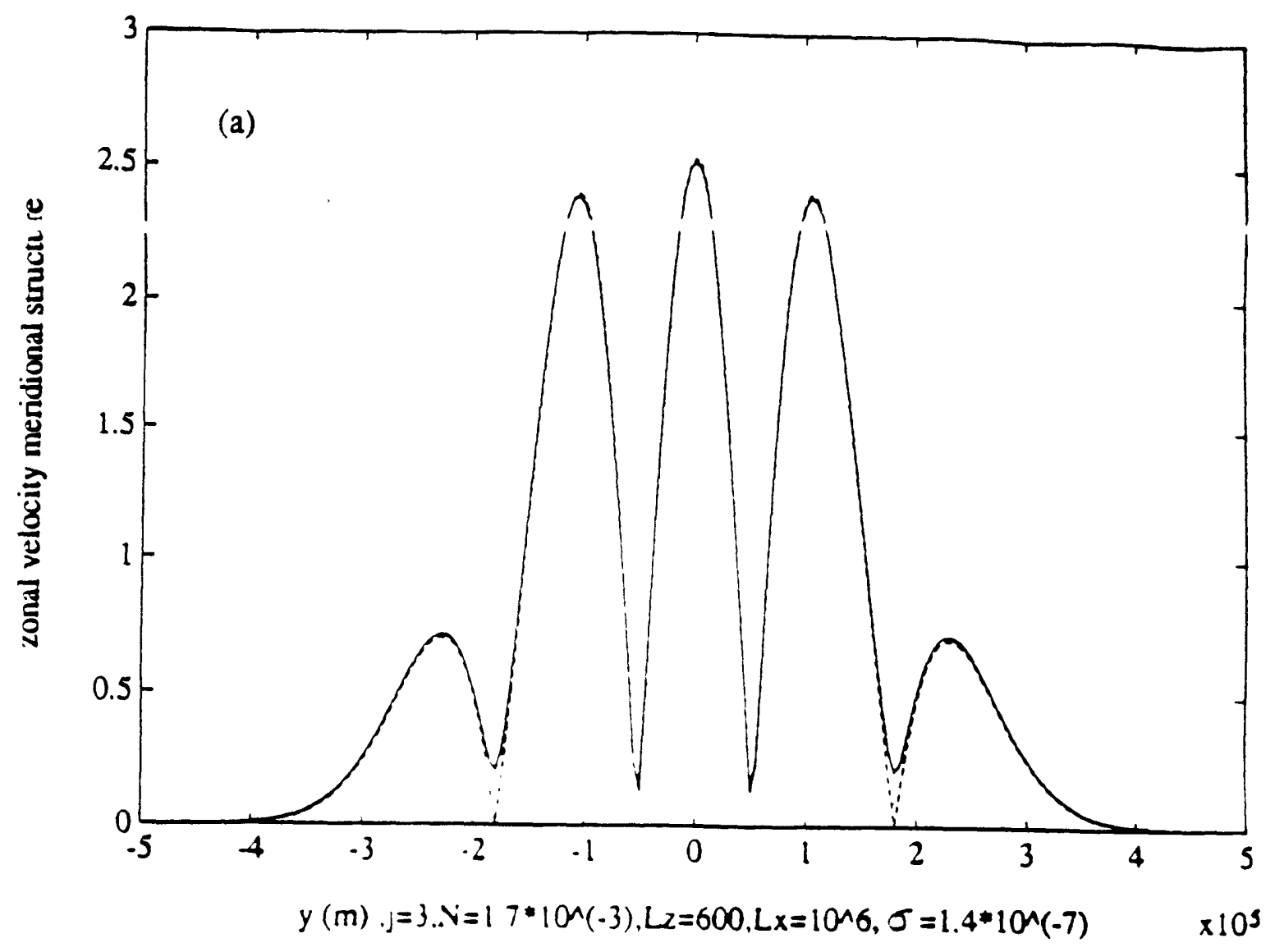

83

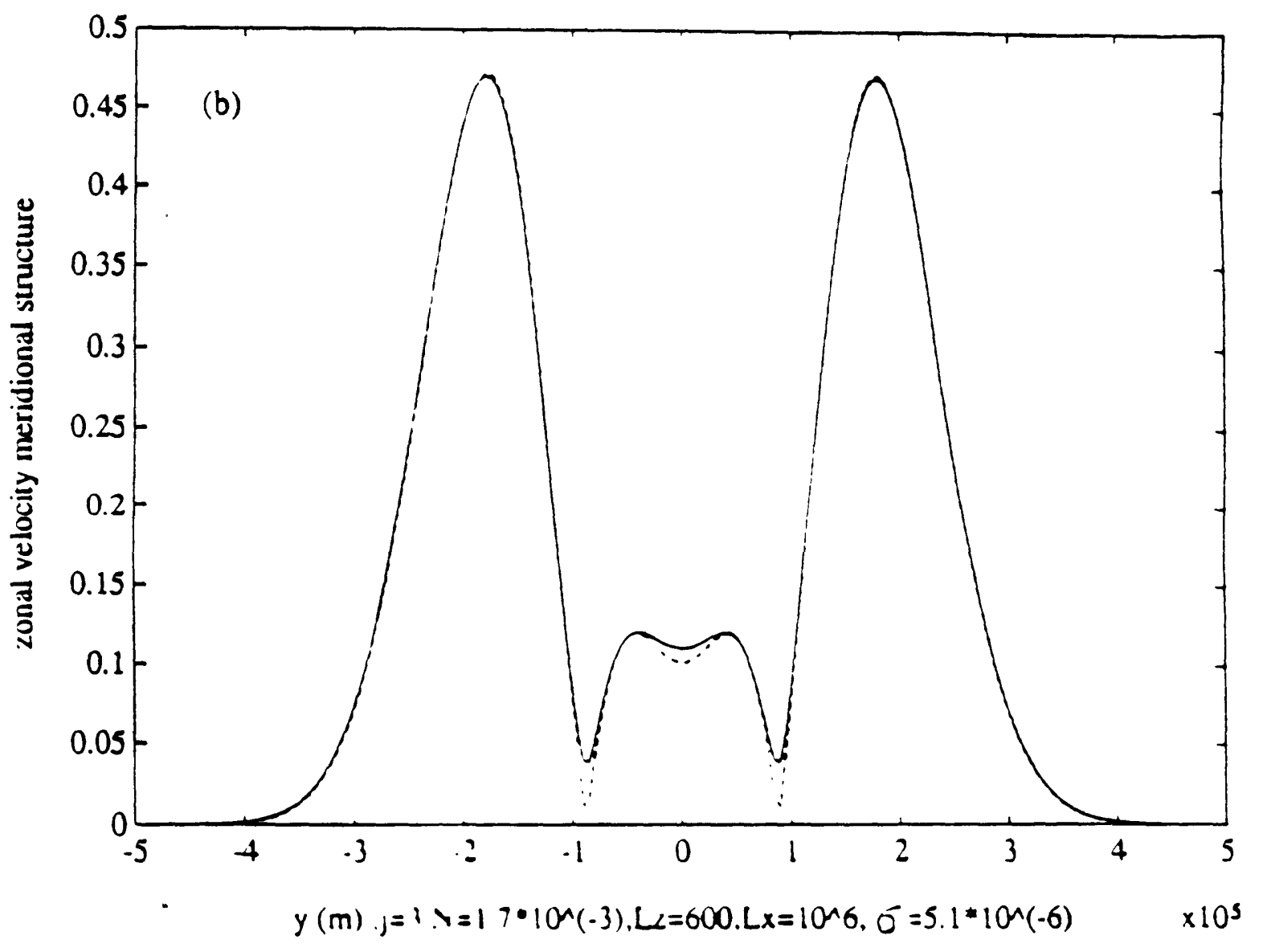

Fig.7.7. The comparison is mule for different types of waves under same standard parameter set. (a) $\sigma=1.4 \times 10^{\circ} \mathrm{r}$ s $/ \mathrm{s}$ ior lower frequency wave, (b) $\sigma=5.1 \times 10^{-6} \mathrm{rad} / \mathrm{s}$ for higher frequency wave. 


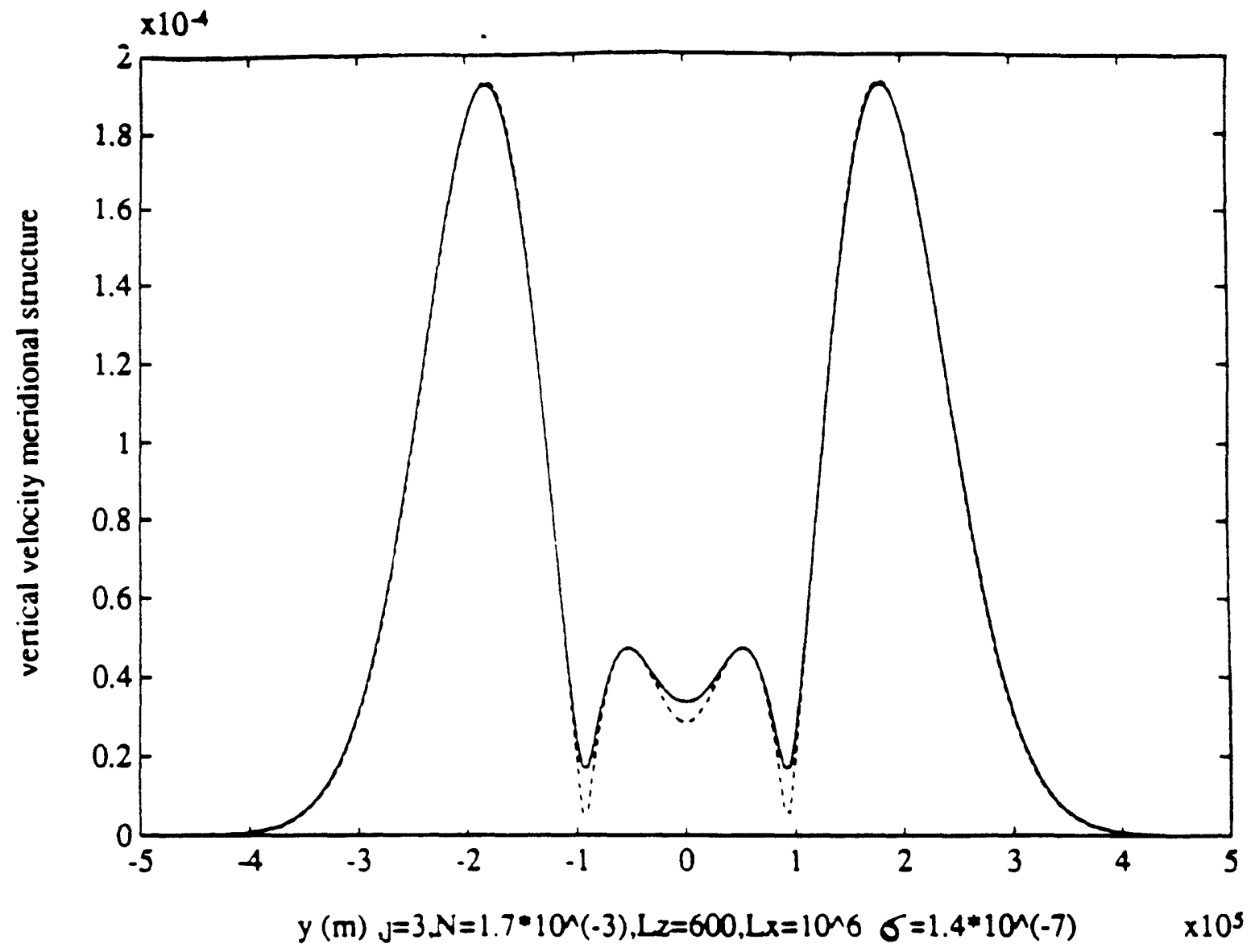

Fig.7.8. Comparison of modified (solid) and unmodified (dash) verrical velocity meridional structure under standard parameter set. 

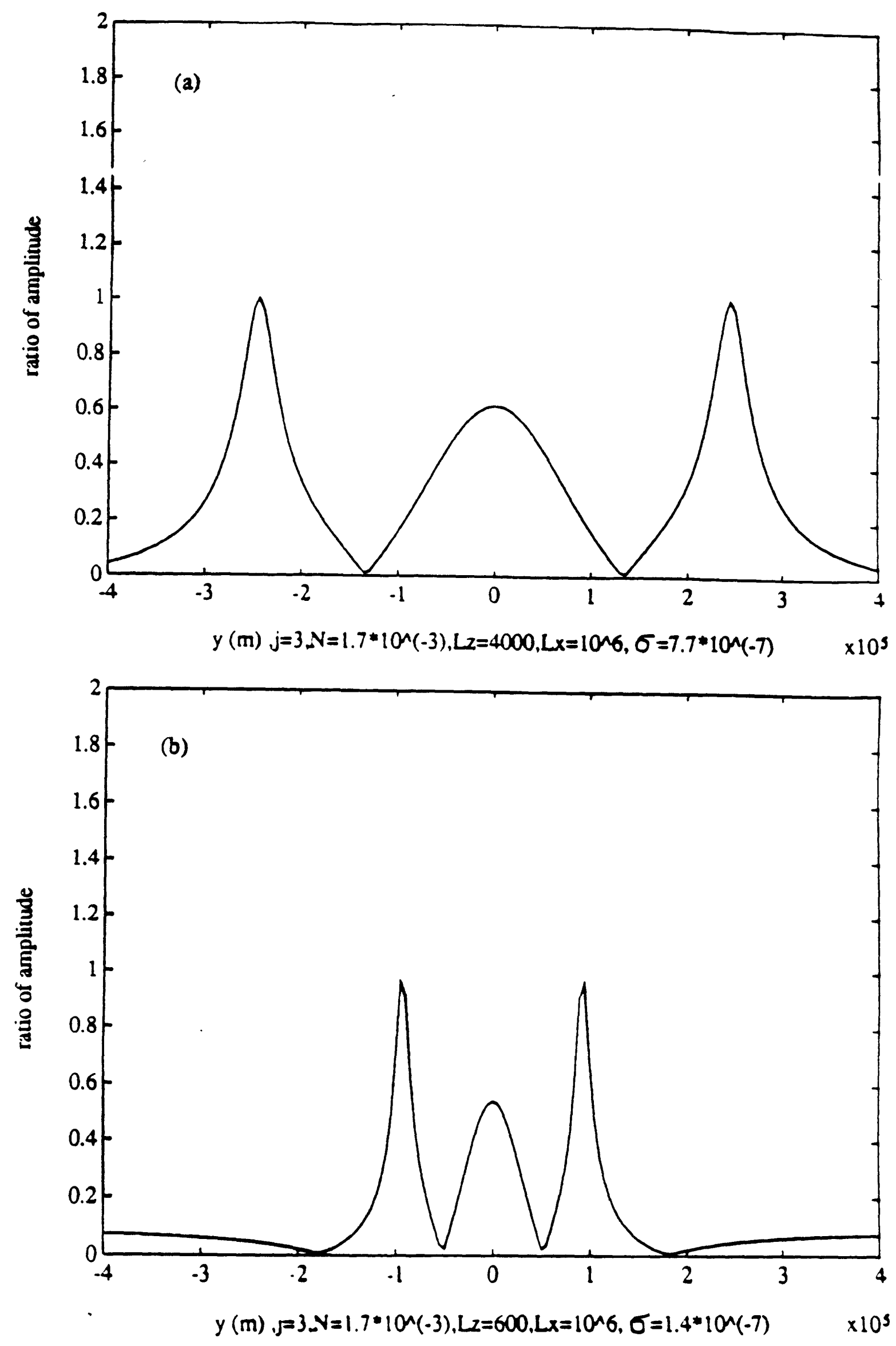

Fig.7.9. The meridional discribution of $\gamma$ for low frequency waves. The smallness of $\gamma$ characterizes the goodness of hydrostatic relacion. (a) $L_{z}=4000 \mathrm{~m}$, (b) $L_{z}=600 \mathrm{~m}$. 

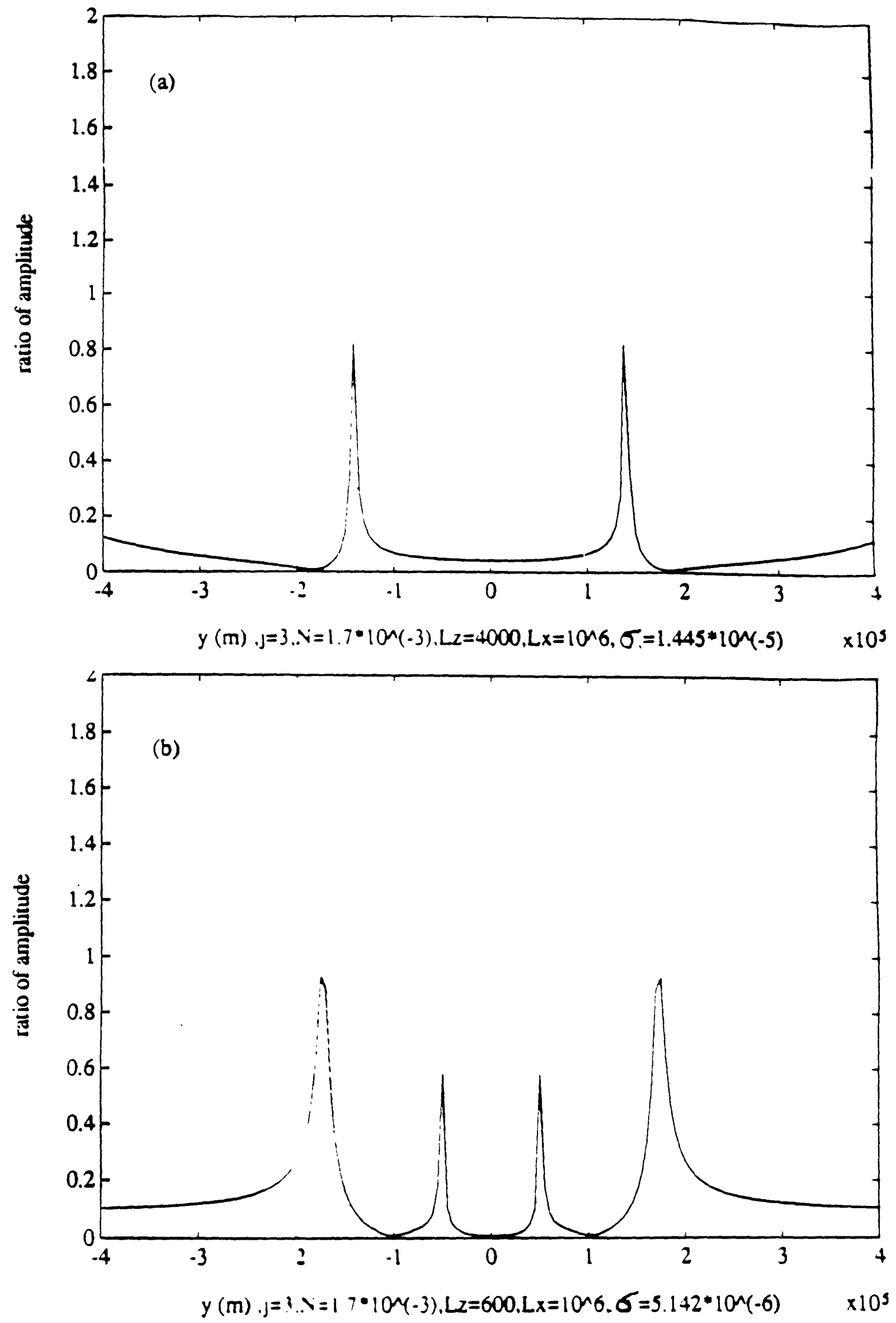

Fig.7.10. Same as Fig.7.9 er.ent lor higher frequency waves. 

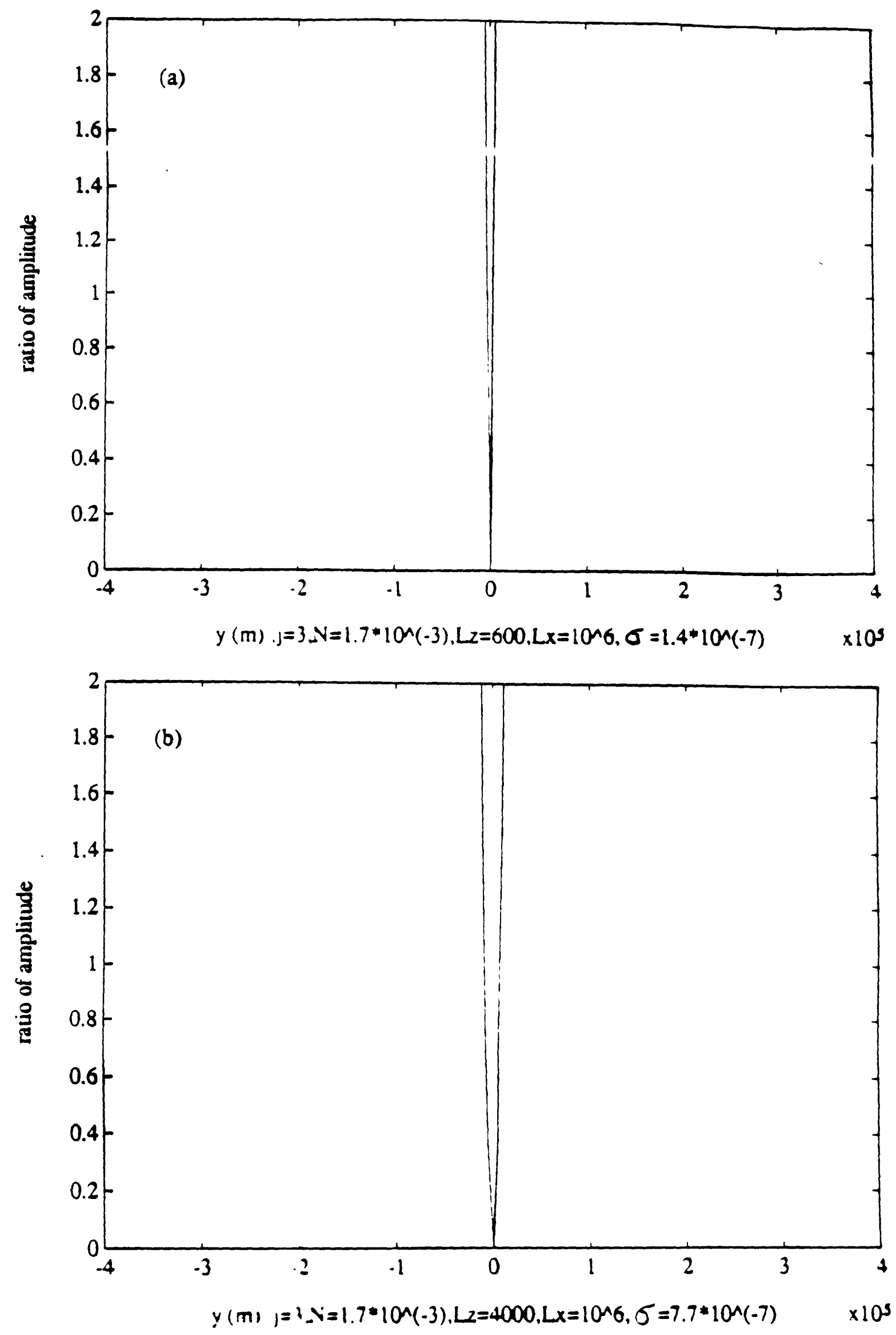

Fig. 7.11. The ratio $\frac{\beta y v}{f_{0} w}$ for lower frequency waves. (a) $L_{z}=600 \mathrm{~m}$, (b) $L_{z}=4000 \mathrm{~m}$. 

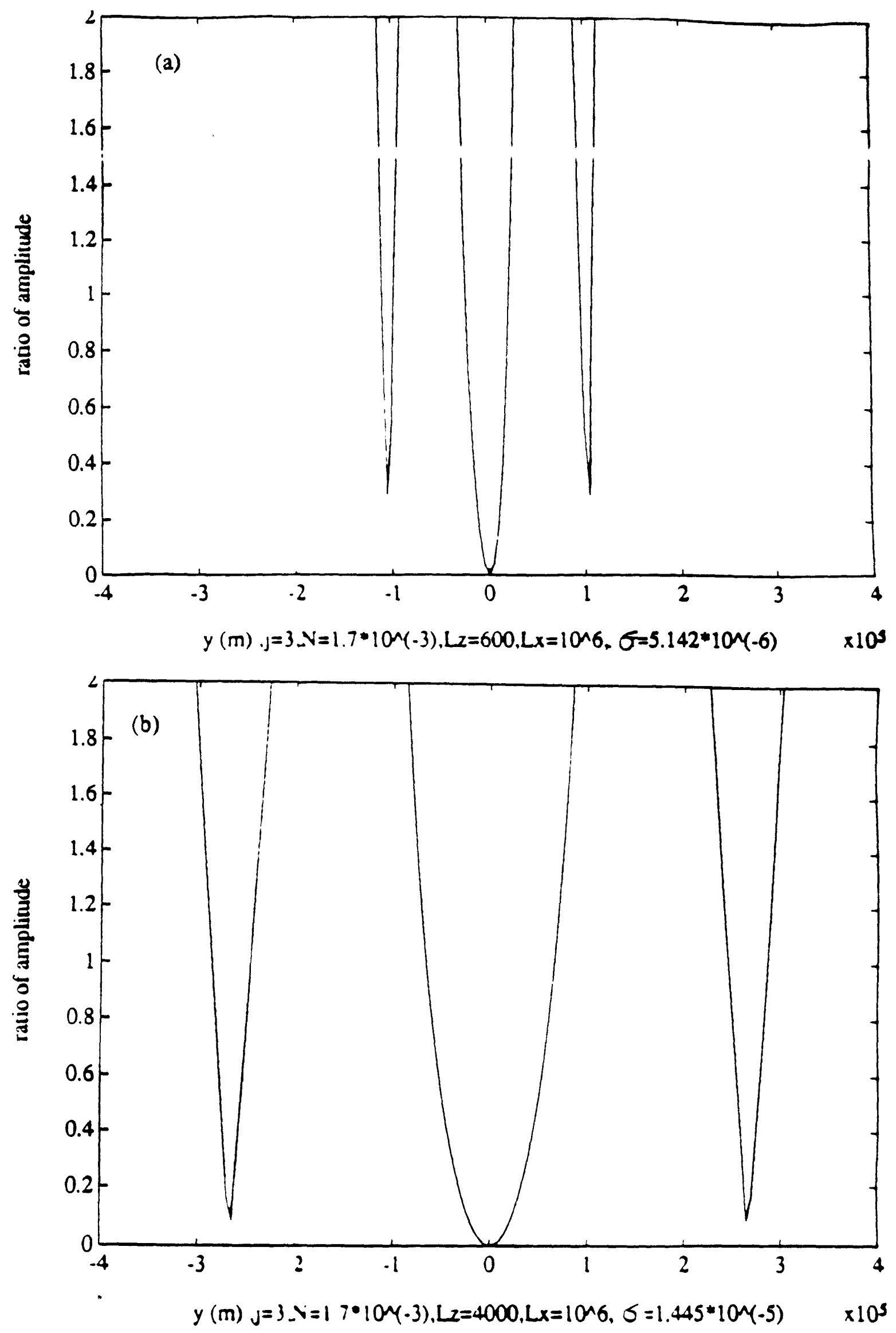

Fig. 7.12. Same as Fig.7.11 ercept for higher frequency waves. 


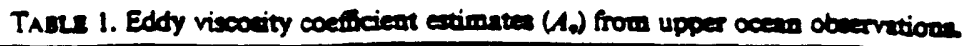

\begin{tabular}{|c|c|c|c|}
\hline Auther & $\left(10^{-3} \hat{m}^{A_{0}} \mathrm{~s}^{-1}\right)$ & Method & Location \\
\hline Hidale and Momoi (1961) & $1.0-2.0$ & $\begin{array}{l}\text { Ekman layer compared unth wiod stres } \\
\text { and surface curreat data }\end{array}$ & Tropical Pratie \\
\hline Jones (1973) & 3.9 & $\begin{array}{l}\text { Calculated A, as function of Richardsos } \\
\text { No. astumine a lopnthmic velocity } \\
\text { profile; used measurements of curreat } \\
\text { shear, temperature, and salinity. }\end{array}$ & Equatorial Pacific uodercurreat \\
\hline Smith (1974) & 40 & $\begin{array}{l}\text { High-resolutuon current meter } \\
\text { measurements of Reynolds surewes. }\end{array}$ & Arctic Oceas \\
\hline Halpern (1974) & $0.1-114$ & $\begin{array}{l}\text { Ekman layer theory compared with } \\
\text { moored mad and curreat } \\
\text { observauons }\end{array}$ & Nonbeastern Prabic \\
\hline Halpern (1976) & ss & Same as in Haldeen (1974). & Cosa of Orewa \\
\hline Halpern (1977) & 12.5 & $\begin{array}{l}\text { Balance betweea surface shear and wind } \\
\text { stress: moored mod and curreat data. }\end{array}$ & Northwea Africa \\
\hline Kase and Olbers (1979) & 30 & $\begin{array}{l}\text { Time-dependent Ekman model applied } \\
\text { to inersal waven }\end{array}$ & Athatic equatorial countercurrese \\
\hline Halpess (1980) & 96 & Same as in Halpero (1977). & Athatic equatorial countercurres \\
\hline Crawford and Osbom (1981) & 10 & $\begin{array}{l}\text { Dissupauon method: measured rurbulent } \\
\text { disupauon and undercurrent shear. }\end{array}$ & Central equatonal Pacate \\
\hline Greas ex al (1985) & $: 0$ & Same as in Crawford and Osbora (1981) & Central equatorial Prafic \\
\hline Peters et al. (1988) & 50 & Sarne as is Crawford and Osbors (1981) & Central equatorial Prabe \\
\hline McPlnaden ef al. (1988) & $10-180$ & $\begin{array}{l}\text { Time-dependeat Exman layer, moored } \\
\text { mnd and current dala }\end{array}$ & Wetern equntorisl Prabs \\
\hline
\end{tabular}

Table 1. Eddy viscosity coefficient estimates $\left(A_{v}\right)$ from upper ocean observations. (from Santiago-Mandujano and Finng, 1990) 